\title{
Positivity for Cluster Algebras of Rank 3
}

Dedicated to Robert Lazarsfeld on the occasion of his sixtieth birthday

\author{
by
}

Kyungyong LEE and Ralf SCHIFFLER

\begin{abstract}
We prove the positivity conjecture for skew-symmetric coefficient-free cluster algebras of rank 3.
\end{abstract}

2010 Mathematics Subject Classification: Primary 13F60.

Keywords: cluster algebra, positivity conjecture.

\section{$\S 1$. Introduction}

Cluster algebras have been introduced by Fomin and Zelevinsky in [13] in the context of total positivity and canonical bases in Lie theory. Since then cluster algebras have been shown to be related to various fields in mathematics including representation theory of finite-dimensional algebras, Teichmüller theory, Poisson geometry, combinatorics, Lie theory, tropical geometry and mathematical physics.

A cluster algebra is a subalgebra of a field of rational functions in $n$ variables $x_{1}, \ldots, x_{n}$, given by specifying a set of generators, the so-called cluster variables. These generators are constructed in a recursive way, starting from the initial variables $x_{1}, \ldots, x_{n}$, by a procedure called mutation, which is determined by the choice of a skew symmetric $n \times n$ integer matrix $B$ or, equivalently, by a quiver $Q$. Although each mutation is an elementary operation, it is very difficult to compute cluster variables in general, because of the recursive character of the construction.

Finding explicit computable direct formulas for the cluster variables is one of the main open problems in the theory of cluster algebras and has been studied

Communicated by H. Nakajima. Received August 28, 2012.

K. Lee: Department of Mathematics, Wayne State University, 656 West Kirby, Detroit, MI 48202, USA;

e-mail: klee@math.wayne.edu

R. Schiffler: Department of Mathematics, University of Connecticut,

196 Auditorium Road, U-3009, Storrs, CT 06269-3009, USA;

e-mail: schiffler@math.uconn.edu

(C) 2013 Research Institute for Mathematical Sciences, Kyoto University. All rights reserved. 
by many mathematicians. In 2002, Fomin and Zelevinsky showed that every cluster variable is a Laurent polynomial in the initial variables $x_{1}, \ldots, x_{n}$, and they conjectured that this Laurent polynomial has positive coefficients [13].

This positivity conjecture has been proved in the following special cases

- Acyclic cluster algebras. These are cluster algebras given by a quiver that is mutation equivalent to a quiver without oriented cycles. In this case, positivity has been shown in [17] building on [5, 16, 23, 24] using monoidal categorifications of quantum cluster algebras and perverse sheaves over graded quiver varieties. If the initial seed itself is acyclic, the conjecture has also been shown in [9] using Donaldson-Thomas theory.

- Cluster algebras from surfaces. In this case, positivity has been shown in [22] building on [27, 28, 26], using the fact that each cluster variable in such a cluster algebra corresponds to a curve in an oriented Riemann surface and the Laurent expansion of the cluster variable is determined by the crossing pattern of the curve with a fixed triangulation of the surface $[11,12]$. The construction and the proof of the positivity conjecture have been generalized to non-skew-symmetric cluster algebras from orbifolds in [10].

Our approach in this paper is different. We prove positivity almost exclusively by elementary algebraic computation. The advantage of this approach is that we do not need to restrict to a special type of cluster algebras but can work in the setting of an arbitrary cluster algebra. The drawback of our approach is that because of the sheer complexity of the computation, we need to restrict ourselves in this paper to rank three. Rank three is crucial since it is the smallest rank in which nonacyclic cluster algebras exist. Our main result is the following.

Theorem 1.1. The positivity conjecture holds in every skew-symmetric coefficientfree cluster algebra of rank 3 .

Our argument provides a method for the computation of the Laurent expansions of cluster variables, and we include some examples of explicit calculation. We point out that direct formulas for the Laurent polynomials have been obtained in several special cases. The most general results are the following:

- A formula involving the Euler-Poincaré characteristic of quiver Grassmannians obtained in [15, 8] using categorification and generalizing results in [6, 7]. While this formula shows a very interesting connection between cluster algebras and geometry, it is of limited computational use, since the Euler-Poincaré characteristics of quiver Grassmannians are hard to compute. In particular, this formula does not show positivity. On the other hand, the positivity result in this paper 
proves the positivity of the Euler-Poincaré characteristics of the quiver Grassmannians involved.

- An elementary combinatorial formula for cluster algebras from surfaces given in [22].

- A formula for cluster variables corresponding to string modules as a product of $2 \times 2$ matrices obtained in [1], generalizing a result in [2].

The main tools of the proof are modified versions of two formulas for the rank two case, one obtained by the first author in [18] and the other obtained by both authors in [20]. These formulas allow for the computation of the Laurent expansions of a given cluster variable with respect to any seed which is close enough to the variable in the sense that there is a sequence of mutations in only two vertices which links seed and variable. The general result then follows by inductive reasoning.

If the cluster algebra is not skew-symmetric, it is shown in $[25,19]$ that (an adaptation of) the second rank two formula still holds. We therefore expect that our argument can be generalized to prove the positivity conjecture for non-skewsymmetric cluster algebras of rank 3.

The article is organized as follows. We start by recalling some definitions and results from the theory of cluster algebras in Section 2. In Section 3, we present several formulas for the rank 2 case when considered inside a cluster algebra of rank 3. We use each of these formulas in the proof of the positivity conjecture for rank 3 in Section 4. An example is given in Section 5.

\section{$\S 2$. Cluster algebras}

In this section, we review some notions from the theory of cluster algebras.

\section{§2.1. Definition and Laurent phenomenon}

We begin by reviewing the definition of cluster algebra, first introduced by Fomin and Zelevinsky [13]. Our definition follows the exposition in [14].

To define a cluster algebra $\mathcal{A}$ we must first fix its ground ring. Let $(\mathbb{P}, \oplus, \cdot)$ be a semifield, i.e., an abelian multiplicative group endowed with a binary operation of (auxiliary) addition $\oplus$ which is commutative, associative, and distributive with respect to the multiplication in $\mathbb{P}$. The group ring $\mathbb{Z} \mathbb{P}$ will be used as a ground ring for $\mathcal{A}$.

As an ambient field for $\mathcal{A}$, we take a field $\mathcal{F}$ isomorphic to the field of rational functions in $n$ independent variables (here $n$ is the rank of $\mathcal{A}$ ), with coefficients in $\mathbb{Q P}$. Note that the definition of $\mathcal{F}$ does not involve the auxiliary addition in $\mathbb{P}$. 
Definition 2.1. A labeled seed in $\mathcal{F}$ is a triple $(\mathbf{x}, \mathbf{y}, B)$, where

- $\mathbf{x}=\left(x_{1}, \ldots, x_{n}\right)$ is an $n$-tuple from $\mathcal{F}$ forming a free generating set over $\mathbb{Q P}$,

- $\mathbf{y}=\left(y_{1}, \ldots, y_{n}\right)$ is an $n$-tuple from $\mathbb{P}$, and

- $B=\left(b_{i j}\right)$ is an $n \times n$ integer matrix which is skew-symmetrizable.

That is, $x_{1}, \ldots, x_{n}$ are algebraically independent over $\mathbb{Q P}$, and $\mathcal{F}=\mathbb{Q P}\left(x_{1}, \ldots, x_{n}\right)$. We refer to $\mathbf{x}$ as the (labeled) cluster of a labeled seed $(\mathbf{x}, \mathbf{y}, B)$, to the tuple $\mathbf{y}$ as the coefficient tuple, and to the matrix $B$ as the exchange matrix.

We use the notation $[x]_{+}=\max (x, 0),[1, n]=\{1, \ldots, n\}$, and

$$
\operatorname{sgn}(x)= \begin{cases}-1 & \text { if } x<0 \\ 0 & \text { if } x=0 \\ 1 & \text { if } x>0\end{cases}
$$

Definition 2.2. Let $(\mathbf{x}, \mathbf{y}, B)$ be a labeled seed in $\mathcal{F}$, and let $k \in[1, n]$. The seed mutation $\mu_{k}$ in direction $k$ transforms $(\mathbf{x}, \mathbf{y}, B)$ into the labeled seed $\mu_{k}(\mathbf{x}, \mathbf{y}, B)=$ $\left(\mathbf{x}^{\prime}, \mathbf{y}^{\prime}, B^{\prime}\right)$ defined as follows:

- The entries of $B^{\prime}=\left(b_{i j}^{\prime}\right)$ are given by

$$
b_{i j}^{\prime}= \begin{cases}-b_{i j} & \text { if } i=k \text { or } j=k, \\ b_{i j}+\operatorname{sgn}\left(b_{i k}\right)\left[b_{i k} b_{k j}\right]_{+} & \text {otherwise. }\end{cases}
$$

- The coefficient tuple $\mathbf{y}^{\prime}=\left(y_{1}^{\prime}, \ldots, y_{n}^{\prime}\right)$ is given by

$$
y_{j}^{\prime}= \begin{cases}y_{k}^{-1} & \text { if } j=k, \\ y_{j} y_{k}^{\left[b_{k j}\right]_{+}}\left(y_{k} \oplus 1\right)^{-b_{k j}} & \text { if } j \neq k .\end{cases}
$$

- The cluster $\mathbf{x}^{\prime}=\left(x_{1}^{\prime}, \ldots, x_{n}^{\prime}\right)$ is given by $x_{j}^{\prime}=x_{j}$ for $j \neq k$, whereas $x_{k}^{\prime} \in \mathcal{F}$ is determined by the exchange relation

$$
x_{k}^{\prime}=\frac{y_{k} \prod x_{i}^{\left[b_{i k}\right]_{+}}+\prod x_{i}^{\left[-b_{i k}\right]_{+}}}{\left(y_{k} \oplus 1\right) x_{k}} .
$$

We say that two exchange matrices $B$ and $B^{\prime}$ are mutation-equivalent if one can get from $B$ to $B^{\prime}$ by a sequence of mutations.

Definition 2.3. Consider the $n$-regular tree $\mathbb{T}_{n}$ whose edges are labeled by the numbers $1, \ldots, n$, so that the $n$ edges emanating from each vertex receive different labels. A cluster pattern is an assignment of a labeled seed $\Sigma_{t}=\left(\mathbf{x}_{t}, \mathbf{y}_{t}, B_{t}\right)$ to every vertex $t \in \mathbb{T}_{n}$, such that the seeds assigned to the endpoints of any edge $t \frac{k}{t} t^{\prime}$ are obtained from each other by the seed mutation in direction $k$. The components 
of $\Sigma_{t}$ are written as

$$
\mathbf{x}_{t}=\left(x_{1 ; t}, \ldots, x_{n ; t}\right), \quad \mathbf{y}_{t}=\left(y_{1 ; t}, \ldots, y_{n ; t}\right), \quad B_{t}=\left(b_{i j}^{t}\right) .
$$

Clearly, a cluster pattern is uniquely determined by an arbitrary seed.

Definition 2.4. Given a cluster pattern, we denote

$$
\mathcal{X}=\bigcup_{t \in \mathbb{T}_{n}} \mathbf{x}_{t}=\left\{x_{i, t}: t \in \mathbb{T}_{n}, 1 \leq i \leq n\right\},
$$

the union of clusters of all the seeds in the pattern. The elements $x_{i, t} \in \mathcal{X}$ are called cluster variables. The cluster algebra $\mathcal{A}$ associated with a given pattern is the $\mathbb{Z} \mathbb{P}$ subalgebra of the ambient field $\mathcal{F}$ generated by all cluster variables: $\mathcal{A}=\mathbb{Z} \mathbb{P}[\mathcal{X}]$. We denote $\mathcal{A}=\mathcal{A}(\mathbf{x}, \mathbf{y}, B)$, where $(\mathbf{x}, \mathbf{y}, B)$ is any seed in the underlying cluster pattern.

The cluster algebra is called skew-symmetric if the matrix $B$ is skew-symmetric. In this case, it is often convenient to represent the $n \times n$ matrix $B$ by a quiver $Q_{B}$ with vertices $1, \ldots, n$ and $\left[b_{i j}\right]_{+}$arrows from vertex $i$ to vertex $j$.

If $\mathbb{P}=1$ then the cluster algebra is said to be coefficient-free.

The main result in this paper is on coefficient-free cluster algebras. However, we need cluster algebras with coefficients in Section 3.

In [13], Fomin and Zelevinsky proved the remarkable Laurent phenomenon and posed the following positivity conjecture.

Theorem 2.5 (Laurent Phenomenon). For any cluster algebra $\mathcal{A}$ and any seed $\Sigma_{t}$, each cluster variable $x$ is a Laurent polynomial over $\mathbb{Z} \mathbb{P}$ in the cluster variables from $\mathbf{x}_{t}=\left(x_{1 ; t}, \ldots, x_{n ; t}\right)$.

Conjecture 2.6 (Positivity Conjecture). For any cluster algebra $\mathcal{A}$, any seed $\Sigma$, and any cluster variable $x$, the Laurent polynomial has coefficients which are nonnegative integer linear combinations of elements in $\mathbb{P}$.

\section{§2.2. Cluster algebras with principal coefficients}

One important choice for $\mathbb{P}$ is the tropical semifield; in this case we say that the corresponding cluster algebra is of geometric type.

Definition 2.7. Let $\operatorname{Trop}\left(u_{1}, \ldots, u_{m}\right)$ be an abelian group (written multiplicatively) freely generated by the $u_{j}$. We define $\oplus$ in $\operatorname{Trop}\left(u_{1}, \ldots, u_{m}\right)$ by

$$
\prod_{j} u_{j}^{a_{j}} \oplus \prod_{j} u_{j}^{b_{j}}=\prod_{j} u_{j}^{\min \left(a_{j}, b_{j}\right)}
$$

and call $\left(\operatorname{Trop}\left(u_{1}, \ldots, u_{m}\right), \oplus, \cdot\right)$ a tropical semifield. 
Remark 2.8. In cluster algebras whose ground ring is $\operatorname{Trop}\left(u_{1}, \ldots, u_{m}\right)$ (the tropical semifield), it is convenient to replace the matrix $B$ by an $(n+m) \times n$ matrix $\tilde{B}=\left(b_{i j}\right)$ whose upper part is the $n \times n$ matrix $B$ and whose lower part is an $m \times n$ matrix that encodes the coefficient tuple via

$$
y_{k}=\prod_{i=1}^{m} u_{i}^{b_{(n+i) k}} .
$$

Then the mutation of the coefficient tuple in (2) is determined by the mutation of the matrix $\tilde{B}$ in equation (1) and the formula (6); and the exchange relation (3) becomes

$$
x_{k}^{\prime}=x_{k}^{-1}\left(\prod_{i=1}^{n} x_{i}^{\left[b_{i k}\right]_{+}} \prod_{i=1}^{m} u_{i}^{\left[b_{(n+i) k}\right]_{+}}+\prod_{i=1}^{n} x_{i}^{\left[-b_{i k}\right]_{+}} \prod_{i=1}^{m} u_{i}^{\left[-b_{(n+i) k}\right]_{+}}\right) .
$$

Recall from [14] that a cluster algebra $\mathcal{A}$ is said to have principal coefficients at a vertex $t$ if $\mathbb{P}=\operatorname{Trop}\left(y_{1}, \ldots, y_{n}\right)$ and $\mathbf{y}_{t}=\left(y_{1}, \ldots, y_{n}\right)$.

Definition 2.9. Let $\mathcal{A}$ be the cluster algebra with principal coefficients at $t$, defined by the initial seed $\left(\mathbf{x}_{t}, \mathbf{y}_{t} B_{t}\right)$ with

$$
\mathbf{x}_{t}=\left(x_{1}, \ldots, x_{n}\right), \quad \mathbf{y}_{t}=\left(y_{1}, \ldots, y_{n}\right) .
$$

- Let $X_{\ell ; t}$ be the Laurent expansion of the cluster variable $x_{\ell ; t}$ in $x_{1}, \ldots, x_{n}$, $y_{1}, \ldots, y_{n}$.

- The F-polynomial of the cluster variable $x_{\ell, t}$ is defined as $F_{\ell ; t}=$ $X_{\ell ; t}\left(1, \ldots, 1 ; y_{1}, \ldots, y_{n}\right)$

- The $g$-vector $\mathbf{g}_{\ell ; t}$ of the cluster variable $x_{\ell, t}$ is defined as the degree vector of the monomial $X_{\ell ; t}\left(x_{1}, \ldots, x_{n} ; 0, \ldots, 0\right)$.

The following theorem shows that expansion formulas in principal coefficients can be used to compute expansions in arbitrary coefficient systems.

Theorem 2.10 ([14, Theorem 3.7]). Let $\mathcal{A}$ be a cluster algebra over an arbitrary semifield $\mathbb{P}$ with initial seed

$$
\left(\left(x_{1}, \ldots, x_{n}\right),\left(\hat{y}_{1}, \ldots, \hat{y}_{n}\right), B\right) .
$$

Then the cluster variables in $\mathcal{A}$ can be expressed as follows:

$$
x_{\ell ; t}=\frac{X_{\ell ; t}\left(x_{1}, \ldots, x_{n} ; \hat{y}_{1}, \ldots, \hat{y}_{n}\right)}{F_{\ell ; t} \mid \mathbb{P}\left(\hat{y}_{1}, \ldots, \hat{y}_{n}\right)} .
$$

where $F_{\ell ; t} \mid \mathbb{P}\left(\hat{y}_{1}, \ldots, \hat{y}_{n}\right)$ is the $F$-polynomial evaluated at $\hat{y}_{1}, \ldots, \hat{y}_{n}$ inside the semifield $\mathbb{P}$. 


\section{§3. Rank 2 considerations}

In this section, we use the rank 2 formula from [20] (in the parametrization of [19]) to compute in a nonacyclic cluster algebra of rank three the Laurent expansions of those cluster variables which are obtained from the initial cluster by a mutation sequence involving only two vertices.

\section{§3.1. Rank 2 formula}

We start by recalling from [19] the formula for the Laurent expansion of an arbitrary cluster variable in the cluster algebra of rank 2 given by the initial quiver with $r$ arrows

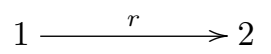

where $r \geq 2$ is a positive integer. The cluster variables $x_{n}$ in this cluster algebra are defined by the following recursion:

$$
x_{n+1}=\left(x_{n}^{r}+1\right) / x_{n-1} \quad \text { for any integer } n .
$$

Let $\left\{c_{n}^{[r]}\right\}_{n \in \mathbb{Z}}$ be the sequence defined by the recurrence relation

$$
c_{n}^{[r]}=r c_{n-1}^{[r]}-c_{n-2}^{[r]},
$$

with the initial condition $c_{1}^{[r]}=0, c_{2}^{[r]}=1$. The $c_{n}^{[r]}$ are Chebyshev polynomials. For example, if $r=2$ then $c_{n}^{[r]}=n-1$; if $r=3$, the sequence $c_{n}^{[r]}$ takes the following values:

$$
\ldots,-3,-1,0,1,3,8,21,55,144, \ldots
$$

The pair of the absolute values of the integers $\left(c_{n-1}^{[r]}, c_{n-2}^{[r]}\right)$ is the degree of the denominator of the cluster variable $x_{n}$.

If the value of $r$ is clear from the context, we usually write $c_{n}$ instead of $c_{n}^{[r]}$.

Lemma 3.1. Let $n \geq 3$. Then $c_{n-1} c_{n+k-3}-c_{n+k-2} c_{n-2}=c_{k}$ for $k \in \mathbb{Z}$. In particular, $c_{n-1}^{2}-c_{n} c_{n-2}=1$.

Proof. The result holds for $n=3$. Suppose that $n \geq 4$. Then

$$
\begin{aligned}
c_{n+k-2} c_{n-2} & =r c_{n+k-3} c_{n-2}-c_{n+k-4} c_{n-2} \stackrel{*}{=} r c_{n+k-3} c_{n-2}-\left(c_{k}+c_{n+k-3} c_{n-3}\right) \\
& =c_{n+k-3}\left(r c_{n-2}-c_{n-3}\right)-c_{k}=c_{n+k-3} c_{n-1}-c_{k},
\end{aligned}
$$

where $*$ holds by induction.

Let $\left(a_{1}, a_{2}\right)$ be a pair of nonnegative integers. A Dyck path of type $a_{1} \times a_{2}$ is a lattice path from $(0,0)$ to $\left(a_{1}, a_{2}\right)$ that never goes above the main diagonal joining $(0,0)$ and $\left(a_{1}, a_{2}\right)$. Among the Dyck paths of a given type $a_{1} \times a_{2}$, there 
is a (unique) maximal one denoted by $\mathcal{D}=\mathcal{D}^{a_{1} \times a_{2}}$. It is defined by the property that any lattice point strictly above $\mathcal{D}$ is also strictly above the main diagonal.

Let $\mathcal{D}=\mathcal{D}^{a_{1} \times a_{2}}$. Let $\mathcal{D}_{1}=\left\{u_{1}, \ldots, u_{a_{1}}\right\}$ be the set of horizontal edges of $\mathcal{D}$ indexed from left to right, and $\mathcal{D}_{2}=\left\{v_{1}, \ldots, v_{a_{2}}\right\}$ the set of vertical edges of $\mathcal{D}$ indexed from bottom to top. Given any points $A$ and $B$ on $\mathcal{D}$, let $A B$ be the subpath starting from $A$, and going in the northeast direction until it reaches $B$ (if we reach $\left(a_{1}, a_{2}\right)$ first, we continue from $\left.(0,0)\right)$. By convention, if $A=B$, then $A A$ is the subpath that starts from $A$, then passes $\left(a_{1}, a_{2}\right)$ and ends at $A$. If we represent a subpath of $\mathcal{D}$ by its set of edges, then for $A=(i, j)$ and $B=\left(i^{\prime}, j^{\prime}\right)$, we have

$$
A B= \begin{cases}\left\{u_{k}, v_{\ell}: i<k \leq i^{\prime}, j<\ell \leq j^{\prime}\right\} & \text { if } B \text { is to the northeast of } A \\ \mathcal{D}-\left\{u_{k}, v_{\ell}: i^{\prime}<k \leq i, j^{\prime}<\ell \leq j\right\} & \text { otherwise. }\end{cases}
$$

We denote by $(A B)_{1}$ the set of horizontal edges in $A B$, and by $(A B)_{2}$ the set of vertical edges in $A B$. Also let $A B^{\circ}$ denote the set of lattice points on the subpath $A B$ excluding the endpoints $A$ and $B$ (here $(0,0)$ and $\left(a_{1}, a_{2}\right)$ are regarded as the same point).

Here is an example for $\left(a_{1}, a_{2}\right)=(6,4)$.

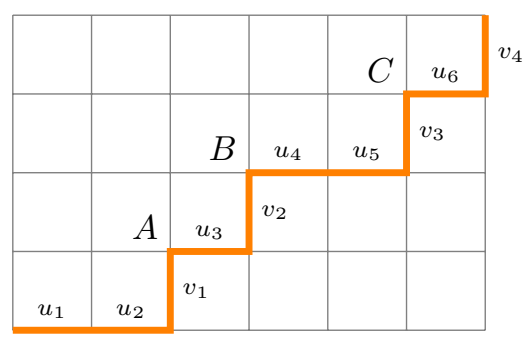

Figure 1. A maximal Dyck path.

Let $A=(2,1), B=(3,2)$ and $C=(5,3)$. Then

$(A B)_{1}=\left\{u_{3}\right\},(A B)_{2}=\left\{v_{2}\right\},(B A)_{1}=\left\{u_{4}, u_{5}, u_{6}, u_{1}, u_{2}\right\},(B A)_{2}=\left\{v_{3}, v_{4}, v_{1}\right\}$.

The point $C$ is in $B A^{\circ}$ but not in $A B^{\circ}$. The subpath $A A$ has length 10 (not 0 ).

Definition 3.2. For $S_{1} \subseteq \mathcal{D}_{1}, S_{2} \subseteq \mathcal{D}_{2}$, we say that the pair $\left(S_{1}, S_{2}\right)$ is compatible if for every $u \in S_{1}$ and $v \in S_{2}$, denoting by $E$ the left endpoint of $u$ and $F$ the upper endpoint of $v$, there exists a lattice point $A \in E F^{\circ}$ such that

$$
\left|(A F)_{1}\right|=r\left|(A F)_{2} \cap S_{2}\right| \quad \text { or } \quad\left|(E A)_{2}\right|=r\left|(E A)_{1} \cap S_{1}\right| .
$$


With all this terminology in place we are ready to present the combinatorial expression for greedy elements. The following has been proved in [21, 19].

Theorem 3.3. For $n \geq 3$, we have

$$
\begin{aligned}
x_{n} & =x_{1}^{-c_{n-1}} x_{2}^{-c_{n-2}} \sum_{\left(S_{1}, S_{2}\right)} x_{1}^{r\left|S_{2}\right|} x_{2}^{r\left|S_{1}\right|}, \\
x_{3-n} & =x_{2}^{-c_{n-1}} x_{1}^{-c_{n-2}} \sum_{\left(S_{1}, S_{2}\right)} x_{2}^{r\left|S_{2}\right|} x_{1}^{r\left|S_{1}\right|},
\end{aligned}
$$

where the sums are over all compatible pairs $\left(S_{1}, S_{2}\right)$ in $\mathcal{D}^{c_{n-1} \times c_{n-2}}$.

Remark 3.4. For $n=2$, the formula is consistent if we impose the additional convention that $\mathcal{D}^{c_{1} \times c_{0}}$ is the empty set.

Example 3.5. Let $r=3$ and $n=5$. Then $\mathcal{D}^{8 \times 3}$ is the following path:

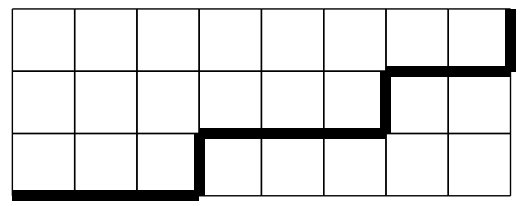

The illustrations below show the possible configurations for compatible pairs in $\mathcal{D}^{8 \times 3}$. If the edge $u_{i}$ is marked

- , then $u_{i}$ can occur in $S_{1}$.

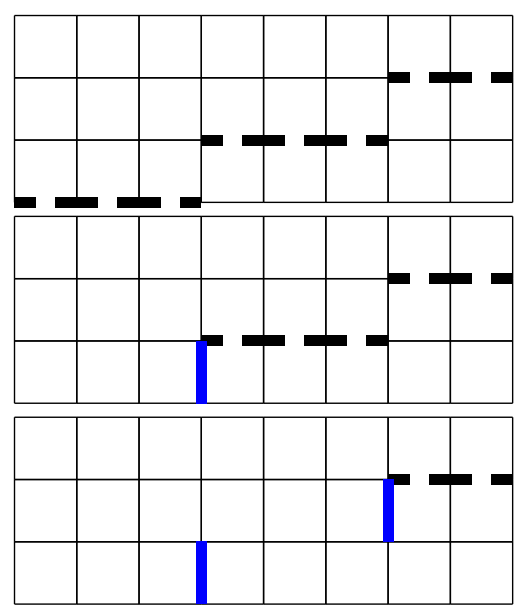

$\sum_{S_{1} \subset\left\{u_{1}, \ldots, u_{8}\right\}, S_{2}=\emptyset} x_{1}^{r\left|S_{2}\right|} x_{2}^{r\left|S_{1}\right|}$

$=\left(1+x_{2}^{3}\right)^{8}$

$\sum_{S_{1} \subset\left\{u_{4}, \ldots, u_{8}\right\}, S_{2}=\left\{v_{1}\right\}} x_{1}^{r\left|S_{2}\right|} x_{2}^{r\left|S_{1}\right|}$

$=x_{1}^{3}\left(1+x_{2}^{3}\right)^{5}$

$\sum_{S_{1} \subset\left\{u_{7}, u_{8}\right\}, S_{2}=\left\{v_{1}, v_{2}\right\}} x_{1}^{r\left|S_{2}\right|} x_{2}^{r\left|S_{1}\right|}$

$=x_{1}^{6}\left(1+x_{2}^{3}\right)^{2}$ 

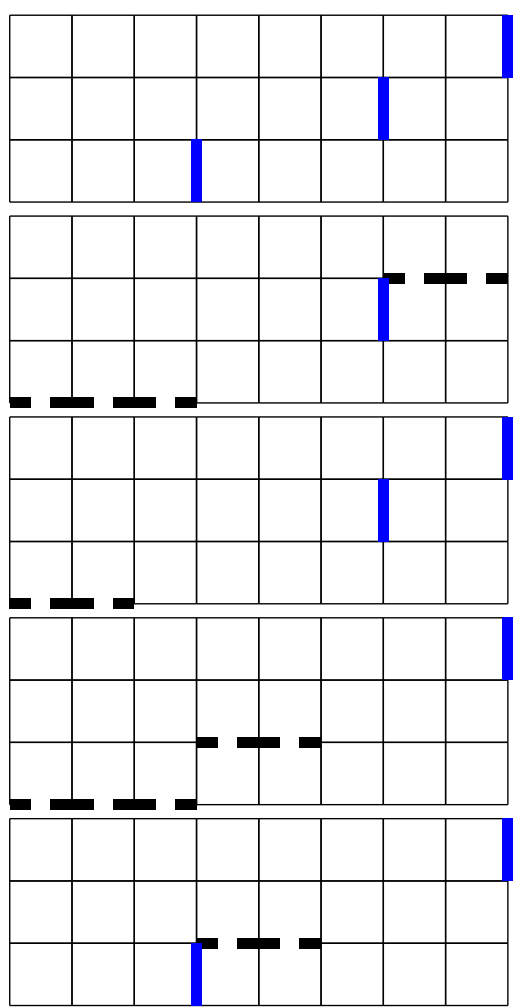

Adding the above eight polynomials together gives

$$
\begin{aligned}
x_{2}^{24}+8 x_{2}^{21}+3 x_{1}^{3} x_{2}^{15}+28 & x_{2}^{18}+15 x_{1}^{3} x_{2}^{12}+56 x_{2}^{15}+3 x_{1}^{6} x_{2}^{6} \\
& +30 x_{1}^{3} x_{2}^{9}+70 x_{2}^{12}+x_{1}^{9}+6 x_{1}^{6} x_{2}^{3}+30 x_{1}^{3} x_{2}^{6} \\
& +56 x_{2}^{9}+3 x_{1}^{6}+15 x_{1}^{3} x_{2}^{3}+28 x_{2}^{6}+3 x_{1}^{3}+8 x_{2}^{3}+1 .
\end{aligned}
$$

Then $x_{5}$ is obtained by dividing (12) by $x_{1}^{8} x_{2}^{3}$.

The following corollary can be adapted from results of [20]. Let $g_{\ell}$ be the $g$-vector and let $F_{\ell}$ be the $F$-polynomial of $x_{\ell}$, for all integers $\ell$. Then $g_{3}=$ $(-1, r), g_{0}=(0,-1), F_{3}=y_{1}+1$ and $F_{0}=y_{2}+1$, and all other cases are described in the following result.

Corollary 3.6. Let $n \geq 3$. Then

$$
\begin{array}{ll}
g_{n}=\left(-c_{n-1}, c_{n}\right), & g_{3-n}=\left(-c_{n-2}, c_{n-3}\right), \\
F_{n}=\sum_{\left(S_{1}, S_{2}\right)} y_{1}^{c_{n-1}-\left|S_{1}\right|} y_{2}^{\left|S_{2}\right|}, & F_{3-n}=\sum_{\left(S_{1}, S_{2}\right)} y_{1}^{c_{n-2}-\left|S_{2}\right|} y_{2}^{\left|S_{1}\right|},
\end{array}
$$

where the sum is over all compatible pairs $\left(S_{1}, S_{2}\right)$ in $\mathcal{D}^{c_{n-1} \times c_{n-2}}$. 
Recall from Definition 2.9 that $X_{n}$ is the Laurent polynomial in $x_{1}, x_{2}, y_{1}, y_{2}$ corresponding to the expansion of $x_{n}$ in the cluster algebra with principal coefficients in the seed $\left(\left(x_{1}, x_{2}\right),\left(y_{1}, y_{2}\right), Q\right)$.

Corollary 3.7. Let $n \geq 3$. Then

$$
X_{n}=x_{1}^{-c_{n-1}} x_{2}^{-c_{n-2}} \sum_{\left(S_{1}, S_{2}\right)} x_{1}^{r\left|S_{2}\right|} x_{2}^{r\left|S_{1}\right|} y_{1}^{c_{n-1}-\left|S_{1}\right|} y_{2}^{\left|S_{2}\right|} .
$$

Proof. This follows directly from Theorem 3.3 and Corollary 3.6.

\section{§3.2. A preliminary lemma}

Let $\mathcal{A}(Q)$ be a coefficient-free cluster algebra of rank 3 with initial quiver $Q$ equal to

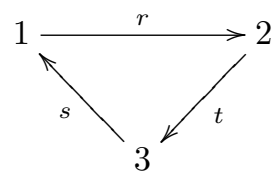

where $r, s, t$ denote the numbers of arrows.

Let $x_{n}$ be the cluster variable obtained from the seed $\left(\left(x_{1}, x_{2}, z_{3}\right), Q\right)$ by the sequence of $n-2$ mutations $1,2,1,2,1, \ldots$.

From Theorem 2.10, we have

$$
x_{n}=\frac{X_{n}\left(x_{1}, x_{2}, \hat{y}_{1}, \hat{y}_{2}\right)}{\left.F_{n}\right|_{\hat{\mathbb{P}}}\left(\hat{y}_{1}, \hat{y}_{2}\right)},
$$

where $X_{n}$ is as in Corollary 3.7, $\left(\hat{y}_{1}, \hat{y}_{2}\right)=\left(z_{3}^{s}, z_{3}^{-t}\right)$, and the denominator is the $F$-polynomial evaluated in $\hat{y}$ and with tropical addition. Thus, by Corollary 3.6, the denominator is $\sum_{\beta}^{\oplus} z_{3}^{s\left(c_{n-1}-\left|S_{1}\right|\right)-t\left|S_{2}\right|}$, which is equal to $z_{3}^{m}$ where $m$ is the smallest power occurring in this sum. Therefore we get

$$
x_{n}=x_{1}^{-c_{n-1}} x_{2}^{-c_{n-2}} \sum_{\left(S_{1}, S_{2}\right)} x_{1}^{r\left|S_{2}\right|} x_{2}^{r\left|S_{1}\right|} z_{3}^{s\left(c_{n-1}-\left|S_{1}\right|\right)-t\left|S_{2}\right|-m}
$$

where $m=\min _{\left(S_{1}, S_{2}\right)}\left(s\left(c_{n-1}-\left|S_{1}\right|\right)-t\left|S_{2}\right|\right)$.

We shall need a precise value for $m$. As a first step, we determine which compatible pair $\left(S_{1}, S_{2}\right)$ in $\mathcal{D}^{c_{n-1} \times c_{n-2}}$ can realize the minimum $m$.

Lemma 3.8. Let $s$ and $t$ be nonzero integers such that there are nonzero arrows between all pairs of the three vertices in any seed between the initial and terminal seeds inclusive. Then there is a unique compatible pair $\left(S_{1}, S_{2}\right)$ in $\mathcal{D}^{c_{n-1} \times c_{n-2}}$ which achieves $s\left(c_{n-1}-\left|S_{1}\right|\right)-t\left|S_{2}\right|=m$. Such an $\left(S_{1}, S_{2}\right)$ is either $\left(\mathcal{D}_{1}, \emptyset\right)$, $\left(\emptyset, \mathcal{D}_{2}\right)$, or $(\emptyset, \emptyset)$. Moreover, if $s$ and $t$ are positive, then $\left(S_{1}, S_{2}\right)$ is either $\left(\mathcal{D}_{1}, \emptyset\right)$ or $\left(\emptyset, \mathcal{D}_{2}\right)$. 
Proof. We use induction on $n$. Consider first the case $n=3$. Since $\mathcal{D}^{c_{2} \times c_{1}}=$ $\mathcal{D}^{1 \times 0}$, there are exactly two compatible pairs $\left(\emptyset, \mathcal{D}_{2}\right)=(\emptyset, \emptyset)$ and $\left(\mathcal{D}_{1}, \emptyset\right)$. Thus $s\left(c_{n-1}-\left|S_{1}\right|\right)-t\left|S_{2}\right|=-s\left|S_{1}\right|$ achieves its minimum at $\left(S_{1}, S_{2}\right)=\left(\mathcal{D}_{1}, \emptyset\right)$ if $s$ is positive, and at $\left(S_{1}, S_{2}\right)=\left(\emptyset, \mathcal{D}_{2}\right)$ if $s$ is negative.

Suppose now that $n \geq 3$. Consider the expression given in (13). By induction, there is a unique $\left(\bar{S}_{1}, \bar{S}_{2}\right)$ in $\mathcal{D}^{c_{n-1} \times c_{n-2}}$ such that $s\left(c_{n-1}-\left|S_{1}\right|\right)-t\left|S_{2}\right|=m$.

Suppose first that $\left(\bar{S}_{1}, \bar{S}_{2}\right)=\left(\mathcal{D}_{1}, \emptyset\right)$. Then the term in $(13)$ that is not divisible by $z_{3}$ is

$$
x_{1}^{-c_{n-1}} x_{2}^{-c_{n-2}} x_{2}^{r c_{n-1}}=x_{1}^{-c_{n-1}} x_{2}^{c_{n}} .
$$

If $t>0$ then $x_{n+1}$ is obtained from $x_{n}$ by substituting

$$
x_{1} \mapsto x_{2}, \quad x_{2} \mapsto \frac{x_{2}^{r}+z_{3}^{t}}{x_{1}} .
$$

So (14) becomes

$$
x_{2}^{-c_{n-1}}\left(\frac{x_{2}^{r}+z_{3}^{t}}{x_{1}}\right)^{c_{n}}=x_{1}^{-c_{n}} x_{2}^{-c_{n-1}} x_{1}^{0} x_{2}^{r c_{n}} z_{3}^{0}+\text { terms divisible by } z_{3} .
$$

Thus the term in the expression for $x_{n+1}$ that is not divisible by $z_{3}$ corresponds to a compatible pair $\left(S_{1}, S_{2}\right)$ in $\mathcal{D}^{c_{n} \times c_{n-1}}$ with $\left|S_{1}\right|=c_{n}$, so $\left(S_{1}, S_{2}\right)=\left(\mathcal{D}_{1}, \emptyset\right)$.

On the other hand, if $t<0$ then we substitute

$$
x_{1} \mapsto x_{2}, \quad x_{2} \mapsto \frac{x_{2}^{r} z_{3}^{-t}+1}{x_{1}},
$$

so (14) becomes

$$
x_{2}^{-c_{n-1}}\left(\frac{x_{2}^{r} z_{3}^{-t}+1}{x_{1}}\right)^{c_{n}}=x_{1}^{-c_{n}} x_{2}^{-c_{n-1}} x_{1}^{0} x_{2}^{0} z_{3}^{0}+\text { terms divisible by } z_{3} .
$$

Thus the term in the expression for $x_{n+1}$ that is not divisible by $z_{3}$ corresponds to a compatible pair $\left(S_{1}, S_{2}\right)$ in $\mathcal{D}^{c_{n} \times c_{n-1}}$ with $\left|S_{1}\right|=0$ and $\left|S_{2}\right|=0$, so $\left(S_{1}, S_{2}\right)$ $=(\emptyset, \emptyset)$.

Next suppose that $\left(\bar{S}_{1}, \bar{S}_{2}\right)=(\emptyset, \emptyset)$. Then $c_{n-1}-\left|\bar{S}_{1}\right|=c_{n-1},\left|\bar{S}_{2}\right|=0$, $m=s c_{n-1}$ and $t<0$ since $(\emptyset, \emptyset)$ realizes the minimum. Then

$$
\begin{aligned}
\sum_{\left(S_{1}, S_{2}\right):\left|S_{1}\right|=0} & x_{1}^{-c_{n-1}} x_{2}^{-c_{n-2}} x_{1}^{r\left|S_{2}\right|} x_{2}^{r\left|S_{1}\right|} z_{3}^{s\left(c_{n-1}-\left|S_{1}\right|\right)-t\left|S_{2}\right|-m} \\
& =\sum_{\left(S_{1}, S_{2}\right):\left|S_{1}\right|=0} x_{1}^{-c_{n-1}} x_{2}^{-c_{n-2}} x_{1}^{r\left|S_{2}\right|} z_{3}^{-t\left|S_{2}\right|}=x_{1}^{-c_{n-1}}\left(\frac{x_{1}^{r} z_{3}^{-t}+1}{x_{2}}\right)^{c_{n-2}},
\end{aligned}
$$

where the last identity holds because the condition $\left|S_{1}\right|=0$ means that every subset $S_{2}$ of $\left\{1, \ldots, c_{n-2}\right\}$ is compatible with $S_{1}$. 
Applying the map

$$
x_{1} \mapsto x_{2}, \quad x_{2} \mapsto \frac{x_{2}^{r} z_{3}^{-t}+1}{x_{1}}
$$

yields $x_{2}^{-c_{n-1}} x_{1}^{c_{n-2}}=x_{1}^{-c_{n}} x_{2}^{-c_{n-1}} x_{1}^{r c_{n-1}} x_{2}^{0}$, which corresponds to a compatible pair $\left(S_{1}, S_{2}\right)$ in $\mathcal{D}^{c_{n} \times c_{n-1}}$ with $\left|S_{1}\right|=0$ and $\left|S_{2}\right|=c_{n-1}$, thus $\left(S_{1}, S_{2}\right)=\left(\emptyset, \mathcal{D}_{2}\right)$.

Finally, if $\left(\bar{S}_{1}, \bar{S}_{2}\right)=\left(\emptyset, \mathcal{D}_{2}\right)$, then $\left|\bar{S}_{1}\right|=0,\left|\bar{S}_{2}\right|=c_{n-2}, m=s c_{n-1}-t c_{n-2}$ and $t$ must be positive since $\left(\emptyset, \mathcal{D}_{2}\right)$ realizes the minimum. Then

$$
\begin{aligned}
\sum_{\left(S_{1}, S_{2}\right):\left|S_{1}\right|=0} & x_{1}^{-c_{n-1}} x_{2}^{-c_{n-2}} x_{1}^{r\left|S_{2}\right|} x_{2}^{r\left|S_{1}\right|} z_{3}^{s\left(c_{n-1}-\left|S_{1}\right|\right)-t\left|S_{2}\right|-m} \\
& =\sum_{\left(S_{1}, S_{2}\right):\left|S_{1}\right|=0} x_{1}^{-c_{n-1}} x_{2}^{-c_{n-2}} x_{1}^{r\left|S_{2}\right|} z_{3}^{t c_{n-2}-t\left|S_{2}\right|}=x_{1}^{-c_{n-1}}\left(\frac{x_{1}^{r}+z_{3}^{t}}{x_{2}}\right)^{c_{n-2}}
\end{aligned}
$$

and applying the map

$$
x_{1} \mapsto x_{2}, \quad x_{2} \mapsto \frac{x_{2}^{r}+z_{3}^{t}}{x_{1}}
$$

yields $x_{2}^{-c_{n-1}} x_{1}^{c_{n-2}}=x_{1}^{-c_{n}} x_{2}^{-c_{n-1}} x_{1}^{r c_{n-1}} x_{2}^{0}$, which corresponds to a compatible pair $\left(S_{1}, S_{2}\right)$ in $\mathcal{D}^{c_{n} \times c_{n-1}}$ with $\left|S_{1}\right|=0$ and $\left|S_{2}\right|=c_{n-1}$, thus $\left(S_{1}, S_{2}\right)=\left(\emptyset, \mathcal{D}_{2}\right)$.

\section{§3.3. Rank 2 inside rank 3: Dyck path formula}

Let $\mathcal{A}(Q)$ be a nonacyclic coefficient-free cluster algebra of rank 3 with initial quiver $Q$ equal to

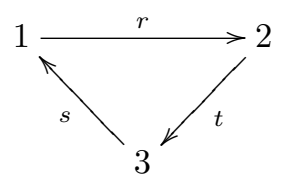

where $r, s, t$ denote the numbers of arrows. Now suppose that $r, s$ and $t$ are such that the cluster algebra $\mathcal{A}(Q)$ is nonacyclic. Then we can show that $m$ is always zero.

Corollary 3.9. If the cluster algebra is nonacyclic then there is a unique compatible pair $\left(S_{1}, S_{2}\right)$ in $\mathcal{D}^{c_{n-1} \times c_{n-2}}$, which achieves $s\left(c_{n-1}-\left|S_{1}\right|\right)-t\left|S_{2}\right|=m$. Moreover $\left(S_{1}, S_{2}\right)=\left(\mathcal{D}_{1}, \emptyset\right)$ and $m=0$.

Proof. We use induction on $n$. For $n=3$ and $n=4$, computing $x_{n}$ directly by mutation yields

$$
x_{3}=\left(x_{2}^{r}+z_{3}^{s}\right) / x_{1}, \quad x_{4}=\left(x_{3}^{r}+z_{3}^{r s-t}\right) / x_{2},
$$

and hence the term in the expression for $x_{4}$ that is not divisible by $z_{3}$ is $x_{2}^{r^{2}} x_{1}^{-r} x_{2}^{-1}$, which corresponds to a compatible pair $\left(S_{1}, S_{2}\right)$ in $\mathcal{D}^{c_{n-1} \times c_{n-2}}$ such that $\left|S_{2}\right|=$ $c_{n-1}-\left|S_{1}\right|=0$, thus $\left(S_{1}, S_{2}\right)=\left(\mathcal{D}_{1}, \emptyset\right)$. 
Now the result follows by the same argument as in the proof of Lemma 3.8 using the fact that $t>0$.

We have proved the following theorem.

Theorem 3.10. For $n \geq 3$,

$$
x_{n}=x_{1}^{-c_{n-1}} x_{2}^{-c_{n-2}} \sum_{\left(S_{1}, S_{2}\right)} x_{1}^{r\left|S_{2}\right|} x_{2}^{r\left|S_{1}\right|} z_{3}^{s\left(c_{n-1}-\left|S_{1}\right|\right)-t\left|S_{2}\right|},
$$

where the sum is over all compatible pairs $\left(S_{1}, S_{2}\right)$ in $\mathcal{D}^{c_{n-1} \times c_{n-2}}$.

Remark 3.11. It follows from results of [25] and [19] that an adapted version of this theorem still holds if the cluster algebra is not skew-symmetric.

Our next goal is to describe cluster monomials of the form $x_{n+1}^{p} x_{n}^{q}$ with $p, q \geq$ 0 . In order to simplify the notation we define $A_{i}=p c_{i+1}+q c_{i}$. The following lemma is a straightforward consequence of Lemma 3.1 .

Lemma 3.12. For any $i$, we have

(a) $A_{i}=r A_{i-1}-A_{i-2}$,

(b) $A_{i}^{2}-A_{i+1} A_{i-1}=p^{2}+q^{2}+r p q$.

Theorem 3.13.

$$
x_{n+1}^{p} x_{n}^{q}=x_{1}^{-A_{n-1}} x_{2}^{-A_{n-2}} \sum_{\left(S_{1}, S_{2}\right)} x_{1}^{r\left|S_{2}\right|} x_{2}^{r\left|S_{1}\right|} z_{3}^{s\left(c_{n-1}-\left|S_{1}\right|\right)-t\left|S_{2}\right|},
$$

where the sum is over all $\left(S_{1}=\bigcup_{i=1}^{p+q} S_{1}^{i}, S_{2}=\bigcup_{i=1}^{p+q} S_{2}^{i}\right)$ such that

$$
\left(S_{1}^{i}, S_{2}^{i}\right) \text { is a compatible pair in } \begin{cases}\mathcal{D}^{c_{n-1} \times c_{n-2}} & \text { if } 1 \leq i \leq q, \\ \mathcal{D}^{c_{n} \times c_{n-1}} & \text { if } q+1 \leq i \leq p+q .\end{cases}
$$

Proof. This follows immediately from Theorem 3.10 .

Remark 3.14. It can be shown that the summation on the right hand side in Theorem 3.13 can be taken over all compatible pairs in $\mathcal{D}^{A_{n-1} \times A_{n-2}}$ instead, without changing the sum (see [19, Theorem 1.11]).

\section{§3.4. Rank 2 inside rank 3: Mixed formula}

We keep the setup of the previous subsection. In particular, $\mathcal{A}(Q)$ is nonacyclic. We present another formula for the Laurent expansion of the cluster monomial $x_{n+1}^{p} x_{n}^{q}$, which is parametrized by a certain sequence of integers $\tau_{0}, \tau_{1}, \ldots, \tau_{n-2}$. This formula is a generalization of a formula given in [18, Theorem 2.1]. Com- 
bining it with the formula of Theorem 3.13 yields the mixed formula of Theorem 3.21 below, which is a key ingredient for the proof of the positivity conjecture in Section 4.

For arbitrary (possibly negative) integers $A, B$, we define the modified binomial coefficient as follows:

$$
\left[\begin{array}{l}
A \\
B
\end{array}\right]:= \begin{cases}\prod_{i=0}^{A-B-1} \frac{A-i}{A-B-i} & \text { if } A>B \\
1 & \text { if } A=B \\
0 & \text { if } A<B\end{cases}
$$

If $A \geq 0$ then $\left[\begin{array}{c}c A \\ B\end{array}\right]=\left[\begin{array}{c}c A \\ A-B\end{array}\right]$ is just the usual binomial coefficient. In particular $\left[\begin{array}{c}c A \\ B\end{array}\right]=0$ if $A \geq 0$ and $B<0$.

For a sequence $\left(\tau_{j}\right)$ (respectively $\left(\tau_{j}^{\prime}\right)$ ) of integers, we define a sequence $\left(s_{i}\right)$ (respectively $\left(s_{i}^{\prime}\right)$ ) of weighted partial sums as follows:

$$
\begin{aligned}
& s_{0}=0, \quad s_{i}=\sum_{j=0}^{i-1} c_{i-j+1} \tau_{j}=c_{i+1} \tau_{0}+c_{i} \tau_{1}+\cdots+c_{2} \tau_{i-1}, \\
& s_{0}^{\prime}=0, \quad s_{i}^{\prime}=\sum_{j=0}^{i-1} c_{i-j+1} \tau_{j}^{\prime}=c_{i+1} \tau_{0}^{\prime}+c_{i} \tau_{1}^{\prime}+\cdots+c_{2} \tau_{i-1}^{\prime} .
\end{aligned}
$$

For example, $s_{1}=c_{2} \tau_{0}=\tau_{0}, s_{2}=c_{3} \tau_{0}+c_{2} \tau_{1}=r \tau_{0}+\tau_{1}$.

Lemma 3.15. $s_{n}=r s_{n-1}-s_{n-2}+\tau_{n-1}$.

Proof. Since $c_{n-j+1}=r c_{n-j}-c_{n-j-1}$, we see that $s_{n}$ is equal to

$$
\begin{aligned}
& \sum_{j=0}^{n-1}\left(r c_{n-j}-c_{n-j-1}\right) \tau_{j} \\
& \quad=r\left(\sum_{j=0}^{n-2} c_{n-j} \tau_{j}\right)+r c_{1} \tau_{n-1}-\left(\sum_{j=0}^{n-3} c_{n-j-1} \tau_{j}\right)-c_{1} \tau_{n-2}-c_{0} \tau_{n-1} \\
& =r s_{n-1}-s_{n-2}+\tau_{n-1},
\end{aligned}
$$

where the last identity holds because $c_{1}=0, c_{0}=-1$.

Definition 3.16. Let $\mathcal{L}\left(\tau_{0}, \tau_{1}, \ldots, \tau_{n-2}\right)$ denote the set of all $\left(\tau_{0}^{\prime}, \tau_{1}^{\prime}, \ldots, \tau_{n-2}^{\prime}\right)$ $\in \mathbb{Z}^{n-1}$ satisfying the conditions

- $0 \leq \tau_{i}^{\prime} \leq \tau_{i}$ for $0 \leq i \leq n-3$,

- $s_{n-2}^{\prime}=k c_{n-1}$ and $s_{n-1}^{\prime}=k c_{n}$ for some integer $0 \leq k \leq p$.

We define a partial order on $\mathcal{L}\left(\tau_{0}, \tau_{1}, \ldots, \tau_{n-2}\right)$ by

$\left(\tau_{0}^{\prime}, \tau_{1}^{\prime}, \ldots, \tau_{n-2}^{\prime}\right) \leq_{\mathcal{L}}\left(\tau_{0}^{\prime \prime}, \tau_{1}^{\prime \prime}, \ldots, \tau_{n-2}^{\prime \prime}\right) \quad$ if and only if $\tau_{i}^{\prime} \leq \tau_{i}^{\prime \prime}$ for $0 \leq i \leq n-3$. 
Let $\mathcal{L}_{\max }\left(\tau_{0}, \tau_{1}, \ldots, \tau_{n-2}\right)$ be the set of maximal elements of $\mathcal{L}\left(\tau_{0}, \tau_{1}, \ldots, \tau_{n-2}\right)$ with respect to $\leq_{\mathcal{L}}$.

We are ready to state the main result of this subsection.

Theorem 3.17. Let $n \geq 3$. Then

$$
\begin{aligned}
& x_{n+1}^{p} x_{n}^{q}= \\
& x_{1}^{-A_{n-1}} x_{2}^{-A_{n-2}} \sum_{\tau_{0}, \tau_{1}, \ldots, \tau_{n-2}}\left(\prod_{i=0}^{n-2}\left[\begin{array}{c}
A_{i+1}-r s_{i} \\
\tau_{i}
\end{array}\right]\right) x_{1}^{r s_{n-2}} x_{2}^{r\left(A_{n-1}-s_{n-1}\right)} z_{3}^{s s_{n-1}-t s_{n-2}} \text {, }
\end{aligned}
$$

where the summation runs over all integers $\tau_{0}, \ldots, \tau_{n-2}$ satisfying

$$
\left\{\begin{array}{l}
0 \leq \tau_{i} \leq A_{i+1}-r s_{i}(0 \leq i \leq n-3) \\
\tau_{n-2} \leq A_{n-1}-r s_{n-2}, \\
\left(s_{n-1}-s_{n-1}^{\prime}\right) A_{n-2} \geq\left(s_{n-2}-s_{n-2}^{\prime}\right) A_{n-1} \\
\quad \text { for any }\left(\tau_{0}^{\prime}, \ldots, \tau_{n-2}^{\prime}\right) \in \mathcal{L}_{\max }\left(\tau_{0}, \ldots, \tau_{n-2}\right) .
\end{array}\right.
$$

Proof. The theorem is proved in Section 3.5.

Example 3.18. Let $Q$ be the quiver

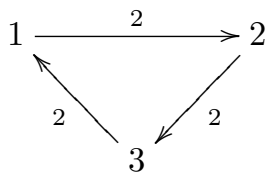

and let $n=5, p=1, q=0$. Thus our formula computes the cluster variable obtained from the initial cluster by mutating in directions $1,2,1$ and 2 .

First note that in this case $c_{i}=i-1, A_{i}=i$ and $s_{i}=i \tau_{0}+(i-1) \tau_{1}+\cdots+$ $2 \tau_{i-2}+\tau_{i-1}$. The first condition in (16) is $0 \leq \tau_{i} \leq i+1-2 s_{i}$. From this we see that $\tau_{0}$ is either 0 or 1 . If $\tau_{0}=1$, then $s_{1}=1$, hence $\tau_{1}=0$ by $(16)$, whence $s_{2}=2$ and $0 \leq \tau_{2} \leq(2+1)-2(2)$, again by (16), a contradiction. Thus $\tau_{0}=0$ and the conditions on $\tau_{i}$ in (16) become

$$
\tau_{0}=0 \quad 0 \leq \tau_{1} \leq 2 \quad 0 \leq \tau_{2} \leq 3-2 \tau_{1} \quad \tau_{3} \leq 4-4 \tau_{1}-2 \tau_{2},
$$

From this we conclude that there are the following 11 possibilities for $\left(\tau_{0}, \tau_{1}, \tau_{2}, \tau_{3}\right)$ :

$$
\begin{array}{cccccc}
(0,0,0,0) & (0,0,0,1) & (0,0,0,2) & (0,0,0,3) & (0,0,0,4) & (0,0,1,0) \\
& (0,0,1,1) & (0,0,1,2) & (0,0,2,0) & (0,0,3,-2) & (0,1,0,0)
\end{array}
$$

Observe that each of these tuples satisfies the second condition in (16). Indeed, the integer $k \in\{0,1\}$ in Definition 3.16 must satisfy

$$
3 k=2 \tau_{1}^{\prime}+\tau_{2}^{\prime} \quad \text { and } \quad 4 k=3 \tau_{1}^{\prime}+2 \tau_{2}^{\prime}+\tau_{3}^{\prime},
$$


so for example, if $\left(\tau_{0}, \tau_{1}, \tau_{2}, \tau_{3}\right)=(0,0,1,1)$ then $\tau_{0}^{\prime}=\tau_{1}^{\prime}=0,0 \leq \tau_{2}^{\prime} \leq 1, \tau_{3}^{\prime} \leq 1$ and

$$
3 k=\tau_{2}^{\prime} \leq 1 \quad \text { and } \quad 4 k=2 \tau_{2}^{\prime}+\tau_{3}^{\prime} .
$$

Thus $k=0, \mathcal{L}(0,0,1,1)=\{(0,0,0,0)\}$, and the second condition in (16) becomes

$$
\left(s_{4}-s_{4}^{\prime}\right) A_{3} \geq\left(s_{3}-s_{3}^{\prime}\right) A_{4} \Leftrightarrow(2+1-0) 3 \geq(1-0) 4 \Leftrightarrow 9 \geq 4 .
$$

On the other hand, the eleven 4 -tuples above are the only ones that satisfy all conditions in (16). For example, for the tuple $(0,1,1,-2)$, we get $k=1, \tau^{\prime}=$ $(0,1,1,-1) \in \mathcal{L}_{\max }(0,1,1,-2)$ and the condition

$\left(s_{4}-s_{4}^{\prime}\right) A_{3} \geq\left(s_{3}-s_{3}^{\prime}\right) A_{4} \Leftrightarrow(3+2-2-3-2+1) 3 \geq(2+1-2-1) 4 \Leftrightarrow-3 \geq 0$

is not satisfied. Therefore Theorem 3.17 yields

$$
\begin{aligned}
x_{6}=\left(x_{2}^{8}\right. & +4 x_{2}^{6} z_{3}^{2}+6 x_{2}^{4} z_{3}^{4}+4 x_{2}^{2} z_{3}^{6}+z_{3}^{8}+3 x_{1}^{2} x_{2}^{4} z_{3}^{2} \\
& \left.+6 x_{1}^{2} x_{2}^{2} z_{3}^{4}+3 x_{1}^{2} z_{3}^{6}+3 x_{1}^{4} z_{3}^{4}+x_{1}^{6} z_{3}^{2}+2 x_{1}^{4} x_{2}^{2} z_{3}^{2}\right) / x_{1}^{4} x_{2}^{3} .
\end{aligned}
$$

Remark 3.19. When comparing the formula of Theorem 3.17 with the Dyck path formula of Theorem 3.13, we have the following interpretation for the integer $k$ in Definition 3.16. Let $\mathcal{D}_{2}$ be the set of all vertical edges in $\mathcal{D}^{c_{n} \times c_{n-1}}$, and fix a pair $\left(S_{1}, S_{2}\right)$ as in Theorem 3.13. Then $k$ in Definition 3.15 is equal to the number of times $\mathcal{D}_{2}$ appears in $S_{2}$. Moreover, if $\left(\tau_{0}^{\prime}, \tau_{1}^{\prime}, \ldots, \tau_{n-2}^{\prime}\right) \in \mathcal{L}_{\max }$ is such that $\prod_{i=0}^{n-2}\left[\underset{\tau_{i}}{c A_{i+1}-r s_{i}}\right] \neq 0$ then

$$
k=\min \left(\left\lfloor\frac{s_{n-2}}{c_{n-1}}\right\rfloor, p\right) .
$$

Corollary 3.20. Let $x_{3}=\left(x_{2}^{r}+z_{3}^{s}\right) / x_{1}$ and let $t^{\prime}=s$ and $s^{\prime}=r s-t$ be the numbers of arrows from 1 to 3 and from 3 to 2 respectively, in the quiver obtained from $Q$ by mutating at vertex 1 . Then

$$
x_{2}^{-A_{n-2}} x_{3}^{-A_{n-3}} \sum_{\tau_{0}, \tau_{1}, \ldots, \tau_{n-3}}\left(\prod_{i=0}^{n-3}\left[\begin{array}{c}
A_{i+1}-r s_{i} \\
\tau_{i}
\end{array}\right]\right) x_{2}^{r s_{n-3}} x_{3}^{r\left(A_{n-2}-s_{n-2}\right)} z_{3}^{s^{\prime} s_{n-2}-t^{\prime} s_{n-3}},
$$

where the summation runs over all integers $\tau_{0}, \ldots, \tau_{n-3}$ satisfying

$$
\left\{\begin{array}{r}
0 \leq \tau_{i} \leq A_{i+1}-r s_{i}(0 \leq i \leq n-4), \tau_{n-3} \leq A_{n-2}-r s_{n-3} \\
\left(s_{n-2}-s_{n-2}^{\prime}\right) A_{n-3} \geq\left(s_{n-3}-s_{n-3}^{\prime}\right) A_{n-2} \\
\quad \text { for any }\left(\tau_{0}^{\prime}, \ldots, \tau_{n-3}^{\prime}\right) \in \mathcal{L}_{\max }\left(\tau_{0}, \ldots, \tau_{n-3}\right) .
\end{array}\right.
$$

Proof. This follows directly from Theorem 3.17. 
Combining Theorem 3.17 with Theorem 3.13 we get the following mixed formula.

Theorem 3.21. Let $n \geq 3$. Then

$$
\begin{aligned}
& x_{n+1}^{p} x_{n}^{q} \\
= & \sum_{\substack{\tau_{0}, \tau_{1}, \ldots, \tau_{n-3} \\
s_{n-2} \leq A_{n-1} / r}}\left(\prod_{i=0}^{n-3}\left(\begin{array}{c}
A_{i+1}-r s_{i} \\
\tau_{i}
\end{array}\right)\right) x_{3}^{A_{n-1}-r s_{n-2}} x_{2}^{r s_{n-3}-A_{n-2}} z_{3}^{s^{\prime} s_{n-2}-t^{\prime} s_{n-3}} \\
& +\sum_{\substack{\left(S_{1}, S_{2}\right) \\
r\left|S_{2}\right|-A_{n-1}>0}} x_{1}^{r\left|S_{2}\right|-A_{n-1}} x_{2}^{r\left|S_{1}\right|-A_{n-2}} z_{3}^{s\left(c_{n-1}-\left|S_{1}\right|\right)-t\left|S_{2}\right|} .
\end{aligned}
$$

Remark 3.22. The exponents of $x_{3}, x_{1}$ and $z_{3}$ are nonnegative, which is important for the proof of Theorem 4.3. The modified binomial coefficients can be replaced by the usual binomial coefficients, because the condition $s_{n-2} \leq A_{n-1} / r$ implies that $A_{n-2}-r s_{n-3}$ is nonnegative (see Lemma 3.24 below).

Proof. The first sum of the statement is obtained from Corollary 3.20 using Lemma 3.12 (a) for the exponent of $x_{3}$. Observe that the new condition $s_{n-2} \leq A_{n-1} / r$ in the summation precisely means that the exponent of $x_{3}$ is nonnegative. On the other hand, the sum of all terms in which the exponent of $x_{3}$ is negative in the expression in Corollary 3.20 is equal to the second sum in the statement of Theorem 3.21. This follows from the formula of Theorem 3.13.

In [4] the upper cluster algebra was defined as the intersection of the rings of Laurent polynomials in the $n+1$ clusters consisting of the initial cluster and all clusters obtained from it by a single mutation. The following corollary gives a different "upper bound" for the cluster monomials in the rank 2 direction. This new upper bound is defined as the semi-ring of polynomials in the variables in the initial cluster, the first mutation of one initial variable and the inverse of another initial cluster variable.

Corollary 3.23. Let $\widetilde{x}_{1}$ denote the cluster variable obtained from the initial seed by mutating in $x_{1}$. Then

$$
x_{n+1}^{p} x_{n}^{q} \in \mathbb{Z}_{\geq 0}\left[x_{1}, \widetilde{x}_{1}, z_{3}, x_{2}^{ \pm 1}\right] .
$$

Proof. This follows from Theorem 3.21 because $\widetilde{x}_{1}=x_{3}$.

\section{§3.5. Proof of Theorem 3.17}

We use induction on $n$. Suppose first that $n=3$. Since $x_{3}=\left(x_{2}^{r}+z_{3}^{s}\right) / x_{1}$, we have

$$
x_{3}^{a}=x_{1}^{-a} \sum_{\tau_{1}=0}^{a}\left(\begin{array}{c}
a \\
\tau_{1}
\end{array}\right) x_{2}^{r\left(a-\tau_{1}\right)} z_{3}^{s \tau_{1}},
$$


and since $x_{4}=\left(x_{3}^{r}+z_{3}^{r s-t}\right) / x_{2}$, we have

$$
x_{4}^{p}=x_{2}^{-p} \sum_{\tau_{0}=0}^{p}\left(\begin{array}{c}
p \\
\tau_{0}
\end{array}\right) x_{3}^{r\left(p-\tau_{0}\right)} z_{3}^{(r s-t) \tau_{0}} .
$$

Therefore

$$
\begin{aligned}
& x_{4}^{p} x_{3}^{q}=x_{2}^{-p} \sum_{\tau_{0}=0}^{p}\left(\begin{array}{c}
p \\
\tau_{0}
\end{array}\right) x_{3}^{r\left(p-\tau_{0}\right)+q} z_{3}^{(r s-t) \tau_{0}} \\
& =x_{2}^{-p} \sum_{\tau_{0}=0}^{p}\left(\begin{array}{c}
p \\
\tau_{0}
\end{array}\right) \sum_{\tau_{1}=0}^{r\left(p-\tau_{0}\right)+q}\left(\begin{array}{c}
r\left(p-\tau_{0}\right)+q \\
\tau_{1}
\end{array}\right) x_{1}^{-r\left(p-\tau_{0}\right)-q} x_{2}^{r\left(r\left(p-\tau_{0}\right)+q-\tau_{1}\right)} z_{3}^{s \tau_{1}} z_{3}^{(r s-t) \tau_{0}} \\
& =x_{1}^{-r p-q} x_{2}^{-p} \sum_{\tau_{0}=0}^{p} \sum_{\tau_{1}=0}^{r\left(p-\tau_{0}\right)+q}\left(\begin{array}{c}
p \\
\tau_{0}
\end{array}\right)\left(\begin{array}{c}
r\left(p-\tau_{0}\right)+q \\
\tau_{1}
\end{array}\right) x_{1}^{r \tau_{0}} x_{2}^{r\left(r\left(p-\tau_{0}\right)+q-\tau_{1}\right)} z_{3}^{s \tau_{1}+(r s-t) \tau_{0}},
\end{aligned}
$$

and the statement follows from $A_{1}=p, A_{2}=r p+q, s_{0}=0, s_{1}=\tau_{0}, s_{2}=r \tau_{0}+\tau_{1}$.

Suppose now that $n \geq 4$, and assume that the statement holds for $n$ or less.

Then by the obvious shift, we have

$$
\begin{aligned}
& x_{n+2}^{p} x_{n+1}^{q} \\
& \quad=x_{2}^{-A_{n-1}} x_{3}^{-A_{n-2}} \sum_{\tau_{0}, \tau_{1}, \ldots, \tau_{n-2}}\left[\left(\prod_{i=0}^{n-2}\left[\begin{array}{c}
A_{i+1}-r s_{i} \\
\tau_{i}
\end{array}\right]\right) x_{2}^{r s_{n-2}} x_{3}^{r\left(A_{n-1}-s_{n-1}\right)} z_{3}^{T}\right],
\end{aligned}
$$

where the summation runs over all integers $\tau_{0}, \ldots, \tau_{n-2}$ satisfying (16) and

$$
T=s^{\prime} s_{n-1}-t^{\prime} s_{n-2}=(r s-t) s_{n-1}-s s_{n-2} .
$$

Using Lemma 3.12(a), we see that the exponent of $x_{3}$ is equal to $A_{n}-r s_{n-1}$. Then substituting $\left(x_{2}^{r}+z_{3}^{s}\right) / x_{1}$ for $x_{3}$, we get

$x_{n+2}^{p} x_{n+1}^{q}$
$\quad=x_{2}^{-A_{n-1}} \sum_{\tau_{0}, \tau_{1}, \ldots, \tau_{n-2}}\left[\left(\prod_{i=0}^{n-2}\left[\begin{array}{c}A_{i+1}-r s_{i} \\ \tau_{i}\end{array}\right]\right) x_{2}^{r s_{n-2}}\left(\frac{x_{2}^{r}+z^{s}}{x}{ }_{1}\right)^{A_{n}-r s_{n-1}} z_{3}^{T}\right]$.

Expanding $\left(x_{2}^{r}+z^{s}\right)^{A_{n}-r s_{n-1}}$ yields

$$
\begin{aligned}
x_{1}^{-A_{n}} x_{2}^{-A_{n-1}} \sum_{\tau_{0}, \ldots, \tau_{n-2}}\left(\prod_{i=0}^{n-2}\left[\begin{array}{c}
A_{i+1}-r s_{i} \\
\tau_{i}
\end{array}\right]\right) \\
\times x_{2}^{r s_{n-2}} \sum_{\tau_{n-1} \in \mathbb{Z}}\left[\begin{array}{c}
A_{n}-r s_{n-1} \\
\tau_{n-1}
\end{array}\right] x_{1}^{r s_{n-1}}\left(x_{2}^{r}\right)^{A n-r s_{n-1}-\tau_{n-1}} z_{3}^{T+s \tau_{n-1}} .
\end{aligned}
$$


Note that $T+s \tau_{n-1}=s s_{n}-t s_{n-1}$, by Lemma 3.15. Combining the sums, we get

$$
\begin{aligned}
x_{1}^{-A_{n}} x_{2}^{-A_{n-1}} \sum_{\tau_{0}, \tau_{1}, \ldots, \tau_{n-2} ; \tau_{n-1} \in \mathbb{Z}} & \left(\prod_{i=0}^{n-1}\left[\begin{array}{c}
A_{i+1}-r s_{i} \\
\tau_{i}
\end{array}\right]\right) \\
\times & x_{1}^{r s_{n-1}} x_{2}^{r s_{n-2}}\left(x_{2}^{r}\right)^{A_{n}-r s_{n-1}-\tau_{n-1}} z_{3}^{s s_{n}-t s_{n-1}}
\end{aligned}
$$

and, by Lemma 3.15 , this is equal to

(20)

$$
x_{1}^{-A_{n}} x_{2}^{-A_{n-1}} \sum_{\tau_{0}, \tau_{1}, \ldots, \tau_{n-2} ; \tau_{n-1} \in \mathbb{Z}}\left(\prod_{i=0}^{n-1}\left[\begin{array}{c}
A_{i+1}-r s_{i} \\
\tau_{i}
\end{array}\right]\right) x_{1}^{r s_{n-1}} x_{2}^{r\left(A_{n}-s_{n}\right)} z_{3}^{s s_{n}-t s_{n-1}} .
$$

Remember that $\tau_{0}, \ldots, \tau_{n-2}$ satisfy (16).

Proposition 3.25 below implies that even if we impose the additional condition on $\tau_{n-2}$ and $\tau_{n-1}$ that

$$
\begin{aligned}
& \left(s_{n}-s_{n}^{\prime}\right) A_{n-1}-\left(s_{n-1}-s_{n-1}^{\prime}\right) A_{n} \geq 0 \\
& \qquad \text { for all }\left(\tau_{0}^{\prime}, \ldots, \tau_{n-1}^{\prime}\right) \in \mathcal{L}_{\max }\left(\tau_{0}, \ldots, \tau_{n-2}\right)
\end{aligned}
$$

the value of the expression for $x_{n+2}^{p} x_{n+1}^{q}$ in (20) does not change. From now on we impose the condition (21). On the other hand, in order to prove that Theorem 3.17 holds for $n+1$, we only need to show that $\tau_{n-2}$ can be limited to run over $0 \leq \tau_{n-2} \leq A_{n-1}-r s_{n-2}$. So we want to show that, for a fixed sequence $\left(\tau_{0}^{\prime}, \ldots, \tau_{n-2}^{\prime}, \tau_{n-1}^{\prime}\right)$,

$$
\sum_{\tau_{0}, \tau_{1}, \ldots, \tau_{n-1}}\left[\left(\prod_{i=0}^{n-1}\left[\begin{array}{c}
A_{i+1}-r s_{i} \\
\tau_{i}
\end{array}\right]\right) x_{1}^{r s_{n-1}} x_{2}^{r\left(A_{n}-s_{n}\right)}\right]=0
$$

where the summation runs over all integers $\tau_{0}, \ldots, \tau_{n-1}$ satisfying

$$
\left\{\begin{array}{l}
\text { (a) }\left(\tau_{0}^{\prime}, \ldots, \tau_{n-2}^{\prime}\right) \in \mathcal{L}_{\max }\left(\tau_{0}, \ldots, \tau_{n-2}\right) \\
\text { (b) } 0 \leq \tau_{i} \leq A_{i+1}-r s_{i}(0 \leq i \leq n-3) \\
\text { (c) }\left(s_{n-1}-s_{n-1}^{\prime}\right) A_{n-2}-\left(s_{n-2}-s_{n-2}^{\prime}\right) A_{n-1} \geq 0 \\
\text { (d) }\left(\tau_{0}^{\prime}, \ldots, \tau_{n-1}^{\prime}\right) \in \mathcal{L}_{\max }\left(\tau_{0}, \ldots, \tau_{n-1}\right) \\
\text { (e) } \tau_{n-2} \leq A_{n-1}-r s_{n-2}<0, \text { and } \\
\text { (f) }\left(s_{n}-s_{n}^{\prime}\right) A_{n-1}-\left(s_{n-1}-s_{n-1}^{\prime}\right) A_{n} \geq 0
\end{array}\right.
$$

To do so, it is sufficient to show that $\left[\begin{array}{c}c A_{n}-r s_{n-1} \\ \tau_{n-1}\end{array}\right]=0$ for every $\tau_{n-1}$, because then each summand in (22) is zero. This purely algebraic result is proved in Lemma 3.24 below. 
Assuming Lemma 3.24 and Proposition 3.25, we have proved that $x_{n+2}^{p} x_{n+1}^{q}$

$$
=x_{1}^{-A_{n}} x_{2}^{-A_{n-1}} \sum_{\tau_{0}, \tau_{1}, \ldots, \tau_{n-1}}\left[\left(\prod_{i=0}^{n-1}\left[\begin{array}{c}
A_{i+1}-r s_{i} \\
\tau_{i}
\end{array}\right]\right) x_{1}^{r s_{n-1}} x_{2}^{r\left(A_{n}-s_{n}\right)} z_{3}^{s s_{n}-t s_{n-1}}\right],
$$

where the summation runs over all integers $\tau_{0}, \ldots, \tau_{n-1}$ satisfying

$$
\left\{\begin{array}{l}
0 \leq \tau_{i} \leq A_{i+1}-r s_{i}(0 \leq i \leq n-2), \\
\left(s_{n-1}-s_{n-1}^{\prime}\right) A_{n-2}-\left(s_{n-2}-s_{n-2}^{\prime}\right) A_{n-1} \geq 0, \\
\left(s_{n}-s_{n}^{\prime}\right) A_{n-1}-\left(s_{n-1}-s_{n-1}^{\prime}\right) A_{n} \geq 0,
\end{array}\right.
$$

for all $\left(\tau_{0}^{\prime}, \ldots, \tau_{n-1}^{\prime}\right) \in \mathcal{L}_{\max }\left(\tau_{0}, \ldots, \tau_{n-2}\right)$. Therefore it only remains to show that we do not need to require the second condition in (24). Using Lemmas 3.15 and $3.12(\mathrm{a})$, we see that

$$
\begin{aligned}
s_{n-1} A_{n-2}-s_{n-2} A_{n-1} & \stackrel{3.15}{=}\left(r s_{n-2}-s_{n-3}+\tau_{n-2}\right) A_{n-2}-s_{n-2} A_{n-1} \\
& \stackrel{3.12}{=}\left(s_{n-2} A_{n-3}-s_{n-3} A_{n-2}\right)+\tau_{n-2} A_{n-2} .
\end{aligned}
$$

Iterating this argument, we get

$$
s_{n-1} A_{n-2}-s_{n-2} A_{n-1}=\left(s_{2} A_{1}-s_{1} A_{2}\right)+\sum_{i=2}^{n-2} \tau_{i} A_{i},
$$

which means

$$
\tau_{1} p-\tau_{0} q+\sum_{i=1}^{n-2} \tau_{i} A_{i} \geq \tau_{1} p-\tau_{0} q
$$

because $s_{1}=\tau_{0}, s_{2}=r \tau_{0}+\tau_{1}, A_{1}=p$ and $A_{2}=r p+q$. Thus

$$
s_{n-1} A_{n-2}-s_{n-2} A_{n-1} \geq \tau_{1} p-\tau_{0} q .
$$

Our next goal is to estimate $-s_{n-1}^{\prime} A_{n-2}+s_{n-2}^{\prime} A_{n-1}$. Let $k$ be as in Definition 3.16 , so that $0 \leq k \leq p$ and $s_{n-1}^{\prime}=k c_{n}$ and $s_{n-2}^{\prime}=k c_{n-1}$. Then

$$
\begin{aligned}
(26)-s_{n-1}^{\prime} A_{n-2}+s_{n-2}^{\prime} A_{n-1} & =k\left(-c_{n} A_{n-2}+c_{n-1} A_{n-1}\right) \\
& =k\left(-p c_{n} c_{n-1}-q c_{n} c_{n-2}+p c_{n} c_{n-1}+q c_{n-1}^{2}\right) \\
& =k q\left(-c_{n} c_{n-2}+c_{n-1}^{2}\right)=k q,
\end{aligned}
$$

where the second equality follows from the definition $A_{i}=p c_{i+1}+q c_{i}$, and the last equality holds by Lemma 3.1. On the other hand, $s_{n-2}^{\prime}$ is defined as $s_{n-2}^{\prime}=$ $c_{n-1} \tau_{0}^{\prime}+\sum_{j=1}^{n-3} c_{n-1-j} \tau_{j}^{\prime}$, which implies that $k c_{n-1}=s_{n-2}^{\prime} \geq c_{n-1} \tau_{0}^{\prime}$, and thus $k \geq \tau_{0}^{\prime}$. Moreover, $\tau_{0}=\tau_{0}^{\prime}$ by definition of $\mathcal{L}_{\max }\left(\tau_{0}, \tau_{1}, \ldots, \tau_{n-2}\right)$, and thus $(26)$ implies

$$
-s_{n-1}^{\prime} A_{n-2}+s_{n-2}^{\prime} A_{n-1} \geq \tau_{0} q
$$


Adding this to (25) we get

$$
\left(s_{n-1}-s_{n-1}^{\prime}\right) A_{n-2}-\left(s_{n-2}-s_{n-2}^{\prime}\right) A_{n-1} \geq 0,
$$

hence the second condition in (24) is always satisfied.

This completes the proof of Theorem 3.17 modulo Lemma 3.24 and Proposition 3.25 below.

Lemma 3.24. Assume conditions (23). Then

$$
\left[\begin{array}{c}
A_{n}-r s_{n-1} \\
\tau_{n-1}
\end{array}\right]=0 .
$$

Proof. By definition of the modified binomial coefficient, it suffices to show that $A_{n}-r s_{n-1}<\tau_{n-1}$.

On the one hand, we have

$$
A_{n-3}\left(s_{n-2}-s_{n-2}^{\prime}\right)-A_{n-2}\left(A_{n-1}-s_{n-1}+s_{n-1}^{\prime}\right)
$$

3.12(a)

$$
\begin{aligned}
& \stackrel{12}{=} r A_{n-2}\left(s_{n-2}-s_{n-2}^{\prime}\right)-A_{n-1}\left(s_{n-2}-s_{n-2}^{\prime}\right)-A_{n-2}\left(A_{n-1}-s_{n-1}+s_{n-1}^{\prime}\right) \\
& =A_{n-2} r s_{n-2}-r A_{n-2} s_{n-2}^{\prime}-A_{n-1}\left(s_{n-2}-s_{n-2}^{\prime}\right)-A_{n-2}\left(A_{n-1}-s_{n-1}+s_{n-1}^{\prime}\right) \\
& >A_{n-2} A_{n-1}-r A_{n-2} s_{n-2}^{\prime}-A_{n-1}\left(s_{n-2}-s_{n-2}^{\prime}\right)-A_{n-2}\left(A_{n-1}-s_{n-1}+s_{n-1}^{\prime}\right) \\
& \text { (since } A_{n-1}-r s_{n-2}<0 \text { ) } \\
& =-r A_{n-2} s_{n-2}^{\prime}+\left(s_{n-1}-s_{n-1}^{\prime}\right) A_{n-2}-\left(s_{n-2}-s_{n-2}^{\prime}\right) A_{n-1} \\
& \text { (23)(c) } \\
& \geq-r A_{n-2} s_{n-2}^{\prime} \text {. }
\end{aligned}
$$

Thus

$$
s_{n-2}-s_{n-2}^{\prime}>\left(A_{n-2}\left(A_{n-1}-s_{n-1}+s_{n-1}^{\prime}\right)-r A_{n-2} s_{n-2}^{\prime}\right) / A_{n-3} .
$$

Then

$$
\begin{aligned}
& \stackrel{(28)}{<} A_{n-2}\left(s_{n-1}-s_{n-1}^{\prime}\right)-A_{n-1} \frac{A_{n-2}\left(A_{n-1}-s_{n-1}+s_{n-1}^{\prime}\right)-r A_{n-2} s_{n-2}^{\prime}}{A_{n-3}} \\
& =A_{n-2}\left(s_{n-1}-s_{n-1}^{\prime}-\frac{A_{n-1}}{A_{n-3}}\left(A_{n-1}-s_{n-1}+s_{n-1}^{\prime}\right)\right)+\frac{r A_{n-1} A_{n-2} s_{n-2}^{\prime}}{A_{n-3}} \\
& =A_{n-2}\left(A_{n-1}-\left(1+\frac{A_{n-1}}{A_{n-3}}\right)\left(A_{n-1}-s_{n-1}+s_{n-1}^{\prime}\right)\right)+\frac{r A_{n-1} A_{n-2} s_{n-2}^{\prime}}{A_{n-3}} \\
& \stackrel{3.12(\mathrm{a})}{=} A_{n-2}\left(A_{n-1}-\left(1+\frac{A_{n-1}}{A_{n-3}}\right) \frac{A_{n-2}+A_{n}-r s_{n-1}+r s_{n-1}^{\prime}}{r}\right)+\frac{r A_{n-1} A_{n-2} s_{n-2}^{\prime}}{A_{n-3}} .
\end{aligned}
$$


Now, aiming for contradiction, suppose that $A_{n}-r s_{n-1} \geq 0$. Then

$$
\begin{aligned}
& \mathrm{RHS}(29) \\
& \quad \leq A_{n-2}\left(A_{n-1}-\frac{A_{n-3}+A_{n-1}}{A_{n-3}} \frac{A_{n-2}+r s_{n-1}^{\prime}}{r}\right)+\frac{r A_{n-1} A_{n-2} s_{n-2}^{\prime}}{A_{n-3}} \\
& \stackrel{3.12(\mathrm{a})}{=} A_{n-2}\left(A_{n-1}-\frac{r A_{n-2}}{A_{n-3}} \frac{A_{n-2}+r s_{n-1}^{\prime}}{r}\right)+\frac{r A_{n-1} A_{n-2} s_{n-2}^{\prime}}{A_{n-3}} \\
& \quad=A_{n-2}\left(A_{n-1}-\frac{A_{n-2}^{2}}{A_{n-3}}\right)-r \frac{A_{n-2}^{2}}{A_{n-3}} s_{n-1}^{\prime}+\frac{r A_{n-1} A_{n-2} s_{n-2}^{\prime}}{A_{n-3}} \\
& \quad=\frac{A_{n-2}}{A_{n-3}}\left(A_{n-1} A_{n-3}-A_{n-2}^{2}\right)-r \frac{A_{n-2}}{A_{n-3}}\left(A_{n-2} s_{n-1}^{\prime}-A_{n-1} s_{n-2}^{\prime}\right) .
\end{aligned}
$$

By Lemma 3.12(b) and the definition of $A_{i}$, this is equal to

$$
\frac{A_{n-2}}{A_{n-3}}\left(-p^{2}-q^{2}-r p q\right)-r \frac{A_{n-2}}{A_{n-3}}\left(\left(p c_{n-1}+q c_{n-2}\right) s_{n-1}^{\prime}-\left(p c_{n}+q c_{n-1}\right) s_{n-2}^{\prime}\right) .
$$

Let $k$ be as in Definition 3.16 ; then $k c_{n}=s_{n-1}^{\prime}$ and $k c_{n-1}=s_{n-2}^{\prime}$, and using Lemma 3.1, we get

$$
\frac{A_{n-2}}{A_{n-3}}\left(-p^{2}-q^{2}-r p q\right)+r k q \frac{A_{n-2}}{A_{n-3}},
$$

and, since $k \leq p$, this is less than or equal to

$$
\frac{A_{n-2}}{A_{n-3}}\left(-p^{2}-q^{2}-r p q\right)+r p q \frac{A_{n-2}}{A_{n-3}} \leq 0,
$$

which contradicts $\left(s_{n-1}-s_{n-1}^{\prime}\right) A_{n-2}-\left(s_{n-2}-s_{n-2}^{\prime}\right) A_{n-1} \geq 0$. Hence

$$
A_{n}-r s_{n-1}<0 \text {. }
$$

Next we show that $s_{n-2}>A_{n}-s_{n}$. Suppose to the contrary that $s_{n-2} \leq$ $A_{n}-s_{n}$. Then

$$
\begin{aligned}
A_{n-1}-r s_{n-2} & \geq A_{n-1}-r\left(A_{n}-s_{n}\right) \stackrel{(23)(\mathrm{f})}{\geq} A_{n-1}-r \frac{\left(A_{n-1}-s_{n-1}+s_{n-1}^{\prime}\right) A_{n}}{A_{n-1}}+r s_{n}^{\prime} \\
& \stackrel{3.12(\mathrm{~b})}{=} \frac{\left(p^{2}+q^{2}+r p q\right)}{A_{n-1}}+\frac{A_{n} A_{n-2}}{A_{n-1}}-r \frac{\left(A_{n-1}-s_{n-1}+s_{n-1}^{\prime}\right) A_{n}}{A_{n-1}}+r s_{n}^{\prime} \\
& \stackrel{3.12(\mathrm{a})}{=} \frac{\left(p^{2}+q^{2}+r p q\right)}{A_{n-1}}+\frac{A_{n}}{A_{n-1}}\left(r s_{n-1}-A_{n}-r s_{n-1}^{\prime}+\frac{A_{n-1}}{A_{n}} r s_{n}^{\prime}\right) \\
= & \frac{p^{2}+q^{2}}{A_{n-1}}+\frac{A_{n}}{A_{n-1}}\left(r s_{n-1}-A_{n}\right)+\frac{r}{A_{n-1}}\left(p q-A_{n} s_{n-1}^{\prime}+A_{n-1} s_{n}^{\prime}\right) \\
& \stackrel{3.16}{=} \frac{p^{2}+q^{2}}{A_{n-1}}+\frac{A_{n}}{A_{n-1}}\left(r s_{n-1}-A_{n}\right)+\frac{r}{A_{n-1}}\left(p q-A_{n} k c_{n}+A_{n-1} k c_{n+1}\right) .
\end{aligned}
$$


Now $k c_{n} A_{n}-k c_{n+1} A_{n-1}=k c_{n}\left(p c_{n+1}+q c_{n}\right)-k c_{n+1}\left(p c_{n}+q c_{n-1}\right)=k q\left(c_{n}^{2}-\right.$ $\left.c_{n+1} c_{n-1}\right)=k q$, where the last equation holds because of Lemma 3.1. Thus

$$
\begin{aligned}
A_{n-1}-r s_{n-2} & \geq \frac{p^{2}+q^{2}}{A_{n-1}}+\frac{A_{n}}{A_{n-1}}\left(r s_{n-1}-A_{n}\right)+\frac{r}{A_{n-1}}(q(p-k)) \\
3.16 \text { and }(23)(\mathrm{d}) & \frac{p^{2}+q^{2}}{A_{n-1}}+\frac{A_{n}}{A_{n-1}}\left(r s_{n-1}-A_{n}\right) \stackrel{(31)}{>} 0,
\end{aligned}
$$

which contradicts $A_{n-1}-r s_{n-2}<0$ in (23). Thus $s_{n-2}>A_{n}-s_{n}$, so we have

$$
A_{n}-r s_{n-1}<s_{n}+s_{n-2}-r s_{n-1}=\tau_{n-1},
$$

which gives $\left[\begin{array}{c}c A_{n}-r s_{n-1} \\ \tau_{n-1}\end{array}\right]=0$.

Proposition 3.25. Let $a$ and $b$ be nonnegative integers satisfying

$$
\sum_{\substack{\tau_{0}, \tau_{1}, \ldots, \tau_{n-2} \\
s_{n-1}=a, s_{n-2}=b}} \prod_{i=0}^{n-2}\left[\begin{array}{c}
A_{i+1}-r s_{i} \\
\tau_{i}
\end{array}\right] \neq 0 .
$$

Let $\tau_{0}, \ldots, \tau_{n-2}$ satisfy $s_{n-1}=a, s_{n-2}=b$. Then for any $\left(\tau_{0}^{\prime}, \ldots, \tau_{n-2}^{\prime}\right) \in$ $\mathcal{L}_{\max }\left(\tau_{0}, \ldots, \tau_{n-2}\right)$, we have

$$
\left(s_{n-1}-s_{n-1}^{\prime}\right) A_{n-2}-\left(s_{n-2}-s_{n-2}^{\prime}\right) A_{n-1} \geq 0 .
$$

Proof. We use Theorem 3.13. Let $\mathcal{D}_{i, 1}$ (respectively $\mathcal{D}_{i, 2}$ ) be the set of horizontal (resp. vertical) edges in the $i$-th Dyck path in $\left(\mathcal{D}^{c_{n} \times c_{n-1}}\right)^{p} \times\left(\mathcal{D}^{c_{n-1} \times c_{n-2}}\right)^{q}$.

Choose a collection of compatible pairs

$$
\begin{aligned}
\beta & =\left(\beta_{1}, \ldots, \beta_{p+q}\right) \\
& =\left(\left(S_{1,1}, S_{1,2}\right), \ldots,\left(S_{p+q, 1}, S_{p+q, 2}\right)\right) \text { in }\left(\mathcal{D}^{c_{n} \times c_{n-1}}\right)^{p} \times\left(\mathcal{D}^{c_{n-1} \times c_{n-2}}\right)^{q}
\end{aligned}
$$

such that $\sum_{i=1}^{p+q}\left|S_{i, 2}\right|=s_{n-2}$ and $p c_{n}+q c_{n-1}-\sum_{i=1}^{p+q}\left|S_{i, 1}\right|=s_{n-1}$, and such that $\beta$ has the maximal number, say $w$, of copies of $\left(\emptyset, \mathcal{D}_{2}\right)$ in $\mathcal{D}^{c_{n} \times c_{n-1}}$. Say $\beta_{i_{1}}=\cdots=\beta_{i_{w}}=\left(\emptyset, \mathcal{D}_{2}\right)$ for some $1 \leq i_{1}<\cdots<i_{w} \leq p$. Since $\left|\mathcal{D}_{2}\right|=c_{n-1}$ we see that

$$
w=\min \left(\left\lfloor\frac{s_{n-2}}{c_{n-1}}\right\rfloor, p\right) .
$$

On the other hand, by Definition 3.16 and Remark 3.19, $s_{n-2}^{\prime}=k c_{n-1}$ and $s_{n-1}^{\prime}=$ $k c_{n}$ with $k=w$ because $\left(\tau_{0}^{\prime}, \tau_{1}^{\prime}, \ldots, \tau_{n-2}^{\prime}\right) \in \mathcal{L}_{\max }$. Therefore

$$
\begin{aligned}
\sum_{i \in\{1, \ldots, p+q\} \backslash\left\{i_{1}, \ldots, i_{w}\right\}}\left|\beta_{i}\right|_{2} & =s_{n-1}-w c_{n}=s_{n-1}-s_{n-1}^{\prime}, \\
\sum_{i \in\{1, \ldots, p+q\} \backslash\left\{i_{1}, \ldots, i_{w}\right\}}\left|\beta_{i}\right|_{1} & =s_{n-2}-w c_{n-1}=s_{n-2}-s_{n-2}^{\prime},
\end{aligned}
$$


where $\left|\beta_{i}\right|_{2}$ denotes $\left|\mathcal{D}_{i, 1}\right|-\left|S_{i, 1}\right|$ and $\left|\beta_{i}\right|_{1}$ denotes $\left|S_{i, 2}\right|$. Hence (32) is equivalent to

$$
\sum_{i \in\{1, \ldots, p+q\} \backslash\left\{i_{1}, \ldots, i_{w}\right\}}\left(A_{n-2}\left|\beta_{i}\right|_{2}-A_{n-1}\left|\beta_{i}\right|_{1}\right) \geq 0 .
$$

First we show that

$$
\sum_{i=p+1}^{p+q}\left(A_{n-2}\left|\beta_{i}\right|_{2}-A_{n-1}\left|\beta_{i}\right|_{1}\right) \geq 0 .
$$

Due to Lemma 3.8, if $p<i \leq p+q$ then either $\beta_{i}=\left(\mathcal{D}_{i, 1}, \emptyset\right)$ or $\beta_{i}=\left(\emptyset, \mathcal{D}_{i, 2}\right)$ gives the minimum of $A_{n-2}\left|\beta_{i}\right|_{2}-A_{n-1}\left|\beta_{i}\right|_{1}$. If $\beta_{i}=\left(\mathcal{D}_{i, 1}, \emptyset\right)$ then clearly $A_{n-2}\left|\beta_{i}\right|_{2}-$ $A_{n-1}\left|\beta_{i}\right|_{1}=0$. If $\beta_{i}=\left(\emptyset, \mathcal{D}_{i, 2}\right)$ then

$$
\begin{aligned}
A_{n-2}\left|\beta_{i}\right|_{2}-A_{n-1}\left|\beta_{i}\right|_{1} & =A_{n-2} c_{n-1}-A_{n-1} c_{n-2} \\
& =\left(p c_{n-1}+q c_{n-2}\right) c_{n-1}-\left(p c_{n}+q c_{n-1}\right) c_{n-2} \\
& =p\left(c_{n-1}^{2}-c_{n} c_{n-2}\right)=p \geq 0 .
\end{aligned}
$$

Next we show that

$$
\sum_{i \in\{1, \ldots, p\} \backslash\left\{i_{1}, \ldots, i_{w}\right\}}\left(A_{n-2}\left|\beta_{i}\right|_{2}-A_{n-1}\left|\beta_{i}\right|_{1}\right) \geq 0 .
$$

If $p=0$ then there is nothing to show. Suppose that $p \geq 1$. Again using Lemma 3.8, we see that if $1 \leq i \leq p$ and $\beta_{i} \neq\left(\emptyset, \mathcal{D}_{i, 2}\right)$ then

$$
c_{n-2}\left|\beta_{i}\right|_{2}-c_{n-1}\left|\beta_{i}\right|_{1}>c_{n-2} c_{n}-c_{n-1}^{2}=-1,
$$

so $c_{n-2}\left|\beta_{i}\right|_{2}-c_{n-1}\left|\beta_{i}\right|_{1} \geq 0$. Also $c_{n-1}\left|\beta_{i}\right|_{2}-c_{n}\left|\beta_{i}\right|_{1} \geq c_{n-1} c_{n}-c_{n} c_{n-1}=0$. Thus

$$
\begin{aligned}
A_{n-2}\left|\beta_{i}\right|_{2}-A_{n-1}\left|\beta_{i}\right|_{1} & =\left(p c_{n-1}+q c_{n-2}\right)\left|\beta_{i}\right|_{2}-\left(p c_{n}+q c_{n-1}\right)\left|\beta_{i}\right|_{1} \\
& =p\left(c_{n-1}\left|\beta_{i}\right|_{2}-c_{n}\left|\beta_{i}\right|_{1}\right)+q\left(c_{n-2}\left|\beta_{i}\right|_{2}-c_{n-1}\left|\beta_{i}\right|_{1}\right) \geq 0 .
\end{aligned}
$$

\section{§3.6. Divisibility in rank 2}

We end this section with the following rank 2 result which we will need in Section 4 for the rank 3 case.

Theorem 3.26. Let $a \geq A_{n} / r$ be an integer. Then

$$
\sum_{\substack{\tau_{0}, \tau_{1}, \ldots, \tau_{n-2} \\
s_{n-1}=a}} \prod_{i=0}^{n-2}\left[\begin{array}{c}
A_{i+1}-r s_{i} \\
\tau_{i}
\end{array}\right] x_{1}^{r s_{n-2}-A_{n-1}} x_{2}^{r\left(A_{n-1}-a\right)-A_{n-2}}
$$

is divisible by $\left(1+x_{1}^{r}\right)^{r a-A_{n}}$ and the resulting quotient has nonnegative coefficients. 
Proof. Using Theorem 3.17 with $z_{3}=1$, we see that $x_{n+1}^{p} x_{n}^{q}$ is equal to

$$
\sum_{\tau_{0}, \tau_{1}, \ldots, \tau_{n-2}}\left(\prod_{i=0}^{n-2}\left[\begin{array}{c}
A_{i+1}-r s_{i} \\
\tau_{i}
\end{array}\right]\right) x_{1}^{r s_{n-2}-A_{n-1}} x_{2}^{A_{n}-r s_{n-1}} .
$$

On the other hand, using Theorem 3.17 to express $x_{n+1}^{p} x_{n}^{q}$ in the cluster $\left(x_{0}=\right.$ $\left.\left(x_{1}^{r}+1\right) / x_{2}, x_{1}\right)$, we get

$$
\sum_{\tau_{0}, \tau_{1}, \ldots, \tau_{n-2}, \tau_{n-1}}\left(\prod_{i=0}^{n-1}\left[\begin{array}{c}
A_{i+1}-r s_{i} \\
\tau_{i}
\end{array}\right]\right)\left(\frac{x_{1}^{r}+1}{x}{ }_{2}\right)^{r s_{n-1}-A_{n}} x_{1}^{A_{n+1}-r s_{n}} .
$$

Since the positivity conjecture is known to hold for rank 2 , it follows that all the sums of products of modified binomial coefficients in this expression are positive. Now the result follows by fixing $s_{n-1}=a$.

\section{$\S 4$. Rank 3}

\section{§4.1. Nonacyclic mutation classes of rank 3}

In this subsection we collect some basic results on quivers of rank 3 which are not mutation equivalent to an acyclic quiver. First let us recall how mutations act on a rank 3 quiver. Given a quiver

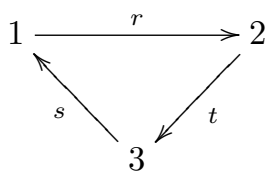

where $r, s, t \geq 1$ denote the numbers of arrows, its mutation in 1 is the quiver

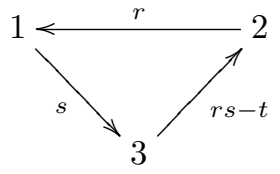

where we agree that if $r s-t<0$ then there are $|r s-t|$ arrows from 2 to 3.

Lemma 4.1. Let $\mathcal{A}$ be a nonacyclic cluster algebra of rank 3 with initial quiver $Q$ equal to

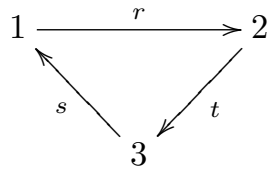

where $r, s, t \geq 1$ denote the numbers of arrows. Then 
(i) $r, s, t \geq 2$;

(ii) applying to $Q$ a mutation sequence at the vertices $1,2,1,2,1,2, \ldots$ consisting of $n$ mutations yields the quiver

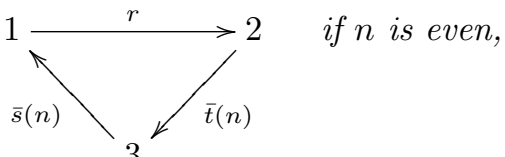

$\bar{s}(n)=c_{n+2}^{[r]} s-c_{n+1}^{[r]} t \quad$ and $\quad \bar{t}(n)=c_{n+1}^{[r]} s-c_{n}^{[r]} t$.

(iii) $c_{n+1}^{[r]} s-c_{n}^{[r]} t \geq 2$ for all $n \geq 1$.

Proof. (i) This has been shown in $[3]^{1}$, but we include a proof for convenience. Suppose that one of $r, s, t$ is less than 2. Without loss of generality, we may suppose $r<2$ and $s \leq t$. Since $Q$ is not acyclic, $r$ cannot be zero, whence $r=1$. But then mutating $Q$ at vertex 1 would produce the following acyclic quiver:

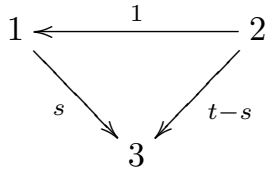

(ii) We proceed by induction on $n$. For $n=1$, the quiver obtained from $Q$ by mutation at 1 is the following:

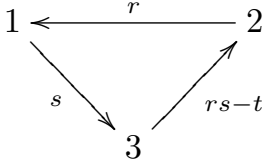

and for $n=2$, the quiver obtained from $Q$ by mutation at 1 and 2 is the following:

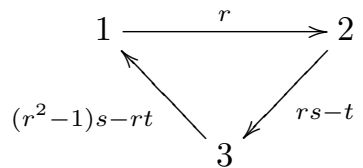

In both cases, the result follows from $c_{1}^{[r]}=0, c_{2}^{[r]}=1, c_{3}^{[r]}=r, c_{4}^{[r]}=r^{2}-1$.

\footnotetext{
${ }^{1}$ It is shown there that $A$ is nonacyclic if and only if $r, s, t \geq 2$ and $r^{2}+s^{2}+t^{2}-r s t \leq 4$.
} 
Suppose that $n>2$. If $n$ is odd then by induction we know that the quiver we are considering is obtained by mutating the following quiver at vertex 1 :

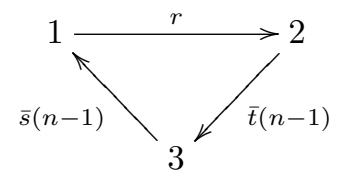

and the result follows from $\bar{t}(n)=\bar{s}(n-1)$ and

$$
r \bar{s}(n-1)-\bar{t}(n-1)=r c_{n+1}^{[r]} s-r c_{n}^{[r]} t-c_{n}^{[r]} s+c_{n-1}^{[r]} t=c_{n+2}^{[r]} s-c_{n+1}^{[r]} t=\bar{s}(n) .
$$

If $n$ is even then the proof is similar. (iii) follows from (i) and (ii).

\section{$\S 4.2$. Positivity}

In this section, we prove the positivity conjecture in rank 3.

Theorem 4.2. Let $\mathcal{A}(Q)$ be a skew-symmetric coefficient-free cluster algebra of rank 3 with initial cluster $\mathbf{x}$ and let $\mathbf{x}_{t_{0}}$ be any cluster. Then the Laurent expansion of any cluster variable in $\mathbf{x}_{t_{0}}$ with respect to the cluster $\mathbf{x}$ has nonnegative coefficients.

The remainder of this section is devoted to the proof of this theorem.

If $\mathcal{A}$ is acyclic then the theorem has been proved by Kimura and Qin [17]. We therefore assume that $\mathcal{A}$ is nonacyclic, but we point out that this is not a necessary assumption for our argument. Our methods would work also in the acyclic case, but restricting to the nonacyclic case considerably simplifies the exposition.

Since $\mathcal{A}$ is nonacyclic, every quiver $Q^{\prime}$ which is mutation equivalent to the initial quiver $Q$ has at least one oriented cycle. Since $Q^{\prime}$ has three vertices and no 2-cycles, this implies that every arrow in $Q^{\prime}$ lies in at least one oriented cycle.

Let $\mathbf{x}_{t_{0}}$ be an arbitrary cluster. Choose a finite sequence $\mu$ of mutations which transforms $\mathbf{x}_{t_{0}}$ into the initial cluster $\mathbf{x}$ and which is of minimal length among all such sequences. Each mutation in the sequence $\mu$ is a mutation at one of the three vertices 1,2 or 3 of the quiver, thus $\mu$ induces a finite sequence of vertices $\left(V_{1}, V_{2}, \ldots\right)$, where each $V_{i}$ is 1,2 or 3 . Since $\mu$ is of minimal length, it follows that $V_{i} \neq V_{i+1}$.

Let $e_{0,1}=V_{1}$ and $e_{0,2}=V_{2}$. Let $k_{1}>1$ be the least integer such that $V_{k_{1}} \neq e_{0,1}, e_{0,2}$, and denote by $t_{1}$ the seed obtained from $t_{0}$ after mutating at $V_{1}, \ldots, V_{k_{1}-1}$. Then let $e_{1,1}=V_{k_{1}-1}$ and $e_{1,2}=V_{k_{1}}$. Let $k_{2}>k_{1}$ be the least integer such that $V_{k_{2}} \neq e_{1,1}, e_{1,2}$, and denote by $t_{2}$ the seed obtained from $t_{0}$ after mutating 
at $V_{1}, \ldots, V_{k_{2}-1}$. Recursively, we define a sequence of seeds $\Sigma_{t_{0}}, \Sigma_{t_{1}}, \Sigma_{t_{2}}, \ldots, \Sigma_{t_{m}}$ along our sequence $\mu$ such that $\mathbf{x}_{t_{m}}=\mathbf{x}$ is the initial cluster and, for each $i$, the subsequence of $\mu$ between $t_{i}$ and $t_{i+1}$ is a sequence of mutations at two vertices.

The sequence of mutations can be visualized in the following diagram:

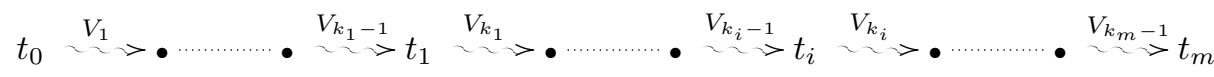

The main idea of our proof is to use our rank 2 formula from the previous section to compute the Laurent expansion of a cluster variable of $t_{0}$ in the cluster at $t_{1}$, then replace the cluster variables of $t_{1}$ in this expression by their Laurent expansions in the cluster $t_{2}$, which we can compute again because of our rank 2 formulas. Continuing this procedure we obtain, at least theoretically, a Laurent expansion in the initial cluster.

Fix an arbitrary $j \geq 0$, let $d=e_{j, 1}, e=e_{j, 2}$, and let $f$ be the integer such that $\{d, e, f\}=\{1,2,3\}$. Thus the sequence $\mu$ of mutations around the node $t_{j}$ is of the form

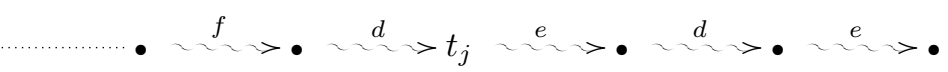

Let

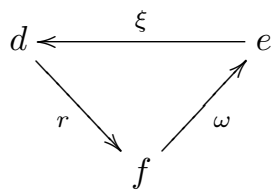

be the quiver at $t_{j}$, and use the notation $\left\{x_{1 ; t_{j}}, x_{2 ; t_{j}}, x_{3 ; t_{j}}\right\}$ for the cluster $\mathbf{x}_{t_{j}}$ at $t_{j}$. Let

$$
\widetilde{x_{d ; t_{j}}}=\frac{x_{e ; t_{j}}^{\xi}+x_{f ; t_{j}}^{r}}{x_{d ; t_{j}}}
$$

and

$$
\widetilde{\widetilde{x_{e ; t_{j}}}}=\frac{\widetilde{x_{d ; t_{j}}} \xi+x_{f ; t_{j}}^{r \xi-\omega}}{x_{e ; t_{j}}}
$$

Thus $\widetilde{x_{d ; t_{j}}}=\mu_{d}\left(x_{d ; t_{j}}\right)$ is the new cluster variable obtained by mutation of the cluster at $t_{j}$ in direction $d$ and $\widetilde{\widetilde{x_{e ; t_{j}}}}=\mu_{e} \mu_{d}\left(x_{e ; t_{j}}\right)$ is the new cluster variable obtained by mutation of the cluster at $t_{j}$ in direction $d$ and then $e$.

Theorem 4.2 follows easily from the following result. 
Theorem 4.3. For any $t_{i}, t_{j}$ with $i<j$, the Laurent expansion of the cluster monomial $x_{e_{i, 1} ; t_{i}}^{p} x_{e_{i, 2} ; t_{i}}^{q}$ in the cluster $\mathbf{x}_{t_{j}}$ is of the form

$$
\begin{aligned}
\sum_{u_{1} \in \mathbb{Z}, p_{1}, q_{1} \geq 0} C_{1 ; i \rightarrow j} x_{f ; t_{j}}^{u_{1}} \widetilde{x_{d ; t_{j}}} p_{1} \widetilde{x_{e ; t_{j}}} q_{1}+\sum_{u_{2} \in \mathbb{Z}, p_{2}, q_{2} \geq 0} C_{2 ; i \rightarrow j} x_{f ; t_{j}}^{u_{2}} \widetilde{x_{d ; t_{j}}} p^{2} x_{e ; t_{j}}^{q_{2}} \\
+\sum_{u_{3} \in \mathbb{Z}, p_{3}, q_{3} \geq 0} C_{3 ; i \rightarrow j} x_{f ; t_{j}}^{u_{3}} x_{d ; t_{j}}^{p_{3}} x_{e ; t_{j}}^{q_{3}},
\end{aligned}
$$

where

$$
\begin{aligned}
& C_{1 ; i \rightarrow j}=C_{1}\left(Q_{t_{j}}, V_{k_{i}}, \ldots, V_{k_{j}-1}, p, q ; u_{1}, p_{1}, q_{1}\right), \\
& C_{2 ; i \rightarrow j}=C_{2}\left(Q_{t_{j}}, V_{k_{i}}, \ldots, V_{k_{j}-1}, p, q ; u_{2}, p_{2}, q_{2}\right), \\
& C_{3 ; i \rightarrow j}=C_{3}\left(Q_{t_{j}}, V_{k_{i}}, \ldots, V_{k_{j}-1}, p, q ; u_{3}, p_{3}, q_{3}\right)
\end{aligned}
$$

are nonnegative integers which depend on $Q_{t_{j}}, V_{k_{i}}, \ldots, V_{k_{j}-1}, p, q, u_{1}, u_{2}, u_{3}, p_{1}, p_{2}$, $p_{3}, q_{1}, q_{2}, q_{3}$.

\section{$\S 4.3$. Proof of positivity}

Before proving Theorem 4.3, let us show that it implies Theorem 4.2.

Let $x$ be a cluster variable in $\mathbf{x}_{t_{0}}$. Using Theorem 4.3 with $i=0$ and $j=m-1$, we get an expression $\operatorname{LP}\left(x, t_{j}\right)$ for $x$ as a Laurent polynomial with nonnegative coefficients in which each summand is a quotient of a certain monomial in the variables $x_{f ; t_{j}}, \widetilde{x_{d ; t_{j}}}, \widetilde{\widetilde{x_{e ; t_{j}}}}, x_{e ; t_{j}}$ by a power of $x_{f ; t_{j}}$. In other words, only the variable $x_{f ; t_{j}}$ appears in the denominator of $L P\left(x, t_{j}\right)$.

Since the seed $t_{j}$ is obtained from the seed $t_{m}$ by a sequence of mutations at the vertices $d$ and $e$, we see that $x_{f ; t_{j}}=x_{f ; t_{m}}$ is one of the initial cluster variables and that the variables $x_{d ; t_{j}}, x_{e ; t_{j}} \widetilde{x_{d ; t_{j}}}, \widetilde{x_{e ; t_{j}}}$ are obtained from the initial cluster $\mathbf{x}_{t_{m}}$ by a sequence of mutations at $d$ and $e$. Therefore, in order to write $x$ as a Laurent polynomial in the initial cluster $\mathbf{x}_{t_{m}}$, we only need to compute the expansions for the variables $x_{d ; t_{j}}, x_{e ; t_{j}}, \widetilde{x_{d ; t_{j}}}, \widetilde{\widetilde{x_{e ; t_{j}}}}$ in the cluster $\mathbf{x}_{t_{m}}$ and substitute these expansions into $L P\left(x, t_{j}\right)$. But since the exponents $p_{i}, q_{i}$ of these variables are nonnegative, we know from Theorem 3.10 that these expansions are Laurent polynomials with nonnegative coefficients, and hence, after substitution into $\operatorname{LP}\left(x, t_{j}\right)$, we get an expansion for $x$ as a Laurent polynomial with nonnegative coefficients in the initial cluster $\mathbf{x}_{t_{m}}$.

\section{§4.4. Proof of Theorem 4.3}

We use induction on $j$. Suppose that the statement holds true for $j$, and we prove it for $j+1$. Note that $f=e_{j+1,2}$, since the rank is 3 . Without loss of generality, 
let $d=1$, so the sequence of mutations $\mu$ around the node $t_{j+1}$ is of the form

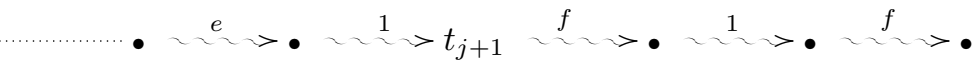

We analyze the three sums in the statement of Theorem 4.3 separately. For the first sum, thanks to Theorem 3.21 with $x_{1}=x_{1 ; t_{j+1}}, x_{2}=x_{e ; t_{j+1}}, x_{3}=\widetilde{x_{1 ; t_{j+1}}}$, $z_{3}=x_{f ; t_{j+1}}$, there exist nonnegative coefficients $\overline{\overline{C_{2 ; j \rightarrow j+1}}}$ and $\overline{\overline{C_{3 ; j \rightarrow j+1}}}$ such that

$$
\begin{aligned}
\sum_{u_{1} \in \mathbb{Z}, p_{1}, q_{1} \geq 0} C_{1 ; i \rightarrow j} x_{f ; t_{j}}^{u_{1}} \widetilde{x_{1 ; t_{j}}} p_{1} \widetilde{x_{e ; t_{j}}} q_{1}=\sum_{u_{1} \in \mathbb{Z}, p_{1}, q_{1} \geq 0} C_{1 ; i \rightarrow j} x_{f ; t_{j}}^{u_{1}} \\
\times\left(\begin{array}{c}
\sum_{v_{e}^{(1)} \in \mathbb{Z}, p_{2}^{(1)}, q_{2}^{(1)} \geq 0} \overline{\overline{C_{2 ; j \rightarrow j+1}}} x_{e ; t_{j+1}}^{v_{e}^{(1)}} \widetilde{x_{1 ; t_{j+1}}} p_{2}^{(1)} x_{f ; t_{j+1}}^{q_{2}^{(1)}} \\
+\sum_{w_{e}^{(1)} \in \mathbb{Z}, p_{3}^{(1)}, q_{3}^{(1)} \geq 0} \overline{\overline{C_{3 ; j \rightarrow j+1}}} x_{e ; t_{j+1}}^{w_{e}^{(1)}} x_{1 ; t_{j+1}}^{p_{3}^{(1)}} x_{f ; t_{j+1}}^{q_{3}^{(1)}}
\end{array}\right),
\end{aligned}
$$

where $\overline{\overline{C_{2 ; j \rightarrow j+1}}}$ depends on $v_{e}^{(1)}, p_{2}^{(1)}, q_{2}^{(1)}$, while $\overline{\overline{C_{3 ; j \rightarrow j+1}}}$ depends on $w_{e}^{(1)}$, $p_{3}^{(1)}, q_{3}^{(1)}$.

We can rewrite (34) as

$$
\begin{aligned}
\sum C_{1 ; i \rightarrow j} \overline{\overline{C_{2 ; j \rightarrow j+1}}} x_{e ; t_{j+1}}^{v_{e}^{(1)}} \widetilde{x_{1 ; t_{j+1}}} p_{2}^{(1)} x_{f ; t_{j+1}}^{u_{1}+q_{2}^{(1)}} \\
+\sum C_{1 ; i \rightarrow j} \overline{\overline{C_{3 ; j \rightarrow j+1}}} x_{e ; t_{j+1}}^{w_{e}^{(1)}} x_{1 ; t_{j+1}}^{p_{3}^{(1)}} x_{f ; t_{j+1}}^{u_{1}+q_{3}^{(1)}} .
\end{aligned}
$$

For the second sum, we have

$$
\begin{aligned}
& \sum_{u_{2} \in \mathbb{Z}, p_{2}, q_{2} \geq 0} C_{2 ; i \rightarrow j} x_{f ; t_{j}}^{u_{2}} \widetilde{x_{1 ; t_{j}}} p_{2} x_{e ; t_{j}}^{q_{2}}=\sum_{u_{2} \in \mathbb{Z}, p_{2}, q_{2} \geq 0} C_{2 ; i \rightarrow j} x_{f ; t_{j}}^{u_{2}} \\
& \times\left(\begin{array}{c}
\sum_{V_{e} \in \mathbb{Z}, p_{2}^{(2)}, q_{2}^{(2)} \geq 0} \overline{C_{2 ; j \rightarrow j+1}} x_{e ; t_{j+1}}^{v_{e}^{(2)}} \widetilde{x_{1 ; t_{j+1}}} p_{2}^{(2)} x_{f ; t_{j+1}}^{q_{2}^{(2)}} \\
+\sum_{w_{e}^{(2)} \in \mathbb{Z}, p_{3}^{(2)}, q_{3}^{(2)} \geq 0} \overline{C_{3 ; j \rightarrow j+1}} x_{e ; t_{j+1}}^{w_{e}^{(2)}} x_{1 ; t_{j+1}}^{p_{3}^{(2)}} x_{f ; t_{j+1}}^{q_{3}^{(2)}}
\end{array}\right),
\end{aligned}
$$

where $\overline{C_{2 ; j \rightarrow j+1}}$ depends on $v_{e}^{(2)}, p_{2}^{(2)}, q_{2}^{(2)}$, while $\overline{C_{3 ; j \rightarrow j+1}}$ depends on $w_{e}^{(2)}$, $p_{3}^{(2)}, q_{3}^{(2)}$.

We can rewrite $(36)$ as

$$
\begin{aligned}
\sum C_{2 ; i \rightarrow j} \overline{C_{2 ; j \rightarrow j+1}} x_{e ; t_{j+1}}^{v_{e}^{(2)}}{\widetilde{x_{1 ; t_{j+1}}}}_{p_{2}^{(2)}}^{x_{f ; t_{j+1}}^{u_{2}+q_{2}^{(2)}}} \\
+\sum C_{2 ; i \rightarrow j} \overline{C_{3 ; j \rightarrow j+1}} x_{e ; t_{j+1}}^{w_{e}^{(2)}} x_{1 ; t_{j+1}}^{p_{3}^{(2)}} x_{f ; t_{j+1}}^{u_{2}+q_{3}^{(2)}} .
\end{aligned}
$$


Similarly, for the third sum

$$
\begin{aligned}
\sum_{u_{3} \in \mathbb{Z}, p_{3}, q_{3} \geq 0} C_{3 ; i \rightarrow j} x_{f ; t_{j}}^{u_{3}} x_{1 ; t_{j}}^{p_{3}} x_{e ; t_{j}}^{q_{3}} & \sum_{u_{3} \in \mathbb{Z}, p_{3}, q_{3} \geq 0} C_{3 ; i \rightarrow j} x_{f ; t_{j}}^{u_{3}} \\
& \times\left(\begin{array}{c}
\sum_{v_{e}^{(3)} \in \mathbb{Z}, p_{2}^{(3)}, q_{2}^{(3)} \geq 0} C_{2 ; j \rightarrow j+1} x_{e ; t_{j+1}}^{v_{e}^{(3)}}{\widehat{x_{1 ; t_{j+1}}}}_{p_{2}^{(3)}} x_{f ; t_{j+1}}^{q_{2}^{(3)}} \\
+\sum_{w_{e}^{(3)} \in \mathbb{Z}, p_{3}^{(3)}, q_{3}^{(3)} \geq 0} C_{3 ; j \rightarrow j+1} x_{e ; t_{j+1}}^{w_{e}^{(3)}} x_{1 ; t_{j+1}}^{p_{3}^{(3)}} x_{f ; t_{j+1}}^{q_{3}^{(3)}}
\end{array}\right),
\end{aligned}
$$

where $C_{2 ; j \rightarrow j+1}$ depends on $v_{e}^{(3)}, p_{2}^{(3)}, q_{2}^{(3)}$, while $C_{3 ; j \rightarrow j+1}$ depends on $w_{e}^{(3)}$, $p_{3}^{(3)}, q_{3}^{(3)}$.

We can rewrite $(38)$ as

$$
\begin{aligned}
& \sum C_{3 ; i \rightarrow j} C_{2 ; j \rightarrow j+1} x_{e ; t_{j+1}}^{v_{e}^{(3)}} \widetilde{x_{1 ; t_{j+1}}} p_{2}^{(3)} x_{f ; t_{j+1}}^{u_{3}+q_{2}^{(3)}} \\
& +\sum C_{3 ; i \rightarrow j} C_{3 ; j \rightarrow j+1} x_{e ; t_{j+1}}^{w_{e}^{(3)}} x_{1 ; t_{j+1}}^{p_{3}^{(3)}} x_{f ; t_{j+1}}^{u_{3}+q_{3}^{(3)}} .
\end{aligned}
$$

So by induction we have an expression of $x_{e_{i, 1} ; t_{i}}^{p} x_{e_{i, 2} ; t_{i}}^{q}$ with respect to the cluster $t_{j+1}$, that is,

$$
x_{1 ; t_{i}}^{p} x_{e_{i+1} ; t_{i}}^{q}=(35)+(37)+(39) .
$$

This expression allows us to compute the new coefficients $C_{2 ; i \rightarrow j+1}$ and $C_{3 ; i \rightarrow j+1}$ by collecting terms with nonnegative exponents on $x_{f ; t_{j+1}}$; we have

$$
C_{2 ; i \rightarrow j+1}=\sum C_{1 ; i \rightarrow j} \overline{\overline{C_{2 ; j \rightarrow j+1}}}+\sum C_{2 ; i \rightarrow j} \overline{C_{2 ; j \rightarrow j+1}}+\sum C_{3 ; i \rightarrow j} C_{2 ; j \rightarrow j+1}
$$

where the three sums are over all possible variables satisfying $u_{1}+q_{2}^{(1)} \geq 0$, $u_{2}+q_{2}^{(2)} \geq 0$, and $u_{3}+q_{2}^{(3)} \geq 0$ respectively, so that the exponent of $x_{f ; t_{j+1}}$ is nonnegative.

Similarly,

$$
C_{3 ; i \rightarrow j+1}=\sum C_{1 ; i \rightarrow j} \overline{\overline{C_{3 ; j \rightarrow j+1}}}+\sum C_{2 ; i \rightarrow j} \overline{C_{3 ; j \rightarrow j+1}}+\sum C_{3 ; i \rightarrow j} C_{3 ; j \rightarrow j+1},
$$

where the three sums are over all possible variables satisfying $u_{1}+q_{3}^{(1)} \geq 0$, $u_{2}+q_{3}^{(2)} \geq 0$, and $u_{3}+q_{3}^{(3)} \geq 0$ respectively. In particular, this shows that $C_{2 ; i \rightarrow j+1}$ and $C_{3 ; i \rightarrow j+1}$ are nonnegative integers.

Since $u_{1}, u_{2}$ or $u_{3}$ can be negative, $x_{f ; t_{j+1}}$ can have negative exponents. Now we analyze the terms in which $x_{f ; t_{j+1}}$ appears with a negative exponent. For every 
positive integer $\theta$, let $\mathcal{P}_{\theta}$ be the sum of all terms in (40) with exponent of $x_{f, t_{j+1}}$ equal to $-\theta$, that is, $\mathcal{P}_{\theta}=\mathcal{P}_{\theta, 2}+\mathcal{P}_{\theta, 3}$, where

$$
\begin{aligned}
\mathcal{P}_{\theta, 2}= & \sum C_{1 ; i \rightarrow j} \overline{\overline{C_{2 ; j \rightarrow j+1}}} x_{e ; t_{j+1}}^{v_{x_{1 ; t_{j+1}}^{(1)}}} p_{2}^{(1)} x_{f ; t_{j+1}}^{u_{1}+q_{2}^{(1)}} \\
& +\sum C_{2 ; i \rightarrow j} \overline{C_{2 ; j \rightarrow j+1}} x_{e ; t_{j+1}}^{v_{e}^{(2)}} \widetilde{x_{1 ; t_{j+1}}} p_{2}^{(2)} x_{f ; t_{j+1}}^{u_{2}+q_{2}^{(2)}} \\
& +\sum C_{3 ; i \rightarrow j} C_{2 ; j \rightarrow j+1} x_{e: t_{j+1}}^{v_{(3)}^{(3)}} \widetilde{x_{1 ; t_{j+1}}^{(3)}} x_{f ; t_{j+1}}^{u_{3}+q_{2}^{(3)}}, \\
\mathcal{P}_{\theta, 3}= & \sum C_{1 ; i \rightarrow j} \overline{\overline{C_{3 ; j \rightarrow j+1}}} x_{e ; t_{j+1}}^{w_{e}^{(1)}} x_{1 ; t_{j+1}}^{p_{3}^{(1)}} x_{f ; t_{j+1}}^{u_{1}+q_{3}^{(1)}} \\
& +\sum C_{2 ; i \rightarrow j} \overline{C_{3 ; j \rightarrow j+1}} x_{e ; t_{j+1}}^{w_{e}^{(2)}} x_{1 ; t_{j+1}}^{p_{3}^{(2)}} x_{f ; t_{j+1}}^{u_{2}+q_{3}^{(2)}} \\
& +\sum C_{3 ; i \rightarrow j} C_{3 ; j \rightarrow j+1} x_{e ; t_{j+1}}^{w_{e}^{(3)}} x_{1 ; t_{j+1}}^{p_{3}^{(3)}} x_{f ; t_{j+1}}^{u_{3}+q_{3}^{(3)}},
\end{aligned}
$$

where the first sum in the expression for $\mathcal{P}_{\theta, h}(h=2,3)$ is over all

$$
u_{1}, v_{e}^{(1)}, w_{e}^{(1)} \in \mathbb{Z}, p_{1}, q_{1}, p_{h}^{(1)}, q_{h}^{(1)} \geq 0 \quad \text { satisfying } \quad u_{1}+q_{h}^{(1)}=-\theta,
$$

the second sum is over all

$$
u_{2}, v_{e}^{(2)}, w_{e}^{(2)} \in \mathbb{Z}, p_{2}, q_{2}, p_{h}^{(2)}, q_{h}^{(2)} \geq 0 \quad \text { satisfying } \quad u_{2}+q_{h}^{(2)}=-\theta,
$$

and the third sum is over all

$$
u_{3}, v_{e}^{(3)}, w_{e}^{(3)} \in \mathbb{Z}, p_{3}, q_{3}, p_{h}^{(3)}, q_{h}^{(3)} \geq 0 \quad \text { satisfying } \quad u_{3}+q_{h}^{(3)}=-\theta .
$$

To complete the proof, we shall compute $\mathcal{P}_{\theta, 2}$ and $\mathcal{P}_{\theta, 3}$ separately. We show that $\mathcal{P}_{\theta, 3}=0$ in Lemma 4.10, and thus to complete the proof it suffices to show the following result on $\mathcal{P}_{\theta, 2}$.

Lemma 4.4. $\mathcal{P}_{\theta, 2}$ is of the form

$$
\widetilde{\widetilde{x_{f ; t_{j+1}}}} \sum_{u_{e} \in \mathbb{Z}, p_{1} \geq 0} C_{1 ; i \rightarrow j+1} x_{e ; t_{j+1}}^{u_{e}} \widetilde{x_{1 ; t_{j+1}}} p_{1},
$$

where

$$
C_{1 ; i \rightarrow j+1}=C_{1}\left(B_{t_{j+1}}, V_{k_{i}}, \ldots, V_{k_{j+1}-1}, p, q ; u_{e}, p_{1}, \theta\right)
$$

are nonnegative integers.

Proof. $P_{\theta}$ is the sum of all terms in the Laurent expansion of $x_{1 ; t_{i}}^{p} x_{f ; t_{i}}^{q}$ in the cluster $\mathbf{x}_{t_{j+1}}$ with exponent of $x_{f ; t_{j+1}}$ equal to $-\theta$. Clearly $P_{\theta, 2}=0$, for $j=i$. We shall start over and compute $P_{\theta}$ using Corollary 3.20. To keep the notation simple, we give a detailed proof for $j=i+1$. The case $j>i+1$ uses the same argument. 
Let

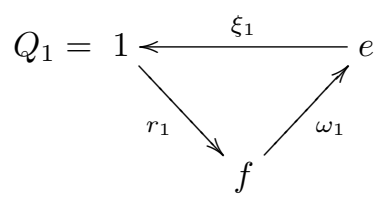

be the quiver at the seed $\mu_{1}\left(t_{i+1}\right)$, where $r_{1}, \omega_{1}$ and $\xi_{1}$ are the numbers of arrows.

Applying Corollary 3.20 to $x_{1 ; t_{i}}^{p} x_{f ; t_{i}}^{q}$, with $x_{2}=x_{f ; t_{i+1}}, x_{3}=\widetilde{x_{1 ; t_{i+1}}}$, and $z_{3}=x_{e ; t_{i+1}}$, we obtain

$$
\begin{aligned}
& \sum_{\tau_{0 ; 1}, \tau_{1 ; 1}, \ldots, \tau_{n_{1}-3 ; 1}}\left(\prod_{w=0}^{n_{1}-3}\left[\begin{array}{c}
A_{w+1 ; 1}-r_{1} s_{w ; 1} \\
\tau_{w ; 1}
\end{array}\right]\right) \\
& \times \widetilde{x_{1 ; t_{i+1}}} A_{n_{1}-1 ; 1}-r_{1} s_{n_{1}-2 ; 1} x_{f ; t_{i+1}}^{r_{1} s_{n_{1}-3 ; 1}-A_{n_{1}-2 ; 1}} x_{e ; t_{i+1}}^{\omega_{1} s_{n_{1}-2 ; 1}-\xi_{1} s_{n_{1}-3 ; 1}} .
\end{aligned}
$$

Let $n_{2}$ be the number of seeds between $\mu_{1}\left(t_{i+1}\right)$ and $t_{i+2}$ inclusive. Suppose that $n_{2}$ is an even integer. The case of $n_{2}$ odd is similar, except that the roles of $x_{1 ; t_{i}+1}$ and $x_{e ; t+1}$ are interchanged. Let

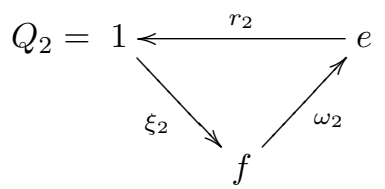

be the quiver at the seed $\mu_{1}\left(t_{i+2}\right)$, where $r_{2}, \omega_{2}$ and $\xi_{2}$ are the numbers of arrows. Since the mutation sequence relating the quivers $Q_{1}$ and $Q_{2}$ consists of mutations at the vertices 1 and $e$, we see from Lemma 4.1 that

$$
\begin{aligned}
& r_{2}=\xi_{1}, \\
& \omega_{2}=c_{n_{2}-1}^{\left[r_{2}\right]} r_{1}-c_{n_{2}-2}^{\left[r_{2}\right]} \omega_{1}, \\
& \xi_{2}=c_{n_{2}}^{\left[r_{2}\right]} r_{1}-c_{n_{2}-1}^{\left[r_{2}\right]} \omega_{1} .
\end{aligned}
$$

Now let $p_{2}=A_{n_{1}-1 ; 1}-r_{1} s_{n_{1}-2 ; 1}, q_{2}=\omega_{1} s_{n_{1}-2 ; 1}-\xi_{1} s_{n_{1}-3 ; 1}$ be the exponents of $\widetilde{x_{1 ; t_{i+1}}}$ and $x_{e ; t_{i+1}}$ in (41) respectively. Applying Corollary 3.20 to $\widetilde{x_{1 ; t_{i+1}}} p_{2} x_{e ; t_{i+1}}^{q_{2}}$ we see that (41) is equal to

$$
\begin{aligned}
& \sum_{\tau_{0 ; 1}, \tau_{1 ; 1}, \ldots, \tau_{n_{1}-3 ; 1}}\left(\prod_{w=0}^{n_{1}-3}\left[\begin{array}{c}
A_{w+1 ; 1}-r_{1} s_{w ; 1} \\
\tau_{w ; 1}
\end{array}\right]\right) x_{f ; t_{i+2}}^{r_{1} s_{n_{1}-3 ; 1}-A_{n_{1}-2 ; 1}} \\
& \times \sum_{\tau_{0 ; 2}, \tau_{1 ; 2}, \ldots, \tau_{n_{2}-3 ; 2}}\left(\prod_{w=0}^{n_{2}-3}\left[\begin{array}{c}
A_{w+1 ; 2}-r_{2} s_{w ; 2} \\
\tau_{w ; 2}
\end{array}\right]\right) \\
& \times \widetilde{x_{1 ; t_{i+2}}} A_{n_{2}-1 ; 2}-r_{2} s_{n_{2}-2 ; 2} x_{e ; t_{i+2}}^{r_{2} s_{n_{2}-3 ; 2}-A_{n_{2}-2 ; 2}} x_{f ; t_{i+2}}^{\omega_{2} s_{n_{2}-2 ; 2}-\xi_{2} s_{n_{2}-3 ; 2}}
\end{aligned}
$$




$$
\begin{aligned}
& =\sum_{\tau_{0 ; 1}, \tau_{1 ; 1}, \ldots, \tau_{n_{1}-3 ; 1}}\left(\prod_{w=0}^{n_{1}-3}\left[\begin{array}{c}
A_{w+1 ; 1}-r_{1} s_{w ; 1} \\
\tau_{w ; 1}
\end{array}\right]\right) \\
& \times \sum_{\tau_{0 ; 2}, \tau_{1 ; 2}, \ldots, \tau_{n_{2}-3 ; 2}}\left(\prod_{w=0}^{n_{2}-3}\left[\begin{array}{c}
A_{w+1 ; 2}-r_{2} s_{w ; 2} \\
\tau_{w ; 2}
\end{array}\right]\right) \\
& \times \widetilde{x_{1 ; t_{i+2}}} A_{n_{2}-1 ; 2}-r_{2} s_{n_{2}-2 ; 2} x_{e ; t_{i+2}}^{r_{2} s_{n_{2}-3 ; 2}-A_{n_{2}-2 ; 2}} x_{f ; t_{i+2}}^{\omega_{2} s_{n_{2}-2 ; 2}-\xi_{2} s_{n_{2}-3 ; 2}+r_{1} s_{n_{1}-3 ; 1}-A_{n_{1}-2 ; 1}}
\end{aligned}
$$

where $A_{i ; 2}$ and $s_{i ; 2}$ are as defined before Lemma 3.12 and Lemma 3.15 but in terms of $p_{2}, q_{2}$, and $r_{2}$, thus $A_{i ; 2}=p_{2} c_{i+1}^{\left[r_{2}\right]}+q_{2} c_{i}^{\left[r_{2}\right]}$ and $s_{i ; 2}=\sum_{j=0}^{i-1} c_{i-j+1}^{\left[r_{2}\right]} \tau_{j ; 2}$.

Let $\theta$ be a positive integer. We want to compute $P_{\theta}$, which is the sum of all terms in the sum above for which the exponent of $x_{f ; t_{i+2}}$ is equal to $-\theta$, and show that it is divisible by $\widetilde{\widetilde{x_{f ; t_{j+1}}}} \theta$. Thus $-\theta$ is equal to

$$
\omega_{2} s_{n_{2}-2 ; 2}-\xi_{2} s_{n_{2}-3 ; 2}+r_{1} s_{n_{1}-3 ; 1}-A_{n_{1}-2 ; 1} .
$$

It is convenient to introduce $\varsigma$ such that $\tau_{0 ; 2}=\varsigma-s_{n_{1}-3 ; 1}$. Then

$$
\begin{aligned}
& s_{n_{2}-2 ; 2}=c_{n_{2}-1}^{\left[r_{2}\right]}\left(\varsigma-s_{n_{1}-3 ; 1}\right)+\sum_{j=1}^{n_{2}-3} c_{n_{2}-1-j}^{\left[r_{2}\right]} \tau_{j ; 2}, \\
& s_{n_{2}-3 ; 2}=c_{n_{2}-2}^{\left[r_{2}\right]}\left(\varsigma-s_{n_{1}-3 ; 1}\right)+\sum_{j=1}^{n_{2}-4} c_{n_{2}-2-j}^{\left[r_{2}\right]} \tau_{j ; 2} .
\end{aligned}
$$

Using (42), the expressions for $s_{n_{2}-2 ; 2}$ and $s_{n_{2}-3 ; 2}$ and the fact that $c_{1}^{[\xi]}=0$, we have

$$
\begin{aligned}
& \omega_{2} s_{n_{2}-2 ; 2}-\xi_{2} s_{n_{2}-3 ; 2} \\
& =\left(c_{n_{2}-1}^{\left[\xi_{1}\right]} r_{1}-c_{n_{2}-2}^{\left[\xi_{1}\right]} \omega_{1}\right)\left[c_{n_{2}-1}^{\left[\xi_{1}\right]}\left(\varsigma-s_{n_{1}-3 ; 1}\right)+\sum_{j=1}^{n_{2}-3} c_{n_{2}-1-j}^{\left[\xi_{1}\right]} \tau_{j ; 2}\right] \\
& \quad-\left(c_{n_{2}}^{\left[\xi_{1}\right]} r_{1}-c_{n_{2}-1}^{\left[\xi_{1}\right]} \omega_{1}\right)\left[c_{n_{2}-2}^{\left[\xi_{1}\right]}\left(\varsigma-s_{n_{1}-3 ; 1}\right)+\left(\sum_{j=1}^{n_{2}-3} c_{n_{2}-2-j}^{\left[\xi_{1}\right]} \tau_{j ; 2}\right)-c_{1}^{\left[\xi_{1}\right]} \tau_{n_{2}-3 ; 2}\right] \\
& =\left(\varsigma-s_{n_{1}-3 ; 1}\right) r_{1}\left(\left(c_{n_{2}-1}^{\left[\xi_{1}\right]}\right)^{2}-c_{n_{2}}^{\left[\xi_{1}\right]} c_{n_{2}-2}^{\left[\xi_{1}\right]}\right) \\
& +\sum_{j=1}^{n_{2}-3} \tau_{j ; 2}\left[r_{1}\left(c_{n_{2}-1}^{\left[\xi_{1}\right]} c_{n_{2}-1-j}^{\left[\xi_{1}\right]}-c_{n_{2}}^{\left[\xi_{1}\right]} c_{n_{2}-2-j}^{\left[\xi_{1}\right]}\right)+\omega_{1}\left(-c_{n_{2}-2}^{\left[\xi_{1}\right]} c_{n_{2}-1-j}^{\left[\xi_{1}\right]}+c_{n_{2}-1}^{\left[\xi_{1}\right]} c_{n_{2}-2-j}^{\left[\xi_{1}\right]}\right)\right] \\
& \stackrel{3.1}{=}\left(\varsigma-s_{n_{1}-3 ; 1}\right) r_{1}+\sum_{j=1}^{n_{2}-3} \tau_{j ; 2}\left[r_{1}\left(-c_{-j}^{\left[\xi_{1}\right]}\right)+\omega_{1} c_{1-j}^{\left[\xi_{1}\right]}\right] .
\end{aligned}
$$


And since $-c_{-j}^{\left[\xi_{1}\right]}=c_{j+2}^{\left[\xi_{1}\right]}$, we get

$$
\begin{aligned}
-\theta & =r_{1}\left(\varsigma-s_{n_{1}-3 ; 1}\right)+\sum_{j=1}^{n_{2}-3} \tau_{j ; 2}\left(c_{j+2}^{\left[\xi_{1}\right]} r_{1}-c_{j+1}^{\left[\xi_{1}\right]} \omega_{1}\right)+r_{1} s_{n_{1}-3 ; 1}-A_{n_{1}-2 ; 1} \\
& =-A_{n_{1}-2 ; 1}+r_{1} \varsigma+\sum_{j=1}^{n_{2}-3} \tau_{j ; 2}\left(c_{j+2}^{\left[\xi_{1}\right]} r_{1}-c_{j+1}^{\left[\xi_{1}\right]} \omega_{1}\right) .
\end{aligned}
$$

Also, the exponents of $\widetilde{x_{1 ; t_{i+2}}}$ and $x_{e ; t_{i+2}}$ in (43) can be expressed as follows:

$$
\begin{aligned}
A_{n_{2}-1 ; 2}-r_{2} s_{n_{2}-2 ; 2}= & c_{n_{2}}^{\left[\xi_{1}\right]} p_{2}+c_{n_{2}-1}^{\left[\xi_{1}\right]} q_{2}-\xi_{1}\left(c_{n_{2}-1}^{\left[\xi_{1}\right]}\left(\varsigma-s_{n_{1}-3 ; 1}\right)+\sum_{j=1}^{n_{2}-3} c_{n_{2}-1-j}^{\left[\xi_{1}\right]} \tau_{j ; 2}\right) \\
= & c_{n_{2}}^{\left[\xi_{1}\right]}\left(A_{n_{1}-1 ; 1}-r_{1} s_{n_{1}-2 ; 1}\right)+c_{n_{2}-1}^{\left[\xi_{1}\right]}\left(\omega_{1} s_{n_{1}-2 ; 1}-\xi_{1} s_{n_{1}-3 ; 1}\right) \\
& -\xi_{1}\left(c_{n_{2}-1}^{\left[\xi_{1}\right]}\left(\varsigma-s_{n_{1}-3 ; 1}\right)+\sum_{j=1}^{n_{2}-3} c_{n_{2}-1-j}^{\left[\xi_{1}\right]} \tau_{j ; 2}\right) \\
= & c_{n_{2}}^{\left[\xi_{1}\right]}\left(A_{n_{1}-1 ; 1}-r_{1} s_{n_{1}-2 ; 1}\right)+c_{n_{2}-1}^{\left[\xi_{1}\right]} \omega_{1} s_{n_{1}-2 ; 1} \\
& -\xi_{1}\left(c_{n_{2}-1}^{\left[\xi_{1}\right]} \varsigma+\sum_{j=1}^{n_{2}-3} c_{n_{2}-1-j}^{\left[\xi_{1}\right]} \tau_{j ; 2}\right),
\end{aligned}
$$

and similarly

$$
\begin{aligned}
\xi_{1} s_{n_{2}-3 ; 2}-A_{n_{2}-2 ; 2}= & \xi_{1}\left(c_{n_{2}-2}^{\left[\xi_{1}\right]} \varsigma+\sum_{j=1}^{n_{2}-4} c_{n_{2}-2-j}^{\left[\xi_{1}\right]} \tau_{j ; 2}\right) \\
& -\left(c_{n_{2}-1}^{\left[\xi_{1}\right]}\left(A_{n_{1}-1 ; 1}-r_{1} s_{n_{1}-2 ; 1}\right)+c_{n_{2}-2}^{\left[\xi_{1}\right]} \omega_{1} s_{n_{1}-2 ; 1}\right)
\end{aligned}
$$

By fixing $\varsigma, \tau_{1 ; 2}, \ldots, \tau_{n_{2}-3 ; 2}$ in $(43)$, we have

$$
\begin{aligned}
\sum_{\tau_{0 ; 1}, \tau_{1 ; 1}, \ldots, \tau_{n_{1}-3 ; 1}} & \left(\prod_{w=0}^{n_{1}-3}\left[\begin{array}{c}
A_{w+1 ; 1}-r_{1} s_{w ; 1} \\
\tau_{w ; 1}
\end{array}\right]\right)\left(\prod_{w=0}^{n_{2}-3}\left[\begin{array}{c}
A_{w+1 ; 2}-r_{2} s_{w ; 2} \\
\tau_{w ; 2}
\end{array}\right]\right) \\
& \times \widetilde{x_{1 ; t_{i+2}}} c_{n_{2}}^{\left[\xi_{1}\right]}\left(A_{n_{1}-1 ; 1}-r_{1} s_{n_{1}-2 ; 1}\right)+c_{n_{2}-1}^{\left[\xi_{1}\right]} \omega_{1} s_{n_{1}-2 ; 1}-\xi_{1}\left(c_{n_{2}-1}^{\left[\xi_{1}\right]} \varsigma+\sum_{j=1}^{n_{2}-3} c_{n_{2}-1-j}^{\left[\xi_{1}\right]} \tau_{j ; 2}\right) \\
& \times x_{e ; t_{i+2}}^{\xi_{1}\left(c_{n_{2}-2}^{\left[\xi_{1}\right]} \varsigma+\sum_{j=1}^{n_{2}-4} c_{n_{2}-2-j}^{\left[\xi_{1}\right]} \tau_{j ; 2}\right)-\left(c_{n_{2}-1}^{\left[\xi_{1}\right]}\left(A_{n_{1}-1 ; 1}-r_{1} s_{n_{1}-2 ; 1}\right)+c_{n_{2}-2}^{\left[\xi_{1}\right]} \omega_{1} s_{n_{1}-2 ; 1}\right)} \\
& \times x_{f ; t_{i+2}}^{-A_{n_{1}-2 ; 1}+r_{1} \varsigma+\sum_{j=1}^{n_{2}-3}\left(c_{j+2}^{\left[t_{1}\right]} r_{1}-c_{j+1}^{\left[t_{1}\right]} \omega_{1}\right) \tau_{j ; 2}}
\end{aligned}
$$

and this is equal to a product $\phi \varphi$ where $\phi$ is a Laurent monomial in $\widetilde{x_{1 ; t_{i+2}}}, x_{e ; t_{i+2}}$, 
$x_{f ; t_{i+2}}$, while $\varphi$ is equal to

$$
\begin{aligned}
& \sum_{\tau_{0 ; 1}, \tau_{1 ; 1}, \ldots, \tau_{n_{1}-3 ; 1}}\left(\prod_{w=0}^{n_{1}-3}\left[\begin{array}{c}
A_{w+1 ; 1}-r_{1} s_{w ; 1} \\
\tau_{w ; 1}
\end{array}\right]\right)\left(\prod_{w=0}^{n_{2}-3}\left[\begin{array}{c}
A_{w+1 ; 2}-r_{2} s_{w ; 2} \\
\tau_{w ; 2}
\end{array}\right]\right) \\
& \times\left(\frac{\widetilde{x_{1 ; t_{i+2}}} c_{n_{2}}^{\left[\xi_{1}\right]} r_{1}-c_{n_{2}-1}^{\left[\xi_{1}\right]} \omega_{1}}{x_{e ; t_{i+2}}^{c_{n_{2}-1}^{\left[\xi_{1}\right]} r_{1}-c_{n_{2}-2}^{\left[\xi_{1}\right]} \omega_{1}}}\right)^{\left\lfloor\left(A_{n_{1}-1 ; 1}-\varsigma\right) \frac{A_{n_{1}-1 ; 1}}{A_{n_{1} ; 1}}\right\rfloor-s_{n_{1}-2 ; 1}}
\end{aligned}
$$

and transferring the 0 -th term of the second product to an $\left(n_{1}-2\right)$-nd term in the first product, we get

$$
\begin{aligned}
& \sum_{\tau_{0 ; 1}, \tau_{1 ; 1}, \ldots, \tau_{n_{1}-2 ; 1}}\left(\prod_{w=0}^{n_{1}-2}\left[\begin{array}{c}
A_{w+1 ; 1}-r_{1} s_{w ; 1} \\
\tau_{w ; 1}
\end{array}\right]\right)\left(\prod_{w=1}^{n_{2}-3}\left(\begin{array}{c}
A_{w+1 ; 2}-r_{2} s_{w ; 2} \\
\tau_{w ; 2}
\end{array}\right)\right) \\
& \times\left(\frac{\widetilde{x_{1 ; t_{i+2}}} c_{n_{2}}^{\left[\xi_{1}\right]} r_{1}-c_{n_{2}-1}^{\left[\xi_{1}\right]} \omega_{1}}{x_{n_{2}-1}^{\left[\xi_{1}\right]} r_{1}-c_{n_{2}-2}^{\left[\xi_{1}\right]} \omega_{1}}\right)^{\left\lfloor\left(A_{n_{1}-1 ; 1}-\varsigma\right) \frac{A_{n_{1}-1 ; 1}}{A_{n_{1} ; 1}}\right\rfloor-s_{n_{1}-2 ; 1}},
\end{aligned}
$$

where $\tau_{n_{1}-2 ; 1}=A_{n_{1}-1 ; 1}-r_{1} s_{n_{1}-2 ; 1}-\tau_{0 ; 2}=A_{n_{1}-1 ; 1}-r_{1} s_{n_{1}-2 ; 1}-\varsigma+s_{n_{1}-3 ; 1}$. Moreover, using Lemma 3.15, we observe that

$$
s_{n_{1}-1 ; 1}=r_{1} s_{n_{1}-2 ; 1}-s_{n_{1}-3 ; 1}+\tau_{n_{1}-2 ; 1}=A_{n_{1}-1 ; 1}-\varsigma,
$$

and by Theorem 3.26,

$$
\sum_{\substack{\tau_{0 ; 1}, \tau_{1 ; 1}, \ldots, \tau_{n_{1}-2 ; 1} \\
s_{n_{1}-1 ; 1}=A_{n_{1}-1 ; 1}-\varsigma}}\left(\prod_{w=0}^{n_{1}-2}\left[\begin{array}{c}
A_{w+1 ; 1}-r_{1} s_{w ; 1} \\
\tau_{w ; 1}
\end{array}\right]\right) x_{1 ; t_{i+1}}^{A_{n_{1}-1 ; 1}-r_{1} s_{n_{1}-2 ; 1}}
$$

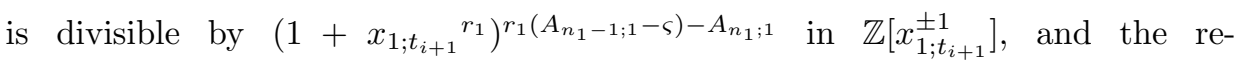
sulting quotient has nonnegative coefficients. Multiplying the sum with $r_{1: t_{i+1}}\left\lfloor\left(A_{n_{1}-1 ; 1}-\varsigma\right) \frac{A_{n_{1}-1 ; 1}}{A_{n_{1} ; 1}}\right\rfloor-A_{n_{1}-1 ; 1}$ shows that

$$
\sum_{\substack{\tau_{0 ; 1}, \tau_{1 ; 1}, \ldots, \tau_{n_{1}-2 ; 1} \\
s_{n_{1}-1 ; 1}=A_{n_{1}-1 ; 1}-\varsigma}}\left(\prod_{w=0}^{n_{1}-2}\left[\begin{array}{c}
A_{w+1 ; 1}-r_{1} s_{w ; 1} \\
\tau_{w ; 1}
\end{array}\right]\right)\left(x_{1 ; t_{i+1}} r_{1}\right)^{\left\lfloor\left(A_{n_{1}-1 ; 1}-\varsigma\right) \frac{A_{n_{1}-1 ; 1}}{A_{n_{1} ; 1}}\right\rfloor-s_{n_{1}-2 ; 1}}
$$

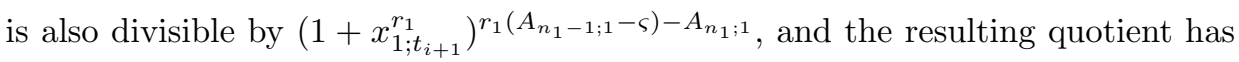
nonnegative coefficients. Moreover, we shall show in Lemma 4.5 below that the exponents in (47) are nonnegative, which implies that the quotient is a polynomial. 
Note that the statement about the divisibility of (47) also holds when we replace $x_{1 ; t_{i+1}}^{r}$ with any other expression $X$. We can write (45) as follows:

$$
(45)=\sum \sum_{m} q(m) p(m) X^{b-m},
$$

where

$$
\begin{aligned}
& q(m)=\prod_{w=0}^{n_{1}-2}\left[\begin{array}{c}
A_{w+1 ; 1}-r_{1} s_{w ; 1} \\
\tau_{w ; 1}
\end{array}\right], \quad p(m)=\prod_{w=1}^{n_{2}-3}\left(\begin{array}{c}
A_{w+1 ; 2}-r_{2} s_{w ; 2} \\
\tau_{w ; 2}
\end{array}\right), \\
& b=\left\lfloor\left(A_{n_{1}-1 ; 1}-\varsigma\right) \frac{A_{n_{1}-1 ; 1}}{A_{n_{1} ; 1}}\right\rfloor, \quad m=s_{n_{1}-2 ; 1}, \\
& X=\frac{\widetilde{x_{1 ; t_{i+2}}} c_{n_{2}}^{\left[\xi_{1}\right]} r_{1}-c_{n_{2}-1}^{\left[\xi_{1}\right]} \omega_{1}}{x_{e ; t_{i+2}}^{c_{n_{2}-1}^{\left[\xi_{1}\right]} r_{1}-c_{n_{2}-2}^{\left[\xi_{1}\right]} \omega_{1}}} .
\end{aligned}
$$

Then Lemma 4.7 below yields

$$
p(m)=\sum_{i=0}^{\sum_{w=1}^{n_{2}-3} \tau_{w ; 2}} d_{i}\left(\begin{array}{c}
\left\lfloor\left(A_{n_{1}-1 ; 1}-\varsigma\right) \frac{A_{n_{1}-1 ; 1}}{A_{n_{1} ; 1}}\right\rfloor-s_{n_{1}-2 ; 1} \\
i
\end{array}\right)
$$

and using Lemma 4.6 with $g=r_{1}\left(A_{n_{1}-1 ; 1}-\varsigma\right)-A_{n_{1} ; 1}$ and $h=\sum_{j=1}^{n_{2}-3} \tau_{j ; 2}$, we find that the expression in (45) is divisible by

$$
\begin{aligned}
\left(1+\frac{\widetilde{x_{1 ; t_{i+2}}} c_{n_{2}}^{\left[\xi_{1}\right]} r_{1}-c_{n_{2}-1}^{\left[\xi_{1}\right]} \omega_{1}}{x_{x_{n_{2}-1} r_{i+2}}^{\left.c_{1}\right]} r_{1}-c_{n_{2}-2}^{\left[\xi_{1}\right]} \omega_{1}}\right)^{r_{1}\left(A_{n_{1}-1 ; 1}-\varsigma\right)-A_{n_{1} ; 1}-\sum_{j=1}^{n_{2}-3} \tau_{j ; 2}} \\
=\left(1+\frac{\widetilde{x_{1 ; t_{i+2}}}}{x_{e ; t_{i+2}}^{\omega_{2}}}\right)^{A_{n_{1}-2 ; 1}-r_{1} \varsigma-\sum_{j=1}^{n_{2}-3} \tau_{j ; 2}}
\end{aligned}
$$

and the resulting quotient has nonnegative coefficients. Finally, dividing (48) by $x_{f ; t_{i+2}}^{\theta}$ and using the fact that

we see that $\mathcal{P}_{\theta, 2}$ is divisible by $\widetilde{\widetilde{x_{f ; t_{i+2}}}}$.

$$
\widetilde{\widetilde{x_{f ; t_{i+2}}}}=\left(x_{e ; t_{i+2}}^{\omega_{2}}+\widetilde{x_{1 ; t_{i+2}}} \xi_{2}\right) / x_{f ; t_{i+2}}
$$

\section{Lemma 4.5.}

$$
\left\lfloor\left(A_{n_{1}-1 ; 1}-\varsigma\right) \frac{A_{n_{1}-1 ; 1}}{A_{n_{1} ; 1}}\right\rfloor-s_{n_{1}-2 ; 1} \geq 0
$$

Proof. We have

$$
s_{n_{1}-1 ; 1}-s_{n_{1}-1 ; 1}^{\prime} \geq\left(s_{n_{1}-2 ; 1}-s_{n_{1}-2 ; 1}^{\prime}\right) \frac{A_{n_{1}-1 ; 1}}{A_{n_{1}-2 ; 1}} \geq\left(s_{n_{1}-2 ; 1}-s_{n_{1}-2 ; 1}^{\prime}\right) \frac{A_{n_{1} ; 1}}{A_{n_{1}-1 ; 1}},
$$

where the first inequality follows from (16) and the second from Lemma 3.12(b). 
Hence

$$
\left(s_{n_{1}-1 ; 1}-s_{n_{1}-1 ; 1}^{\prime}\right) A_{n_{1}-1 ; 1} \geq\left(s_{n_{1}-2 ; 1}-s_{n_{1}-2 ; 1}^{\prime}\right) A_{n_{1} ; 1} .
$$

On the other hand, since $s_{n_{1}-2 ; 1}^{\prime}=k c_{n_{1}-1 ; 1}^{\left[r_{1}\right]}, s_{n_{1}-1 ; 1}^{\prime}=k c_{n ; 1}^{\left[r_{1}\right]}$, by Definition 3.16 , and $A_{i ; 1}=p c_{i+1 ; 1}^{\left[r_{1}\right]}+q c_{i ; 1}^{\left[r_{1}\right]}$, we have

$$
\begin{aligned}
s_{n_{1}-1 ; 1}^{\prime} A_{n_{1}-1 ; 1} & =k c_{n ; 1}^{\left[r_{1}\right]}\left(p c_{n ; 1}^{\left[r_{1}\right]}+q c_{n_{1}-1 ; 1}^{\left[r_{1}\right]}\right) \\
& \geq k c_{n_{1}-1 ; 1}^{\left[r_{1}\right]}\left(p c_{n+1 ; 1}^{\left[r_{1}\right]}+q c_{n ; 1}^{\left[r_{1}\right]}\right)=s_{n_{1}-2 ; 1}^{\prime} A_{n_{1} ; 1},
\end{aligned}
$$

where the inequality follows from Lemma 3.1. Adding (49) and (50) we get

$$
s_{n_{1}-1 ; 1} A_{n_{1}-1 ; 1} \geq s_{n_{1}-2 ; 1} A_{n_{1} ; 1} .
$$

Therefore (46) yields

$$
\left(A_{n_{1}-1 ; 1}-\varsigma\right) A_{n_{1}-1 ; 1} \geq s_{n_{1}-2 ; 1} A_{n_{1} ; 1},
$$

and we get

$$
\left(A_{n_{1}-1 ; 1}-\varsigma\right) \frac{A_{n_{1}-1 ; 1}}{A_{n_{1} ; 1}}-s_{n_{1}-2 ; 1} \geq s_{n_{1}-2 ; 1}-s_{n_{1}-2 ; 1}=0 .
$$

Lemma 4.6. Suppose that a polynomial in $x$ of the form

$$
\sum_{m \in I} q(m) x^{b-m}
$$

is divisible by $(1+x)^{g}$ and the quotient has nonnegative coefficients. Let

$$
p(m)=\sum_{i=0}^{h} d_{i}\left(\begin{array}{c}
b-m \\
i
\end{array}\right)
$$

be a polynomial in $m$ with $d_{i} \geq 0$. Then

$$
\sum_{m \in I} p(m) q(m) x^{b-m}
$$

is divisible by $(1+x)^{g-h}$ and the quotient has nonnegative coefficients.

Proof. This is because $x^{i} \frac{d^{i}}{d x^{i}} \sum_{m \in I} q(m) x^{b-m}$ is divisible by $(1+x)^{g-i}$ and the quotient has nonnegative coefficients.

Lemma 4.7. With assumptions in the proof of Lemma 4.4, we have

$$
\prod_{w=1}^{n_{2}-3}\left(\begin{array}{c}
A_{w+1 ; 2}-r_{2} s_{w ; 2} \\
\tau_{w ; 2}
\end{array}\right)=\sum_{i=0}^{\sum_{w=1}^{n_{2}-3} \tau_{w ; 2}} d_{i}\left(\begin{array}{c}
\left\lfloor\left(A_{n_{1}-1 ; 1}-\varsigma\right) \frac{A_{n_{1}-1 ; 1}}{A_{n_{1} ; 1}}\right\rfloor-s_{n_{1}-2 ; 1} \\
i
\end{array}\right)
$$

for some $d_{i} \in \mathbb{N}$, which are independent of $s_{n_{1}-2 ; 1}$. 
Proof. Once we know that there are nonnegative integers $a$ and $b$ such that $A_{w+1 ; 2}-r_{2} s_{w ; 2}=a\left(\left\lfloor\left(A_{n_{1}-1 ; 1}-\varsigma\right) \frac{A_{n_{1}-1 ; 1}}{A_{n_{1} ; 1}}\right\rfloor-s_{n_{1}-2 ; 1}\right)+b$, it is clear, by Lemma 4.8 , that

$$
\left(\begin{array}{c}
A_{w+1 ; 2}-r_{2} s_{w ; 2} \\
\tau_{w ; 2}
\end{array}\right)=\sum_{i=0}^{\tau_{w ; 2}} d_{i}^{\prime}\left(\begin{array}{c}
\left\lfloor\left(A_{n_{1}-1 ; 1}-\varsigma\right) \frac{A_{n_{1}-1 ; 1}}{A_{n_{1} ; 1}}\right\rfloor-s_{n_{1}-2 ; 1} \\
i
\end{array}\right)
$$

for some $d_{i}^{\prime} \in \mathbb{N}$, and by Lemma 4.9 , for any nonnegative integers $j$ and $k$,

$$
\begin{array}{r}
\left(\begin{array}{c}
\left.\left\lfloor\left(A_{n_{1}-1 ; 1}-\varsigma\right) \frac{\left.A_{n_{1}-1 ; 1}\right\rfloor}{A_{n_{1} ; 1}}\right\rfloor-s_{n_{1}-2 ; 1}\right) \\
j
\end{array}\right)\left(\begin{array}{c}
\left\lfloor\left(A_{n_{1}-1 ; 1}-\varsigma\right) \frac{A_{n_{1}-1 ; 1}}{A_{n_{1} ; 1}}\right\rfloor-s_{n_{1}-2 ; 1} \\
k
\end{array}\right) \\
=\sum_{i=0}^{j+k} d_{i}^{\prime \prime}\left(\begin{array}{c}
\left\lfloor\left(A_{n_{1}-1 ; 1}-\varsigma\right) \frac{A_{n_{1}-1 ; 1}}{A_{n_{1} ; 1}}\right\rfloor-s_{n_{1}-2 ; 1} \\
i
\end{array}\right)
\end{array}
$$

for some $d_{i}^{\prime \prime} \in \mathbb{N}$. Then the desired statement easily follows.

Thus we need to show the existence of the nonnegative integers $a$ and $b$. Using the definitions of $A_{w+1 ; 2}$ and $\varsigma$ as well as the fact that $r_{2}=\xi_{1}$, we get

$$
\begin{aligned}
& A_{w+1 ; 2}-r_{2} s_{w ; 2} \\
& \quad=c_{w+2}^{\left[\xi_{1}\right]}\left(A_{n_{1}-1 ; 1}-r_{1} s_{n_{1}-2 ; 1}\right)+c_{w+1}^{\left[\xi_{1}\right]} \omega_{1} s_{n_{1}-2 ; 1}-\xi_{1}\left(c_{w+1}^{\left[\xi_{1}\right]} \varsigma+\sum_{j=1}^{w-1} c_{w+1-j}^{\left[\xi_{1}\right]} \tau_{j ; 2}\right),
\end{aligned}
$$

which can be written as

$$
A_{w+1 ; 2}-r_{2} s_{w ; 2}=\left(c_{w+2}^{\left[\xi_{1}\right]} r_{1}-c_{w+1}^{\left[\xi_{1}\right]} \omega_{1}\right)\left(\left\lfloor\left(A_{n_{1}-1 ; 1}-\varsigma\right) \frac{A_{n_{1}-1 ; 1}}{A_{n_{1} ; 1}}\right\rfloor-s_{n_{1}-2 ; 1}\right)+C(w)
$$

where $C(w)$ is some function of $w$, which is independent of $s_{n_{1}-2 ; 1}$. Note that

$$
c_{w+2}^{\left[\xi_{1}\right]} r_{1}-c_{w+1}^{\left[\xi_{1}\right]} \omega_{1}>0
$$

because, by Lemma 4.1, this is the number of arrows between some pair of vertices in some seed between $t_{i+1}$ and $t_{i+2}$. Thus it suffices to show that $C(w)$ is nonnegative. Indeed,

$$
\begin{aligned}
C(w)= & \left(c_{w+2}^{\left[\xi_{1}\right]} r_{1}-c_{w+1}^{\left[\xi_{1}\right]} \omega_{1}\right)\left(\left(A_{n_{1}-1 ; 1}-\varsigma\right) \frac{A_{n_{1}-1 ; 1}}{A_{n_{1} ; 1}}-\left\lfloor\left(A_{n_{1}-1 ; 1}-\varsigma\right) \frac{A_{n_{1}-1 ; 1}}{A_{n_{1} ; 1}}\right\rfloor\right) \\
& +\tilde{C}(w) \theta(w),
\end{aligned}
$$


where

$$
\begin{aligned}
\tilde{C}(w)= & c_{w+2}^{\left[\xi_{1}\right]}-\left(c_{w+2}^{\left[\xi_{1}\right]} r_{1}-c_{w+1}^{\left[\xi_{1}\right]} \omega_{1}\right) \frac{A_{n_{1}-1 ; 1}}{A_{n_{1} ; 1}} \\
\theta(w)= & A_{n_{1}-1 ; 1}-\frac{\xi_{1} c_{w+1}^{\left[\xi_{1}\right]}-\left(c_{w+2}^{\left[\xi_{1}\right]} r_{1}-c_{w+1}^{\left[\xi_{1}\right]} \omega_{1}\right) \frac{A_{n_{1}-1 ; 1}}{A_{n_{1} ; 1}}}{c_{w+2}^{\left[\xi_{1}\right]}-\left(c_{w+2}^{\left[\xi_{1}\right]} r_{1}-c_{w+1}^{\left[\xi_{1}\right]} \omega_{1}\right) \frac{A_{n_{1}-1 ; 1}}{A_{n_{1} ; 1}}} \varsigma \\
& -\sum_{j=1}^{w-1} \frac{\xi_{1} c_{w+1-j}^{\left[\xi_{1}\right]}}{c_{w+2}^{\left[\xi_{1}\right]}-\left(c_{w+2}^{\left[\xi_{1}\right]} r_{1}-c_{w+1}^{\left[\xi_{1}\right]} \omega_{1}\right) \frac{A_{n_{1}-1 ; 1}}{A_{n_{1} ; 1}}} \tau_{j ; 2} .
\end{aligned}
$$

We want to show that $C(w)$ is nonnegative for $w \geq 1$, for which it suffices to show that $\tilde{C}(w)$ and $\theta(w)$ are nonnegative for $w \geq 1$.

First we show that $\tilde{C}(w)$ are nonnegative for $w \geq 1$. Note that $\tilde{C}(w)=$ $\xi_{1} \tilde{C}(w-1)-\tilde{C}(w-2)$. Moreover $\xi_{1} \geq 2$, by Lemma 4.1. Hence if we show $\tilde{C}(1)>0 \geq \tilde{C}(0)$ then induction on $w$ will show that $\tilde{C}(w)$ is increasing in $w$. It is easy to see that $\tilde{C}(0)=1-r_{1} \frac{A_{n_{1}-1 ; 1}}{A_{n_{1} ; 1}} \leq 0$. On the other hand,

$$
\begin{aligned}
\tilde{C}(1) & =\xi_{1}-\left(\xi_{1} r_{1}-\omega_{1}\right) \frac{A_{n_{1}-1 ; 1}}{A_{n_{1} ; 1}}=\xi_{1} \frac{A_{n_{1} ; 1}-r_{1} A_{n_{1}-1 ; 1}}{A_{n_{1} ; 1}}+\omega_{1} \frac{A_{n_{1}-1 ; 1}}{A_{n_{1} ; 1}} \\
& =\xi_{1} \frac{-A_{n_{1}-2 ; 1}}{A_{n_{1} ; 1}}+\omega_{1} \frac{A_{n_{1}-1 ; 1}}{A_{n_{1} ; 1}}
\end{aligned}
$$

which is positive because

$$
\begin{aligned}
\omega_{1} A_{n_{1}-1 ; 1}-\xi_{1} A_{n_{1}-2 ; 1} & =\omega_{1}\left(p c_{n_{1}}+q c_{n_{1}-1}\right)-\xi_{1}\left(p c_{n_{1}-1}+q c_{n_{1}-2}\right) \\
& \left.=p\left(\omega_{1} c_{n_{1}}-\xi_{1} c_{n_{1}-1}\right)+q\left(\omega_{1} c_{n_{1}-1}-\xi_{1} c_{n_{1}-2}\right)\right)>0
\end{aligned}
$$

where the last inequality follows from

$$
\begin{aligned}
\left\{\omega_{1} c_{n_{1}}-\xi_{1} c_{n_{1}-1}, \omega_{1} c_{n_{1}-1}-\xi_{1} c_{n_{1}-2}\right\} & \\
= & \left\{\text { the number of arrows between } e \text { and } 1 \text { in the seed } t_{i},\right. \\
& \text { the number of arrows between } \left.e \text { and } f \text { in the seed } t_{i}\right\} .
\end{aligned}
$$

Next we show that $\theta(w)$ is nonnegative for all $w$ such that $1 \leq w \leq n_{2}-3$. Recall from (44) that

$$
\theta=A_{n_{1}-2 ; 1}-r_{1} \varsigma-\sum_{j=1}^{n_{2}-3}\left(c_{j+2}^{\left[t_{1}\right]} r_{1}-c_{j+1}^{\left[t_{1}\right]} \omega_{1}\right) \tau_{j ; 2}>0
$$

which implies that

$$
A_{n_{1}-1 ; 1}-\frac{r_{1} A_{n_{1}-1 ; 1}}{A_{n_{1}-2 ; 1}} \varsigma-\sum_{j=1}^{w-1} \frac{\left(c_{j+2}^{\left[t_{1}\right]} r_{1}-c_{j+1}^{\left[t_{1}\right]} \omega_{1}\right) A_{n_{1}-1 ; 1}}{A_{n_{1}-2 ; 1}} \tau_{j ; 2}>0 .
$$


So it is enough to show

$$
\frac{r_{1} A_{n_{1}-1 ; 1}}{A_{n_{1}-2 ; 1}}>\frac{\xi_{1} c_{w+1}^{\left[\xi_{1}\right]}-\left(c_{w+2}^{\left[\xi_{1}\right]} r_{1}-c_{w+1}^{\left[\xi_{1}\right]} \omega_{1}\right) \frac{A_{n_{1}-1 ; 1}}{A_{n_{1} ; 1}}}{c_{w+2}^{\left[\xi_{1}\right]}-\left(c_{w+2}^{\left[\xi_{1}\right]} r_{1}-c_{w+1}^{\left[\xi_{1}\right]} \omega_{1}\right) \frac{A_{n_{1}-1 ; 1}}{A_{n_{1} ; 1}}}
$$

and

$$
\frac{\left(c_{j+2}^{\left[t_{1}\right]} r_{1}-c_{j+1}^{\left[t_{1}\right]} \omega_{1}\right) A_{n_{1}-1 ; 1}}{A_{n_{1}-2 ; 1}}>\frac{\xi_{1} c_{w+1-j}^{\left[\xi_{1}\right]}}{c_{w+2}^{\left[\xi_{1}\right]}-\left(c_{w+2}^{\left[\xi_{1}\right]} r_{1}-c_{w+1}^{\left[\xi_{1}\right]} \omega_{1}\right) \frac{A_{n_{1}-1 ; 1}}{A_{n_{1} ; 1}}} ;
$$

but these inequalities can be proved by induction on $w$.

Lemma 4.8. Let $a, b, c$ be any nonnegative integers. Then there are nonnegative integers $d_{0}, \ldots, d_{c}$ such that

$$
\left(\begin{array}{c}
a X+b \\
c
\end{array}\right)=\sum_{i=0}^{c} d_{i}\left(\begin{array}{c}
X \\
i
\end{array}\right) \text { for all nonnegative integers } X .
$$

Proof. The Vandermonde identity shows

$$
\left(\begin{array}{c}
a X+b \\
c
\end{array}\right)=\sum_{w_{0}+w_{1}+\cdots+w_{a}=c, w_{0}, \ldots, w_{a} \in \mathbb{N}}\left(\begin{array}{c}
b \\
w_{0}
\end{array}\right) \prod_{v=1}^{a}\left(\begin{array}{c}
X \\
w_{v}
\end{array}\right),
$$

and then the statement follows from Lemma 4.9.

Lemma 4.9. Let $a, b$ be any nonnegative integers. Then there are nonnegative integers $e_{0}, \ldots, e_{a+b}$ such that

$$
\left(\begin{array}{c}
X \\
a
\end{array}\right)\left(\begin{array}{c}
X \\
b
\end{array}\right)=\sum_{i=0}^{a+b} e_{i}\left(\begin{array}{c}
X \\
i
\end{array}\right) \text { for all nonnegative integers } X .
$$

Proof. There are many proofs. This proof is due to Qiaochu Yuan and Brendan McKay. It is enough to prove the result for large enough integers $X$, because both sides can be regarded as polynomials in $X$. Now $\left(\begin{array}{c}X \\ a\end{array}\right)\left(\begin{array}{c}X \\ b\end{array}\right)$ is the number of ways to choose a subset of size $a$ and a subset of size $b$ from a set of size $X$. The union of these two subsets is a subset of size anywhere from $\max (a, b)$ to $a+b$, so $e_{i}$ is the number of different ways a subset of size $i$ can be realized as the union of a subset of size $a$ and a subset of size $b$.

This completes the proof of Lemma 4.4.

Lemma 4.10. $\mathcal{P}_{\theta, 3}=0$.

Proof. In trying to keep the notation simple, we give a detailed proof for $j=i+1$. The case $j>i+1$ uses the same argument. 
Let

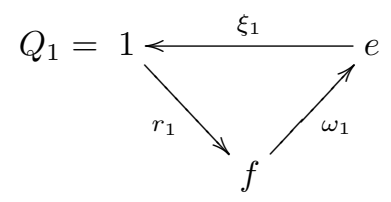

be the quiver at the seed $\mu_{1}\left(t_{i+1}\right)$, where $r_{1}, \omega_{1}$ and $\xi_{1}$ are the numbers of arrows. For a compatible pair $\beta=\left(S_{1}, S_{2}\right)$, let $|\beta|_{2}$ denote $\left|\mathcal{D}_{1}\right|-\left|S_{1}\right|$ and $|\beta|_{1}$ denote $\left|S_{2}\right|$.

Applying Theorem 3.21 to $x_{1 ; t_{i}}^{p} x_{f ; t_{i}}^{q}$, we obtain

$$
\begin{aligned}
& \sum_{\tau_{0 ; 1}, \ldots, \tau_{n_{1}-3 ; 1}}\left(\prod_{w=0}^{n_{1}-3}\left[\begin{array}{c}
A_{w+1 ; 1}-r_{1} s_{w ; 1} \\
\tau_{w ; 1}
\end{array}\right]\right) \\
& A_{n_{1}-1 ; 1}-r_{1} s_{n_{1}-2 ; 1} \geq 0 \\
& \times \widetilde{x_{1 ; t_{i+1}}} A_{n_{1}-1 ; 1}-r_{1} s_{n_{1}-2 ; 1} x_{f ; t_{i+1}}^{r_{1} s_{n_{1}-3 ; 1}-A_{n_{1}-2 ; 1}} x_{e ; t_{i+1}}^{\omega_{1} s_{n_{1}-2 ; 1}-\xi_{1} s_{n_{1}-3 ; 1}}
\end{aligned}
$$

$$
+x_{1 ; t_{i+1}}^{-A_{n_{1}-1 ; 1}} x_{f ; t_{i+1}}^{-A_{n_{1}-2 ; 1}} \sum_{\beta: r_{1}|\beta|_{1}-A_{n_{1}-1 ; 1}>0} x_{1 ; t_{i+1}}^{r_{1}|\beta|_{1}} x_{f ; t_{i+1}}^{r_{1}\left(A_{n_{1}-1 ; 1}-|\beta|_{2}\right)} x_{e ; t_{i+1}}^{\xi_{1}|\beta|_{2}-\left(\xi_{1} r_{1}-\omega_{1}\right)|\beta|_{1}},
$$

where the second sum is over all $\beta=\left(S_{1}=\bigcup_{i=1}^{p+q} S_{1}^{i}, S_{2}=\bigcup_{i=1}^{p+q} S_{2}^{i}\right)$ such that

$$
\left(S_{1}^{i}, S_{2}^{i}\right) \text { is a compatible pair in } \begin{cases}\mathcal{D}^{c_{n_{1}-1 ; 1} \times c_{n_{1}-2 ; 1}} & \text { if } 1 \leq i \leq q, \\ \mathcal{D}^{c_{n_{1} ; 1} \times c_{n_{1}-1 ; 1}} & \text { if } q+1 \leq i \leq p+q .\end{cases}
$$

Let $n_{2}$ be the number of seeds between $\mu_{1}\left(t_{i+1}\right)$ and $t_{i+2}$ inclusive. Suppose that $n_{2}$ is an even integer. The case of $n_{2}$ odd is similar, except that the roles of $x_{1 ; t_{i}+1}$ and $x_{e ; t+1}$ are interchanged. Let

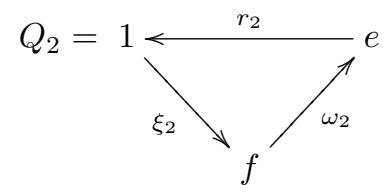

be the quiver at the seed $\mu_{1}\left(t_{i+2}\right)$, where $r_{2}, \omega_{2}$ and $\xi_{2}$ are the numbers of arrows.

Here we show that if $C_{2 ; i \rightarrow j} \bar{C}_{3 ; j \rightarrow j+1} \neq 0$ then $u_{2}+q_{3}^{(2)}$ (the exponent of $x_{f}$ ) can never be negative, so that the second sum in $\mathcal{P}_{\theta, 3}$ is equal to 0 . A similar argument can be applied to show that the other sums are 0 .

Let $p_{2}=A_{n_{1}-1 ; 1}-r_{1} s_{n_{1}-2 ; 1}$ and $q_{2}=\omega_{1} s_{n_{1}-2 ; 1}-\xi_{1} s_{n_{1}-3 ; 1}$ be the exponents of $\widetilde{x_{1 ; t_{i+1}}}$ and $x_{e ; t_{i+1}}$ in (52), respectively. Applying Theorem 3.13 to $\widetilde{x_{1 ; t_{i+1}}} p_{2} x_{e ; t_{i+1}}^{q_{2}}$ in (52), we have 


$$
\begin{aligned}
\sum_{\substack{\tau_{0 ; 1}, \ldots, \tau_{n_{1}-3 ; 1} \\
A_{n_{1}-1 ; 1}-r_{1} s_{n_{1}-2 ; 1} \geq 0}}\left(\prod_{w=0}^{n_{1}-3}\left[\begin{array}{c}
A_{w+1 ; 1}-r_{1} s_{w ; 1} \\
\tau_{w ; 1}
\end{array}\right]\right) x_{f ; t_{i+1}}^{r_{1} s_{n_{1}-3 ; 1}-A_{n_{1}-2 ; 1}} \\
\quad \times \sum_{\beta} x_{1 ; t_{i+2}}^{r_{2}|\beta|_{1}-A_{n_{2}-1 ; 2}} x_{e ; t_{i+2}}^{A_{n_{2} ; 2}-r_{2}|\beta|_{2}} x_{f ; t_{i+2}}^{\xi_{2}|\beta|_{2}-\left(\xi_{2} r_{2}-\omega_{2}\right)|\beta|_{1}} \\
=\sum_{\substack{\tau_{0 ; 1}, \ldots, \tau_{n_{1}-3 ; 1} \\
A_{n_{1}-1 ; 1}-r_{1} s_{n_{1}-2 ; 1} \geq 0}}\left(\prod_{w=0}^{n_{1}-3}\left[\begin{array}{c}
A_{w+1 ; 1}-r_{1} s_{w ; 1} \\
\tau_{w ; 1}
\end{array}\right]\right) \\
\times \sum_{\beta} x_{1 ; t_{i+2}}^{r_{2}|\beta|_{1}-A_{n_{2}-1 ; 2}} x_{e ; t_{i+2}}^{A_{n_{2} ; 2}-r_{2}|\beta|_{2}} x_{f ; t_{i+2}}^{\xi_{2}|\beta|_{2}-\left(\xi_{2} r_{2}-\omega_{2}\right)|\beta|_{1}+r_{1} s_{n_{1}-3 ; 1}-A_{n_{1}-2 ; 1}}
\end{aligned}
$$

where each second sum is over all $\beta=\left(S_{1}=\bigcup_{i=1}^{p_{2}+q_{2}} S_{1}^{i}, S_{2}=\bigcup_{i=1}^{p_{2}+q_{2}} S_{2}^{i}\right)$ such that

$$
\left(S_{1}^{i}, S_{2}^{i}\right) \text { is a compatible pair in } \begin{cases}\mathcal{D}^{c_{n_{2}-1 ; 2} \times c_{n_{2}-2 ; 2}} & \text { if } 1 \leq i \leq q_{2} \\ \mathcal{D}^{c_{n_{2} ; 2} \times c_{n_{2}-1 ; 2}} & \text { if } q_{2}+1 \leq i \leq p_{2}+q_{2}\end{cases}
$$

The exponent of $x_{1}$ is positive by definition of $\mathcal{P}_{\theta, 3}$. Therefore $A_{n_{2}-1 ; 2}<$ $r_{2}|\beta|_{1}$, hence $A_{n_{2}-1 ; 2} /\left(r_{2}|\beta|_{1}\right)<1$ and thus

$$
\frac{r_{1} A_{n_{2}-1 ; 2}}{c_{n_{2}-1}^{\left[r_{2}\right]} r_{2}}=\frac{r_{1}|\beta|_{1}}{c_{n_{2}-1}^{\left[r_{2}\right]}} \frac{A_{n_{2}-1 ; 2}}{r_{2}|\beta|_{1}}<\frac{r_{1}|\beta|_{1}}{c_{n_{2}-1}^{\left[r_{2}\right]}} \leq \xi_{2}|\beta|_{2}-\left(\xi_{2} r_{2}-\omega_{2}\right)|\beta|_{1},
$$

where the last inequality is proved in [21, Lemma 4.10] and [19, Proposition 4.1]. Using $r_{2}=\xi_{1}$ and the definition of $A_{n_{2}-1 ; 2}$, we get

$$
\begin{aligned}
& r_{1} s_{n_{1}-3 ; 1}-A_{n_{1}-2 ; 1}+\xi_{2}|\beta|_{2}-\left(\xi_{2} r_{2}-\omega_{2}\right)|\beta|_{1} \\
& \geq r_{1} s_{n_{1}-3 ; 1}-A_{n_{1}-2 ; 1} \\
&+\frac{r_{1}\left(c_{n_{2}}^{\left[\xi_{1}\right]}\left(A_{n_{1}-1 ; 1}-r_{1} s_{n_{1}-2 ; 1}\right)+c_{n_{2}-1}^{\left[\xi_{1}\right]}\left(\omega_{1} s_{n_{1}-2 ; 1}-\xi_{1} s_{n_{1}-3 ; 1}\right)\right)}{c_{n_{2}-1}^{\left[\xi_{1}\right]} \xi_{1}} \\
&=-A_{n_{1}-2 ; 1}+\frac{r_{1}\left(c_{n_{2}}^{\left[\xi_{1}\right]}\left(A_{n_{1}-1 ; 1}-r_{1} s_{n_{1}-2 ; 1}\right)+c_{n_{2}-1}^{\left[\xi_{1}\right]} \omega_{1} s_{n_{1}-2 ; 1}\right)}{c_{n_{2}-1}^{\left[\xi_{1}\right]} \xi_{1}} \\
&=-A_{n_{1}-2 ; 1}+\frac{r_{1}\left(c_{n_{2}}^{\left[\xi_{1}\right]} A_{n_{1}-1 ; 1}-\left(c_{n_{2}}^{\left[\xi_{1}\right]} r_{1}-c_{n_{2}-1}^{\left[\xi_{1}\right]} \omega_{1}\right) s_{n_{1}-2 ; 1}\right)}{c_{n_{2}-1}^{\left[\xi_{1}\right]} \xi_{1}} \\
&\left(A_{n_{1}-1 ; 1}-r_{1} s_{n_{1}-2 ; 1} \geq 0\right) c_{n_{2}-1}^{\left[\xi_{1}\right]} \xi_{1} \\
& \geq\left.\frac{r_{1}\left(c_{n_{2}}^{\left[\xi_{1}\right]} A_{n_{1}-1 ; 1}-\left(c_{n_{1}}^{\left[\xi_{1}\right]} r_{1}-1\right.\right.}{\geq}\left(\omega_{n_{2}-1}^{\left[\xi_{1}\right]} \omega_{1}\right) \frac{A_{n_{1}-1 ; 1}}{r_{1}}\right) \\
&\left.\xi_{1} A_{n_{1}-1 ; 1}-\xi_{1} A_{n_{1}-2 ; 1}\right) \stackrel{(51)}{>} 0 .
\end{aligned}
$$


Thus the exponent of $x_{f ; t_{i+2}}$ in the expansion of (52) is positive. The proof for the expansion of (53) uses a similar argument.

\section{§5. Example}

Example 5.1. Let $\Sigma_{t_{0}}$ be a seed connected to the initial seed $\Sigma_{t_{3}}$ by the following sequence of mutations:

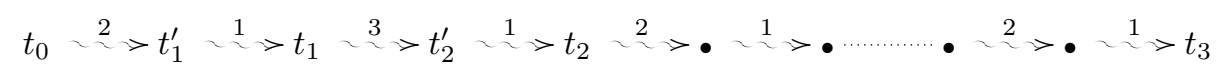

Suppose that the quivers corresponding to the first 5 seeds are as follows
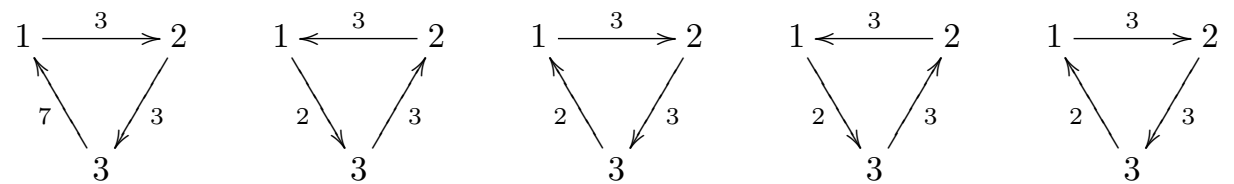

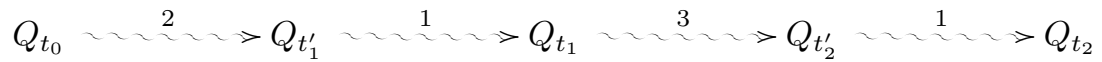

We want to illustrate the proof of positivity for $x_{2 ; t_{0}}$.

First, we compute its expansion in the cluster $\mathbf{x}_{t_{1}}$ using Theorem 3.21. The mutation sequence from $t_{0}$ to $t_{1}$ is at the vertices 1 and 2 . Thus we have $r=3$, $n=3, p=1, q=0$. Moreover,

$$
c_{1}=0, c_{2}=1, c_{3}=3, \quad A_{1}=1, A_{2}=3, \quad s_{1}=\tau_{0},
$$

and the summation is on $\tau_{0}=0,1$. The condition $s_{n-2} \leq A_{n-1} / r$ in the first sum of Theorem 3.21 becomes $\tau_{0} \leq 1$, which is always satisfied, so the second sum in the theorem is empty. Finally, the variables in the theorem are $x_{2}=x_{2 ; t_{1}}$, $z_{3}=x_{3 ; t_{1}}$ and $x_{3}=x_{1 ; t_{1}^{\prime}}=\left(x_{3 ; t_{1}}^{2}+x_{2 ; t_{1}}^{3}\right) / x_{1 ; t_{1}}$. Thus $x_{2 ; t_{0}}$ is equal to

$$
\begin{aligned}
x_{2 ; t_{1}}^{-1}\left(\begin{array}{l}
1 \\
0
\end{array}\right)\left(\frac{x_{3 ; t_{1}}^{2}+x_{2 ; t_{1}}^{3}}{x_{1 ; t_{1}}}\right)^{3}= & x_{2 ; t_{1}}^{-1}\left(\begin{array}{l}
1 \\
0
\end{array}\right) x_{1 ; t_{1}^{\prime}}^{3} \\
& +x_{2 ; t_{1}}^{-1}\left(\begin{array}{l}
1 \\
1
\end{array}\right) x_{3 ; t_{1}}^{3} .
\end{aligned}
$$

Now we compute the expansion of this expression in the cluster $\mathbf{x}_{t_{2}}$ again using Theorem 3.21. We treat the two terms (54) and (55) separately. For the first term, we need to expand $x_{1 ; t_{1}^{\prime}}^{3}$ which lies in the cluster $\mathbf{x}_{t_{1}^{\prime}}$. The mutation sequence from $t_{1}^{\prime}$ to $t_{2}$ is at the vertices 1 and 3 . Thus we have $r=2, n=4, p=3, q=0$. 
Moreover,

$$
\begin{array}{cl}
c_{1}=0, c_{2}=1, c_{3}=2, c_{4}=3, & A_{1}=3, A_{2}=6, A_{3}=9, \\
s_{1}=\tau_{0}, \quad s_{2}=2 \tau_{0}+\tau_{1}, & s_{3}=3 \tau_{0}+2 \tau_{1}+\tau_{2} .
\end{array}
$$

The two binomial coefficients in the first sum are $\left(\begin{array}{c}3 \\ \tau_{0}\end{array}\right)$ and $\left(\begin{array}{c}6-2 \tau_{0} \\ \tau_{1}\end{array}\right)$, so their product is zero unless

$$
0 \leq \tau_{0} \leq 3, \quad 0 \leq \tau_{1} \leq 6-2 \tau_{0} .
$$

Finally, the condition $s_{n-2} \leq A_{n-1} / r$ in the first sum of Theorem 3.21 implies that $\tau_{0}<3$, and that $\tau_{1} \leq 4$ if $\tau_{0}=0, \tau_{1} \leq 2$ if $\tau_{0}=1$, and $\tau_{1}=0$ if $\tau_{0}=2$. Therefore, the first sum is over the following pairs $\left(\tau_{0}, \tau_{1}\right)$

$$
(0,0)(0,1)(0,2)(0,3)(0,4)(1,0)(1,1)(1,2)(2,0)
$$

and the corresponding terms are

$$
\begin{aligned}
& x_{2 ; t_{2}}^{-1}\left(\begin{array}{l}
1 \\
0
\end{array}\right) x_{3 ; t_{2}}^{-6}\left(\begin{array}{l}
3 \\
0
\end{array}\right)\left(\begin{array}{l}
6 \\
0
\end{array}\right) x_{1 ; t_{2}^{\prime}}^{9} \\
& +x_{2 ; t_{2}}^{-1}\left(\begin{array}{l}
1 \\
0
\end{array}\right) x_{3 ; t_{2}}^{-6}\left(\begin{array}{l}
3 \\
0
\end{array}\right)\left(\begin{array}{l}
6 \\
1
\end{array}\right) x_{1 ; t_{2}}^{7} x_{2 ; t_{2}}^{3} \\
& +x_{2 ; t_{2}}^{-1}\left(\begin{array}{l}
1 \\
0
\end{array}\right) x_{3 ; t_{2}}^{-6}\left(\begin{array}{l}
3 \\
0
\end{array}\right)\left(\begin{array}{l}
6 \\
2
\end{array}\right) x_{1 ; t_{2}}^{5} x_{2 ; t_{2}}^{6} \\
& +x_{2 ; t_{2}}^{-1}\left(\begin{array}{l}
1 \\
0
\end{array}\right) x_{3 ; t_{2}}^{-6}\left(\begin{array}{l}
3 \\
0
\end{array}\right)\left(\begin{array}{l}
6 \\
3
\end{array}\right) x_{1 ; t_{2}}^{3} x_{2 ; t_{2}}^{9} \\
& +x_{2 ; t_{2}}^{-1}\left(\begin{array}{l}
1 \\
0
\end{array}\right) x_{3 ; t_{2}}^{-6}\left(\begin{array}{l}
3 \\
0
\end{array}\right)\left(\begin{array}{l}
6 \\
4
\end{array}\right) x_{1 ; t_{2}^{\prime}}^{1} x_{2 ; t_{2}}^{12} \\
& +x_{2 ; t_{2}}^{-1}\left(\begin{array}{l}
1 \\
0
\end{array}\right) x_{3 ; t_{2}}^{-4}\left(\begin{array}{l}
3 \\
1
\end{array}\right)\left(\begin{array}{l}
4 \\
0
\end{array}\right) x_{1 ; t_{2}^{\prime}}^{5} x_{2 ; t_{2}}^{3} \\
& +x_{2 ; t_{2}}^{-1}\left(\begin{array}{l}
1 \\
0
\end{array}\right) x_{3 ; t_{2}}^{-4}\left(\begin{array}{l}
3 \\
1
\end{array}\right)\left(\begin{array}{l}
4 \\
1
\end{array}\right) x_{1 ; t_{2}^{\prime}}^{3} x_{2 ; t_{2}}^{6} \\
& +x_{2 ; t_{2}}^{-1}\left(\begin{array}{l}
1 \\
0
\end{array}\right) x_{3 ; t_{2}}^{-4}\left(\begin{array}{l}
3 \\
1
\end{array}\right)\left(\begin{array}{l}
4 \\
2
\end{array}\right) x_{1 ; t_{2}^{\prime}}^{1} x_{2 ; t_{2}}^{9} \\
& +x_{2 ; t_{2}}^{-1}\left(\begin{array}{l}
1 \\
0
\end{array}\right) x_{3 ; t_{2}}^{-2}\left(\begin{array}{l}
3 \\
2
\end{array}\right)\left(\begin{array}{l}
2 \\
0
\end{array}\right) x_{1 ; t_{2}^{\prime}}^{1} x_{2 ; t_{2}}^{6} .
\end{aligned}
$$

The second sum in Theorem 3.21 is over all compatible pairs $\left(S_{1}, S_{2}\right)$ in $\mathcal{D}^{9 \times 6}$ such that $\left|S_{2}\right|>9 / 2$. The condition $\left|S_{2}\right|>9 / 2$ implies that $S_{2}$ must be equal to $\mathcal{D}_{2}$ or $\mathcal{D}_{2} \backslash\{$ any single vertical edge $\}$. If $S_{2}=\mathcal{D}_{2}$ then $S_{1}$ must be the empty set. If $S_{2}=\mathcal{D}_{2} \backslash\left\{v_{2 i-1}\right\}$ for $i=1,2,3$, then $S_{1}=\left\{u_{3 i-2}\right\}$ or $\emptyset$. If $S_{2}=\mathcal{D}_{2} \backslash\left\{v_{2 i}\right\}$ for $i=1,2,3$, then $S_{1}=\left\{u_{3 i}\right\}$ or $\emptyset$. Therefore the second sum in Theorem 3.21 is 
equal to

$$
\begin{aligned}
& x_{2 ; t_{2}}^{-1}\left(\begin{array}{l}
1 \\
0
\end{array}\right) 6 x_{3 ; t_{2}}^{-6} x_{1 ; t_{2}} x_{2 ; t_{2}}^{12} \\
& +x_{2 ; t_{2}}^{-1}\left(\begin{array}{l}
1 \\
0
\end{array}\right) x_{3 ; t_{2}}^{-6} x_{1 ; t_{2}}^{3} x_{2 ; t_{2}}^{9} \\
& +x_{2 ; t_{2}}^{-1}\left(\begin{array}{l}
1 \\
0
\end{array}\right) 6 x_{3 ; t_{2}}^{-4} x_{1 ; t_{2}} x_{2 ; t_{2}}^{9} .
\end{aligned}
$$

This shows that (54) is equal to the sum of all terms (56)-(67).

Applying Theorem 3.21 to the expression (55) and using a similar analysis, we see that $(55)$ is equal to

$$
\begin{aligned}
& x_{2 ; t_{2}}^{-1}\left(\begin{array}{l}
1 \\
1
\end{array}\right) x_{3 ; t_{2}}^{-3}\left(\begin{array}{l}
3 \\
0
\end{array}\right) x_{1 ; t_{2}^{\prime}}^{6} \\
& +x_{2 ; t_{2}}^{-1}\left(\begin{array}{l}
1 \\
1
\end{array}\right) x_{3 ; t_{2}}^{-3}\left(\begin{array}{l}
3 \\
1
\end{array}\right) x_{1 ; t_{2}^{\prime}}^{4} x_{2 ; t_{2}}^{3} \\
& +x_{2 ; t_{2}}^{-1}\left(\begin{array}{l}
1 \\
1
\end{array}\right) x_{3 ; t_{2}}^{-3}\left(\begin{array}{l}
3 \\
2
\end{array}\right) x_{1 ; t_{2}}^{2} x_{2 ; t_{2}}^{6} \\
& +x_{2 ; t_{2}}^{-1}\left(\begin{array}{l}
1 \\
1
\end{array}\right) x_{3 ; t_{2}}^{-3}\left(\begin{array}{l}
3 \\
3
\end{array}\right) x_{2 ; t_{2}}^{9} .
\end{aligned}
$$

So $x_{2 ; t_{0}}$ is equal to the sum of all terms (56)-(71). Observe that the powers of the variables $x_{1 ; t_{2}^{\prime}}, x_{1 ; t_{2}}$ in all terms are positive and that the powers of the variable $x_{2 ; t_{2}}$ are positive in all terms except for (56) and (68).

On the other hand,

$$
(56)+(68)=x_{3 ; t_{2}}^{-6} x_{1 ; t_{2}^{\prime}}^{6}\left(\frac{x_{1 ; t_{2}^{\prime}}^{3}+x_{3 ; t_{2}}^{3}}{x_{2 ; t_{2}}}\right)=x_{3 ; t_{2}}^{-6} x_{1 ; t_{2}^{\prime}}^{6} x_{2 ; t_{2}^{\prime \prime}}
$$

where $\mathbf{x}_{t_{2}^{\prime \prime}}=\mu_{2}\left(\mathbf{x}_{t_{2}^{\prime}}\right)$ denotes the cluster obtained from $\mathbf{x}_{t_{2}^{\prime}}$ by mutation at 2 .

Thus we obtain an expression for $x_{2 ; t_{0}}$ as a Laurent polynomial in the variables $x_{2 ; t_{2}^{\prime \prime}}, x_{1 ; t_{2}^{\prime}}, x_{1 ; t_{2}}, x_{2 ; t_{2}}, x_{3 ; t_{2}}$ with nonnegative coefficients and in which only the variable $x_{3 ; t_{2}}$ appears with negative powers. Note that $x_{2 ; t_{2}^{\prime \prime}}=\widetilde{\widetilde{x_{2 ; t_{2}}}}$ and $x_{1 ; t_{2}^{\prime}}=$ $\widetilde{x_{1 ; t_{2}}}$, thus the sum $(56)+(68)$ is of the form of the first sum in Theorem 4.3 , the sum of (57)-(64) and (69)-(71) is of the form of the second sum, and the sum of (65)-(67) is of the form of the third sum in Theorem 4.3.

Since the mutation sequence linking the seeds $\Sigma_{t_{2}^{\prime \prime}}, \Sigma_{t_{2}^{\prime}}$ and $\Sigma_{t_{2}}$ to the seed $\Sigma_{t_{3}}$ consists of mutations at the vertices 1 and 2 only, we see that $x_{3 ; t_{2}}=x_{3 ; t_{3}}$ and replacing the other variables with their expansions in the seed $\Sigma_{t_{3}}$ (which have nonnegative coefficients by the rank 2 case) produces again a Laurent polynomial with nonnegative coefficients in the cluster $\mathbf{x}_{t_{3}}$. 


\section{Acknowledgements}

We are grateful to Andrei Zelevinksy and anonymous referees for their valuable suggestions. Shortly after the original version of this paper [21] became available, a new parametrization of our rank 2 formula has been given in [19]. Following the referee's suggestion, we use this new parametrization here to present our result in this final version.

The first author is partially supported by the NSF grant DMS-0901367.

The second author is partially supported by the NSF grant DMS-1001637.

\section{References}

[1] I. Assem, G. Dupont, R. Schiffler and D. Smith, Friezes, strings and cluster variables, Glasgow Math. J. 54 (2012), 27-60. Zbl pre06005961 MR 2862382

[2] I. Assem, C. Reutenauer and D. Smith, Friezes, Adv. Math. 225 (2010), 3134-3165. Zbl pre05817898 MR 2729004

[3] A. Beineke, T. Brüstle and L. Hille, Cluster-cyclic quivers with three vertices and the Markov equation, Algebr. Represent. Theory 14 (2011), 97-112. Z Zbl 1239.16017 MR 2763295

[4] A. Berenstein, S. Fomin and A. Zelevinsky, Cluster algebras III: Upper bounds and double Bruhat cells, Duke Math. J. 126 (2005), 1-52. Zbl 1135.16013 MR 2110627

[5] A. Berenstein and A. Zelevinsky, Quantum cluster algebras, Adv. Math. 195 (2005), 405455. Zbl 1124.20028 MR 2146350

[6] P. Caldero and F. Chapoton, Cluster algebras as Hall algebras of quiver representations, Comment. Math. Helv. 81 (2006), 595-616. Zbl 1119.16013 MR 2250855

[7] P. Caldero and B. Keller, From triangulated categories to cluster algebras, Invent. Math. 172 (2008), 169-211. Zbl 1141.18012 MR 2385670

[8] H. Derksen, J. Weyman and A. Zelevinsky, Quivers with potentials and their representations II: applications to cluster algebras, J. Amer. Math. Soc. 23 (2010), 749-790. Zbl 1208.16017 MR 2629987

[9] A. I. Efimov, Quantum cluster variables via vanishing cycles, arXiv:1112.3601.

[10] A. Felikson, M. Shapiro and P. Tumarkin, Cluster algebras and triangulated orbifolds, Adv. Math. 231 (2012), 2953-3002. Zbl 1256.13014 MR 2970470

[11] V. Fock and A. Goncharov, Moduli spaces of local systems and higher Teichmüller theory, Publ. Math. Inst. Hautes Études Sci. 103 (2006), 1-211. Zbl 1099.14025 MR 2233852

[12] S. Fomin, M. Shapiro, and D. Thurston, Cluster algebras and triangulated surfaces. Part I: Cluster complexes, Acta Math. 201 (2008), 83-146. Zbl pre05508850 MR 2448067

[13] S. Fomin and A. Zelevinsky, Cluster algebras I: Foundations, J. Amer. Math. Soc. 15 (2002), 497-529. Zbl 1021.16017 MR 1887642

[14] S. Fomin and A. Zelevinsky, Cluster algebras IV: Coefficients, Compos. Math. 143 (2007), 112-164. Zbl 1127.16023 MR 2295199

[15] C. Fu and B. Keller, On cluster algebras with coefficients and 2-Calabi-Yau categories, Trans. Amer. Math. Soc. 362 (2010), 859-895. Zbl 1201.18007 MR 2551509

[16] D. Hernandez and B. Leclerc, Cluster algebras and quantum affine algebras, Duke Math. J. 154 (2010), 265-341. Zbl pre05788166 MR 2682185 
[17] Y. Kimura and F. Qin, Graded quiver varieties, quantum cluster algebras and dual canonical basis, arXiv:1205.2066.

[18] K. Lee, On cluster variables of rank two acyclic cluster algebras, Ann. Combin. 16 (2012), 305-317. Zbl pre06121624 MR 2927610

[19] K. Lee, L. Li and A. Zelevinsky, Greedy elements in rank 2 cluster algebras, Selecta Math. (N.S.), to appear.

[20] K. Lee and R. Schiffler, A combinatorial formula for rank 2 cluster variables, J. Algebr. Combin. 37 (2013), 67-85. Zbl pre06138612 MR 3016302

[21] K. Lee and R. Schiffler, Positivity for cluster algebras of rank 3 (version 1), arXiv:1205.5466v1.

[22] G. Musiker, R. Schiffler and L. Williams, Positivity for cluster algebras from surfaces, Adv. Math. 227 (2011), 2241-2308. Zbl pre05919159 MR 2807089

[23] H. Nakajima, Quiver varieties and cluster algebras, Kyoto J. Math. 51 (2011), 71-126. Zbl 1223.13013 MR 2784748

[24] F. Qin, Quantum cluster variables via Serre polynomials, J. Reine Angew. Math. 668 (2012), 149-190. Zbl 1252.13013 MR 2948875

[25] D. Rupel, Proof of the Kontsevich non-commutative cluster positivity conjecture, C. R. Math. Acad. Sci. Paris 350 (2012), 929-932. Zbl pre06115445 MR 2996767

[26] R. Schiffler, On cluster algebras arising from unpunctured surfaces. II, Adv. Math. 223 (2010), 1885-1923. Zbl 1238.13029 MR 2601004

[27] R. Schiffler, A cluster expansion formula ( $A_{n}$ case). Electron. J. Combin. 15 (2008), no. 1, res. paper 64, 9 pp. Zbl 1184.13064 MR 2398856

[28] R. Schiffler and H. Thomas, On cluster algebras arising from unpunctured surfaces, Int. Math. Res. Notices 2009, no. 17, 3160-3189. Z Zbl 1171.30019 MR 2534994 